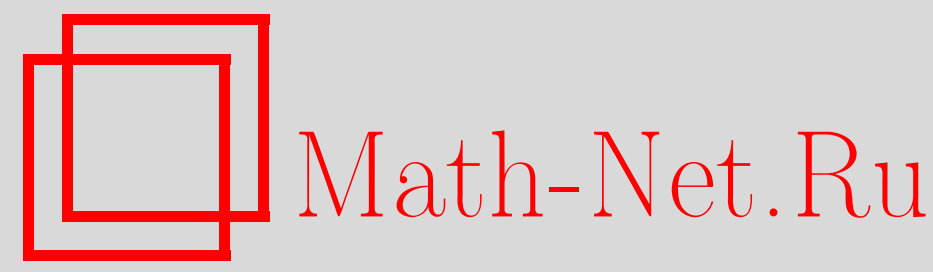

Ю. Н. Дрожжинов, Б. И. Завьялов, Теоремы тауберова типа для обобщенной мультипликативной свертки, Изв. РАН. Сер. матем., 2000, том 64, выпуск 1, 37-94

DOI: https://doi.org/10.4213/im274

Использование Общероссийского математического портала Math-Net.Ru подразумевает, что вы прочитали и согласны с пользовательским соглашением

http://www . mathnet.ru/rus/agreement

Параметры загрузки:

IP: 54.166 .219 .16

26 апреля 2023 г., $17: 13: 35$ 
УДК 517.5

Ю.Н. Дрожжинов, Б.И. Завьялов

\title{
Теоремы тауберова типа для обобщенной мультипликативной свертки
}

\begin{abstract}
Обсуждается следующая задача. Пусть $f$ - обобщенная функция медленного роста с носителем на положительной полуоси, а $\varphi_{k}$ - последовательность "пробных" функций такая, что $\varphi_{k} \rightarrow \varphi_{0}$ при $k \rightarrow+\infty$ в некотором функциональном пространстве. Пусть существует предел $\frac{1}{\rho(k)}\left(f(k t), \varphi_{k}(t)\right) \rightarrow c, k \rightarrow+\infty$, где $\rho(k)$ - правильно меняющаяся функция. Требуется найти условия, при которых существует предел $\frac{1}{\rho(k)}(f(k t), \varphi(t)) \rightarrow c_{\varphi}, k \rightarrow+\infty$, для всех основных функций $\varphi$. Сформулированы и доказаны теоремы, в которых приведены общие достаточные условия, решающие поставленную выше задачу. В качестве приложения изучается вопрос существования квазиасимптотики решения обыкновенного дифференциального уравнения с переменными коэффициентами. Доказаны абелевы и тауберовы теоремы для широкого класса интегральных преобразований обобщенных функций таких, например, как обобщенное интегральное преобразование Стилтьеса.

Библиография: 22 наименования
\end{abstract}

\section{Введение}

Известная тауберова теорема Н. Винера [5], [6] в ее мультипликативном варианте гласит. Пусть $f(t) \in L_{\infty}(0,+\infty)$, а функция $\varphi_{0}(t) \in L_{1}(0,+\infty)$ имеет преобразование Меллина

$$
\widehat{\varphi}_{0}(x) \equiv \int_{0}^{\infty} t^{-i x} \varphi_{0}(t) d t \neq 0 \quad \text { для всех } x \in(-\infty,+\infty) .
$$

Тогда из существования предела мультипликативной свертки $f(t)$ с $\varphi_{0}(t)$ :

$$
\lim _{k \rightarrow+\infty} \int_{0}^{\infty} f(k t) \varphi_{0}(t) d t=c,
$$

следует существование аналогичного предела для свертки $f(t)$ с любой $\varphi(t) \in$ $L_{1}(0,+\infty)$.

Эта теорема играет важную роль в гармоническом анализе и имеет многочисленные применения в различных областях математики (см. [7]-[12], [14]). Вместе с тем, с точки зрения ее применения в современной математической физике, условие принадлежности функций $f$ и $\varphi$ пространствам $L_{\infty}$ и $L_{1}$ является весьма ограничительным. С другой стороны, весьма часто в приложениях случается ситуация,

Работа выполнена при финансовой поддержке РФФИ (гранты № 99-01-00080 и № 9615-96131).

(C) Ю.Н. Дрожжинов, Б. И. ЗАвьялов, 2000 
когда вместо "чистой" мультипликативной свертки $\left(f(k t), \varphi_{0}(t)\right)$ приходится рассматривать "испорченную" (обобщенную) свертку

$$
F(k)=\left(f(k t), \varphi_{k}(t)\right) .
$$

Здесь семейство ${ }^{1}\left\{\varphi_{k}(t), k>k_{0}\right\}$ таково, что $\varphi_{k}(t) \rightarrow \varphi_{0}(t), k \rightarrow+\infty$.

В предыдущей статье авторов [1] доказан ряд теорем типа Винера для чистой мультипликативной свертки в классах обобщенных функций медленного роста в наиболее общей асимптотической шкале - шкале автомодельных (правильно меняюшихся) функций.

В данной работе мы изучаем обобщенную мультипликативную свертку. Задача состоит в следующем. Пусть $f \in \mathscr{S}_{+}^{\prime}$, т.е. является обобщенной функцией медленного роста с носителем на положительной полуоси, а $\varphi_{k}(t)$ - последовательность "пробных" функций такая, что $\varphi_{k} \rightarrow \varphi_{0}$ при $k \rightarrow+\infty$ в некотором функциональном пространстве (в каком именно уточняется далее). Пусть, кроме того, $\rho(k)-$ автомодельная функция и существует предел

$$
\frac{1}{\rho(k)}\left(f(k t), \varphi_{k}(t)\right) \longrightarrow c, \quad k \rightarrow+\infty .
$$

Требуется найти условия, при которых существует предел

$$
\frac{1}{\rho(k)}(f(k t), \varphi(t)) \longrightarrow c_{\varphi}, \quad k \rightarrow+\infty,
$$

для всех основных функций $\varphi(t)$. Представляет также интерес случай, когда предел (0.4) существует не на одной последовательности, а на семействе последовательностей $\varphi_{k}^{\beta}(t)$, где $\beta$ принадлежит некоторому абстрактному множеству. Это тем более интересно, что, как будет показано ниже, функции $\varphi_{k}(t)$ не могут принадлежать классическому основному пространству Шарца $\mathscr{S}$, в то время как второй случай это допускает. Это особенно важно при изучении асимптотических свойств широкого класса интегральных преобразований (см. ниже).

$\mathrm{B} \S 1$ вводятся необходимые определения и обозначения и напоминаются некоторые факты из теории обобщенных функций.

В $\S 2$ обсуждаются пространства основных функций $\mathscr{S}_{b, N, \delta}^{a, M}$, где $a, M$ характеризуют поведение и гладкость этих функций на бесконечности, а $b, N$ - в окрестности нуля. Эти пространства введены в работе [1] и являются важным элементом дальнейших рассмотрений. Мы приводим основные свойства этих пространств, преобразование Меллина функций из них, лемму о свертке и др. Эти свойства достаточно традиционны и в схожих ситуациях изучались, например, в работах $[2],[11],[12]$.

В $\S 3$ мы изучаем банаховы пространства семейства последовательностей основных функций, вводим обобщенную мультипликативную свертку и изучаем некоторые ее свойства.

В $\S 4$ рассматриваются квазиасимптотические свойства обобщенных функций из введенных пространств и доказываются основные леммы. $\S 5$ центральньй.

\footnotetext{
${ }^{1}$ В дальнейшем вместо слова "семейство" нам удобно употреблять слово “последовательность".
} 
Здесь сформулированы и доказаны основные теоремы, в которых приведены общие достаточные условия, решаюшие поставленные выше задачи.

$\S 6$ и 7 посвящены некоторым приложениям. В $\S 6$ мы изучаем вопрос сушествования квазиасимптотики решения обыкновенного дифференциального уравнения с переменными коэффициентами. Найдены достаточные условия на эти коэффициенты, обеспечиваюшие существование указанной квазиасимптотики, если правая часть обладает ею. Здесь же доказана теорема о некомпенсации особенностей голоморфиых функций. В $\S 7$ доказываются абелевы и тауберовы теоремы для интегральных преобразований обобщенных функций таких, как обобщенное интегральное преобразование Стилтьеса, интегральное преобразование с логарифмическим ядром и с ядром арктангенса. Излагаемый здесь метод применим и для широкого класса других интегральных преобразований.

Наконец, в приложении (§ 8) доказывается ряд вспомогательных утверждений, доказательство которых в основном тексте, на наш взгляд, сильно перегрузило бы изложение и затруднило восприятие основных результатов.

\section{§ 1. Основные определения и обозначения}

Пусть $a, b$ - нецелые вещественные числа, $a>b$. Обозначим через $\Pi_{b}^{a}$ полосу

$$
\Pi_{b}^{a}=\{z=x+i y \in \mathbb{C}: b<y<a, x \in \mathbb{R}\}
$$

и через $\bar{\Pi}_{b}^{a}-$ ее замыкание.

При нецелых $x<-1$ определим функцию $\langle x\rangle$ формулой

$$
\langle x\rangle=[-x-1],
$$

где $[\xi]$ - целая часть числа $\xi$. Выполнено неравенство

$$
-2<\langle x\rangle+x<-1 \text {. }
$$

Если функция $\varphi(t)$ имеет в нуле $m$ производных, то ее многочлен Тейлора в нуле порядка $m$ обозначается

$$
T_{\varphi(t)}^{m} \equiv T_{\varphi}^{m}=\sum_{j=0}^{m} \frac{\varphi^{(j)}(0)}{j !} t^{j}, \quad \varphi^{(j)}(0)=\left.\frac{d^{j}}{d t^{j}} \varphi(t)\right|_{t=0} .
$$

Положительная и непрерьвная при достаточно больших $k$ функция $\rho(k)$ называется автомодельной (правильно меняющейся), если существует предел

$$
\lim _{k \rightarrow+\infty} \frac{\rho(k t)}{\rho(k)}=\psi(t)
$$

причем сходимость равномерная по $t$ на каждом компакте полуоси $(0, \infty)$. Легко видеть, что $\psi(t)=t^{\alpha}$ при некотором $\alpha$. В этом случае функцию $\rho(k)$ называем автомодельной порядка $\alpha$ (см. [4]).

В дальнейшем автомодельные функции играют роль асимптотической шкалы, и нам понадобятся некоторые их свойства, которые мы сформулируем в виде леммы. 
Лемма 1. Пусть $\rho(k)$ - автомодельная функиия порядка $\alpha$. Тогда для любого $\varepsilon>0$ существует $k_{0}$ mакое, что:

$$
\begin{aligned}
t^{\alpha-\varepsilon} \leqslant \frac{\rho(k t)}{\rho(k)} \leqslant t^{\alpha+\varepsilon} \quad \text { npu } & k>k_{0}, \quad t>1+\varepsilon, \\
t^{\alpha+\varepsilon} \leqslant \frac{\rho(k t)}{\rho(k)} \leqslant t^{\alpha-\varepsilon} \quad \text { npu } & \frac{k_{0}}{k}<t<1-\varepsilon .
\end{aligned}
$$

Подробнее см. [3].

ЗАмечАниЕ. Пусть $\rho(k)$ - автомодельная функция порядка $\alpha$, a $\left\{\rho_{\beta}(k), \beta \in I\right\}-$ семейство положительных функций, определенных при $k>k_{1}$ (здесь $I$ - некоторое множество индексов). Пусть существует постоянная $c$, не зависящая от $\beta$, так что $\frac{\rho_{\beta}(k)}{\rho(k)}<c$ при $k>k_{1}$. Тогда для любого $\varepsilon>0$ существует постоянная $k_{2}$, не зависяшая от $\beta$, такая, что

$$
\frac{\rho_{\beta}(t k)}{\rho(k)} \leqslant\left\{\begin{array}{lll}
c t^{\alpha+\varepsilon} & \text { при } k>k_{2}, \quad t>1+\varepsilon, \\
c t^{\alpha-\varepsilon} & \text { при } \frac{k_{2}}{k}<t<1-\varepsilon .
\end{array}\right.
$$

Действительно, при данном $\varepsilon>0$ и $k_{0}$ таком, как в лемме, при $k>k_{2}=$ $\max \left\{k_{0}, k_{1}\right\}$ имеем

$$
\frac{\rho_{\beta}(k t)}{\rho(k)}=\frac{\rho_{\beta}(k t)}{\rho(k t)} \frac{\rho(k t)}{\rho(k)} \leqslant \begin{cases}c t^{\alpha+\varepsilon} \text { при } k_{1} \leqslant k_{2}<k, \quad t>1+\varepsilon \\ c t^{\alpha-\varepsilon} \text { при } \frac{k_{0}}{k} \leqslant \frac{k_{2}}{k}<t<1-\varepsilon .\end{cases}
$$

Стандартное пространство Шварца бесконечно дифференцируемых быстро убываюших функций обозначаем, как обычно, через $\mathscr{S}$, так что $\mathscr{S}^{\prime}$ - стандартное пространство обобщенных функций медленного роста. Пространство обобщенных функций медленного роста с носителями на $[0,+\infty)$ обозначается через $\mathscr{S}_{+}^{\prime}$, оно является сопряженным к пространству $\mathscr{S}_{+}$бесконечно дифференцируемых на $[0,+\infty)$ функций $\varphi$ таких, что

$$
\max _{|j| \leqslant m} \sup _{0 \leqslant t<+\infty}\left(1+t^{m}\right)\left|\varphi^{(j)}(t)\right|<\infty, \quad m=0,1, \ldots
$$

ОПРЕдЕЛЕНИЕ 1. Говорят, что обобщенная функция $f(t) \in \mathscr{S}_{+}^{\prime}$ обладает квазиасимптотикой относительно функиии $\rho(k)$ на функиии $\varphi(t)$, если сушествует

$$
\lim _{k \rightarrow+\infty} \frac{1}{\rho(k)}(f(k t), \varphi(t))=\text { const. }
$$

Если существует предел

$$
\lim _{k \rightarrow \infty} \frac{1}{\rho(k)}(f(k t), \varphi(t))=c_{\varphi} \text { для любой } \varphi(t) \in \mathscr{S}_{+},
$$

то мы говорим, что $f(t)$ обладает квазиасимптотикой относительно $\rho(k)$.

Если $c_{\varphi}=0$ для $\forall \varphi \in \mathscr{I}_{+}$, то будем говорить, что $f(t)$ обладает тривиальной квазиасимптотикой. 
Если

$$
\frac{1}{\rho(k)}(f(k t), \varphi(t))=O(1) \text { при } k \rightarrow+\infty \quad \text { для любой } \varphi(t) \in \mathscr{S}_{+},
$$

то говорят, что $f(t)$ квазиасимптотически ограничена относительно $\rho(k)$.

Отметим, что если $f(t)$ обладает квазиасимптотикой относительно некоторой $\rho(k)$, то $\rho(k)$ - обязательно автомодельная функция какого-то порядка $\alpha$, а $c_{\varphi}$ в формуле (1.5) равно const $\left(f_{\alpha+1}(t), \varphi(t)\right)$, где $f_{\beta}(t)$ - лиувиллевское ядро дробного интегрирования и дифференцирования:

$$
f_{\beta}(t)= \begin{cases}\frac{\Theta(t) t^{\beta-1}}{\Gamma(\beta)} & \text { при } \beta>0, \\ \frac{d^{N}}{d t^{N}} f_{\beta+N}(t) & \text { при } \beta \leqslant 0, \quad \beta+N>0 .\end{cases}
$$

Здесь $\Theta(t)$ - функция Хевисайда: $\Theta(t)=0$ при $t<0$ и $\Theta(t)=1$ при $t>0$.

Пусть $f(t) \in \mathscr{S}_{+}^{\prime}$. Обобщенная функция $f^{(-\beta)}(t)$, равная (аддитивной) свертке обобщенных функций $f$ и $f_{\beta}$, назьвается первообразной (при $\beta<0$ - производной) порядка $\beta$.

Обобшенная функция $f(t) \in \mathscr{S}_{+}^{\prime}$ тогда и только тогда обладает квазиасимптотикой относительно $\rho(k)$, когда существует $N$ такое, что $(N$-я первообразная $f(t))$ функция $f^{(-N)}(t)$ непрерывна и $f^{(-N)}(t) \sim c t^{N} \rho(t)$ при $t \rightarrow+\infty$, где $c$ - некоторая постоянная.

Аналогично определяется квазиасимптотика $f(t)$ в начале координат (подробнее см. [3]).

Преобразование Лапласа

$$
L[f] \equiv \tilde{f}(z)=\left(f(t), e^{i z t}\right), \quad f \in \mathscr{S}_{+}^{\prime},
$$

осушествляет изоморфизм пространства $\mathscr{S}_{+}^{\prime}$ на пространство $H\left(\mathbb{R}_{+}\right)$голоморфных в верхней полуплоскости функций, удовлетворяюших оценке полиномиального роста:

$$
|\tilde{f}(x+i y)| \leqslant M \frac{(1+|z|)^{c}}{y^{d}}, \quad y>0,
$$

при некоторых $M, c, d$ (см. [13]).

Нам понадобятся стандартные обобщенные функции

$$
t_{+}^{\lambda} \ln ^{m} t_{+} \in \mathscr{S}_{+}^{\prime}, \quad \lambda \in \mathbb{C}, \quad m=0,1,2, \ldots
$$

(см. [2]). Напомним, что при $\operatorname{Re} \lambda>-1$ они являются регулярными обобщенными функциями, совпадаюшими с $\Theta(t)(\ln t)^{m} t^{\lambda}$. Кроме того, они аналитичны по $\lambda$ во всей комплексной плоскости, кроме $\lambda=-1,-2, \ldots$, в которых они имеют полюса порядка $m+1$. Обобшенные функции $t_{+}^{-n} \ln ^{m} t_{+}, n=1,2, \ldots$, определяются как коэффициенты при $(\lambda+n)^{m}$ в лорановском разложении функции $t_{+}^{\lambda}$ в точке $\lambda=-n$.

Функции $t_{+}^{\lambda}$ при $\lambda \neq-1,-2, \ldots-$ однородные функции степени $\lambda$ :

$$
(k t)_{+}^{\lambda}=k^{\lambda} t_{+}^{\lambda}, \quad k>0 .
$$


Кроме того, при $\lambda \neq-1,-2, \ldots$ имеем $\frac{d^{m}}{d \lambda^{m}} t_{+}^{\lambda}=t_{+}^{\lambda} \ln ^{m} t_{+}$. Уравнение

$$
\left(t \frac{d}{d t}-\lambda\right)^{m} y=0, \quad y \in \mathscr{S}_{+}^{\prime}, \quad \lambda \neq-1,-2, \ldots,
$$

имеет $m$ линейно независимых решений

$$
t_{+}^{\lambda}, \quad t_{+}^{\lambda} \ln t_{+}, \quad \ldots, \quad t_{+}^{\lambda} \ln ^{m-1} t_{+} .
$$

При $\lambda=-n, n=1,2, \ldots$, уравнение

$$
\left(t \frac{d}{d t}+n\right)^{m} y=0, \quad y \in \mathscr{S}_{+}^{\prime}
$$

имеет $m$ линейно независимых решений

$$
\delta^{(n-1)}(t), \quad t_{+}^{-n}, \quad t_{+}^{-n} \ln t_{+}, \quad \ldots, \quad t_{+}^{-n} \ln ^{m-2} t_{+} .
$$

(Подробнее см. $[2, \S 4]$.

\section{§ 2. Пространства $\mathscr{S}_{b, N, \delta}^{a, M}$ основных и $\left(\mathscr{S}_{b, N, \delta}^{a, M}\right)^{\prime}$ обобщенных функций}

Пусть $M, N$ - целые неотрицательные числа, $a, b$ - вешественные нецелые числа и $\delta>0$. Пусть, далее, $\varphi(t)$ - гладкая функция, определенная на замкнутой полуоси $[0,+\infty)$. Положим

$$
Q_{b, N, \delta}[\varphi]=\max _{j \leqslant N} \int_{0}^{\delta} t^{b}\left|t^{j} \frac{d^{j}}{d t^{j}}\left\{\varphi(t)-T_{\varphi}^{\langle b\rangle}(t)\right\}\right| d t .
$$

Здесь, как и в $\S 1,\langle b\rangle=[-b-1]$ при $b<-1$. Если $b>-1$, то тейлоровский многочлен $T_{\varphi}^{\langle b\rangle}(t)$ в (2.1) отсутствует. Положим также

$$
V^{a, M, \delta}[\varphi]=\max _{j \leqslant M} \int_{\delta}^{+\infty} t^{a}\left|t^{j} \frac{d^{j}}{d t^{j}} \varphi(t)\right| d t .
$$

Введем норму

$$
\mathscr{P}_{b, N, \delta}^{a, M}[\varphi]=Q_{b, N, \delta}[\varphi]+V^{a, M, \delta}[\varphi]+\sum_{j=0}^{\langle b\rangle}\left|\varphi^{(j)}(0)\right|
$$

(если $b>-1$, то последняя сумма в (2.2) отсутствует).

Пополнение пространства бесконечно дифференцируемых на $[0,+\infty)$ и быстро убывающих вместе со всеми своими производными функций по этой норме обозначается $\mathscr{S}_{b, N, \delta}^{a, M}$. Функции $\varphi \in \mathscr{S}_{b, N, \delta}^{a, M}$ можно описать следуюшим образом:

$$
\varphi(t)=c_{0}+\frac{c_{1}}{1 !} t+\cdots+\frac{c_{\langle b\rangle}}{\langle b\rangle !} t^{\langle b\rangle}+\psi(t)
$$


где

$$
\begin{array}{ll}
t^{b}\left|t^{j} \psi^{(j)}(t)\right| \in L_{1}(0, \delta), & 0 \leqslant j \leqslant N, \\
t^{a}\left|t^{j} \varphi^{(j)}(t)\right| \in L_{1}(\delta \pm \varepsilon,+\infty), & 0 \leqslant j \leqslant M .
\end{array}
$$

Здесь $\varepsilon$ любое, $0<\varepsilon<\delta$, причем знак плюс во второй формуле (2.4) берется при $M>N$ и знак минус в случае $M \leqslant N$. В случае $b>-1$ полином в $(2.3)$ отсутствует.

Если $M=N$, то $\mathscr{S}_{b, N, \delta_{1}}^{a, M}=\mathscr{S}_{b, N, \delta_{2}}^{a, M}$ для любых $\delta_{1}$ и $\delta_{2}$, больших нуля. В этом случае индекс $\delta$ мы, как правило, опускаем.

Числа $c_{j}, \quad j=0,1, \ldots,\langle b\rangle$, определяются однозначно и являются естественным расширением производных в нуле функций из $\mathscr{S}_{b, N, \delta}^{a, M}$. Всюду далее мы их обозначаем как $\varphi^{(j)}(0)$, так что $c_{j}=\varphi^{(j)}(0), j=0,1, \ldots,\langle b\rangle$. Соответственно $T_{\varphi}^{\langle b\rangle}(t)=\sum_{j=0}^{\langle b\rangle} \frac{c_{j}}{j !} t^{j}$.

Пространство $\mathscr{S}_{b, N, \delta}^{a, M}$ можно также описать следующей эквивалентной нормой:

$$
\begin{aligned}
\underset{b, N, \delta}{\mathscr{P} * a, M}[\varphi]= & \max _{j \leqslant N} \int_{0}^{\delta} t^{b}\left|\left(t \frac{d}{d t}\right)^{j}\left\{\varphi(t)-T_{\varphi}^{\langle b\rangle}(t)\right\}\right| d t \\
& +\max _{j \leqslant M} \int_{\delta}^{\infty} t^{a}\left|\left(t \frac{d}{d t}\right)^{j} \varphi(t)\right| d t+\sum_{j=0}^{\langle b\rangle}\left|\varphi^{(j)}(0)\right| .
\end{aligned}
$$

Проективный предел по $M$ и $N$ пространств $\mathscr{S}_{b, N, \delta}^{a, M}$ обозначается как $\mathscr{S}_{b}^{a}$. Проективный предел по $a$ и $b$ этих пространств образует пространство $\mathscr{I}_{+}$, так что

$$
\mathscr{S}_{b}^{a}=\mathscr{S}_{b, \infty}^{a, \infty}=\bigcap_{M, N \in \mathscr{Z}_{+}} \mathscr{S}_{b, N, \delta}^{a, M}, \quad \mathscr{S}_{+}=\bigcap_{a, b \in \mathbb{R}} \mathscr{S}_{b}^{a} .
$$

В дальнейшем нам понадобятся следующие подпространства пространства $\mathscr{S}_{b, N, \delta}^{a, M}$

$$
\begin{aligned}
& \stackrel{\mathscr{S}}{a, M}_{b, N, \delta}^{a, M}=\left\{\varphi(t) \in \mathscr{S}_{b, N, \delta}^{a, M}: \varphi^{(j)}(0)=0, \quad j=0, \ldots,\langle a\rangle\right\}, \\
& \mathscr{S}_{b, N, \delta}^{a, M}=\left\{\varphi(t) \in \mathscr{S}_{b, N, \delta}^{a, M}: \varphi^{(j)}(0)=0, \quad j=0, \ldots,\langle b\rangle\right\} .
\end{aligned}
$$

Если $b>-1$, то $\mathscr{S}_{0}^{a, M} b$, совпадает с $\mathscr{S}_{b, N, \delta}^{a, M}$, если $a>-1$, то $\stackrel{0}{\mathscr{S}}_{b, N, \delta}^{a, M}$ совпадает с $\mathscr{S}_{b, N, \delta}^{a, M}$. В случае $a>b$ имеем $\mathscr{S}_{0}^{a, M} \underset{b, N, \delta}{ } \subset \stackrel{\mathscr{S}}{b, N, \delta}_{b, M}^{a,} \subset \mathscr{S}_{b, N, \delta}^{a, M}$. Если $\varphi(t) \in \mathscr{S}_{b, N, \delta}^{a, M}$, TO

$$
\varphi(t)-T_{\varphi}^{\langle a\rangle}(t) \equiv \stackrel{o}{\varphi}(t) \in \stackrel{0}{\mathscr{S}}_{b, N, \delta}^{a, M}
$$

Аналогично определяются пространства $\stackrel{0}{\mathscr{S}_{b}^{a}}$ и $_{0}^{\mathscr{S}} \underset{b}{a}$.

Положим еше

$$
{ }_{p} \mathscr{S}_{+}=\left\{\varphi(t) \in \mathscr{S}_{+}: \varphi^{(j)}(0)=0, \quad j=0,1, \ldots, p\right\}
$$


где $p$ - целое неотрищательное число. Отметим, что $\langle a\rangle \mathscr{S}_{+}$плотно в $\stackrel{0}{\mathscr{S}} \underset{b, N, \delta}{a, m}$, а пространство бесконечно дифференцируемых функций с компактными носителями в $(0,+\infty)$ плотно в $\underset{0}{\mathscr{S}} \underset{b, N, \delta}{a, M}$ при любых $a, b(b \leqslant a), M, N$ и $\delta$.

Сопряженные пространства (пространства линейных непрерывных функционалов) образуют соответствующие пространства обобщенных функций и обозначаются, как обычно, штрихом сверху. Так, например, $f \in\left(\mathscr{S}_{b, N, \delta}^{a, M}\right)^{\prime}$ означает, что $f-$ линейный непрерывный функционал над пространством основных функций $\mathscr{S}_{b, N, \delta}^{a, M}$.

Мы будем писать $f \in\left(\mathscr{S}^{a}, M\right)^{\prime}$, если при некотором $\eta>0$ функционал $f$ принадлежит $\left(\mathscr{S}_{b, N, \eta}^{a, M}\right)^{\prime}$ при любых $b$ и $N$. Аналогично, $f \in\left(\mathscr{S}_{b, N}\right)^{\prime}$, если при некотором $\eta>0$ функционал $f$ принадлежит $\left(\mathscr{S}_{b, N, \eta}^{a, M}\right)^{\prime}$ при любых $a$ и $M$.

Заметим, что если $f(t) \in \mathscr{L}_{1}^{\text {loc }}(0,+\infty)$ и выполнены оценки

$$
|f(t)|<c t^{a} \quad \text { при } t>t_{0}, \quad|f(t)|<c t^{b} \quad \text { при } 0<t<t_{0}
$$

с некоторыми $c, a, b$ и $t_{0}>0$, то обобщенная функция $f(t)$, определяемая формулой

$$
(f(t), \varphi(t))=\int_{0}^{\infty} f(t) \varphi(t) d t, \quad \varphi(t) \in \underset{0}{\mathscr{S}_{b, 0, \delta}^{a, 0}},
$$

принадлежит пространству $\left(\underset{\mathscr{S}_{0}^{a, 0}}{b, 0, \delta}\right)^{\prime}$ с любым $\delta>0$.

При $a>b$ преобразование Меллина основных функций $\varphi(t) \in \mathscr{S}_{b, N, \delta}^{a, M}$ определяется формулой

$$
\mathscr{M}[\varphi] \equiv \widehat{\varphi}(z)=\int_{0}^{+\infty} t^{-i z}\left[\varphi(t)-T_{\varphi}^{\langle y\rangle}(t)\right] d t, \quad z=x+i y \in \bar{\Pi}_{b}^{a}
$$

(см. [2]). Этот интеграл корректно определен при $b \leqslant y \leqslant a$, кроме, возможно, целых отрицательных значений $y$, находящихся между $a$ и $b$, и определяет в полосе $\Pi_{b}^{a}$ аналитическую функцию, имеющую, быть может, простые полюсы в точках $z=-i k, k=1,2, \ldots$, попавших в эту полосу. Вне полюсов, скажем в $\bar{\Pi}_{b}^{a} \cap$ $\{|x|>1\}$, функция $|\widehat{\varphi}(z)|$ ограничена. Легко проверяется, что для любого целого $p \leqslant \min \{N, M\}$ имеем $\left(t \frac{d}{d t}\right)^{p} \varphi \in \mathscr{S}_{b, N-p, \delta}^{a, M-p}$ и

$$
\mathscr{M}\left[\left(t \frac{d}{d t}\right)^{p} \varphi(t)\right]=(i z-1)^{p} \widehat{\varphi}(z) .
$$

Отсюда вытекает, что $|\widehat{\varphi}(z)|$ убывает в полосе $\bar{\Pi}_{b}^{a}$ при $|z| \rightarrow \infty$ по крайней мере $\operatorname{KaK}|z|^{-p}$.

ОПРЕДЕЛЕНИЕ 2. Рассмотрим функции $\widehat{\varphi}(z)$, аналитические в полосе $\Pi_{b}^{a}$, за исключением, быть может, целочисленных отрицательных точек чисто мнимой оси $z_{k}=-i k, k=1,2, \ldots, r$, где они могут иметь простые полюсы. Пусть $Q$ - некоторое вещественное число. Через $H_{Q}\left(\Pi_{b}^{a}\right)$ будем обозначать банахово пространство таких функций с нормой

$$
\|\widehat{\varphi}(z)\|_{H_{Q}}=\sup _{z \in \Pi_{b}^{a}}\left|\widehat{\varphi}(z)(1+|z|)^{Q-r} \Theta_{b}^{a}(z)\right|,
$$


где $\Theta_{b}^{a}(z)=\prod_{k=1}^{r}\left(z-z_{k}\right)$, или, что то же самое,

$$
\Theta_{b}^{a}(z)= \begin{cases}\prod_{\ell=1}^{\langle b\rangle+1}(z-i \ell), & \text { если } b<-1, a>-1, \\ \prod_{\ell=\langle a\rangle+2}^{\langle b\rangle+1}(z-i \ell), & \text { если } b<a<-1 .\end{cases}
$$

Через $H_{\infty}\left(\Pi_{b}^{a}\right)$ обозначаем проективньй предел (пересечение) всех пространств $H_{Q}\left(\Pi_{b}^{a}\right)$.

Таким образом, преобразование Меллина осуществляет непрерывное отображение

$$
\mathscr{M}: \mathscr{S}_{b, M}^{a, M} \mapsto H_{M}\left(\Pi_{b}^{a}\right) .
$$

Для $\varphi(t) \in \mathscr{S}_{+}$преобразование Меллина $\widehat{\varphi}(z)$ есть аналитическая функция в комплексной плоскости, кроме, может быть, точек $-i k, k=1,2, \ldots$, в которых у нее простые полюсы с вычетами $\frac{1}{(k-1) !} \varphi^{(k-1)}(0)$, и для любых $a>b \widehat{\varphi}(z) \in$ $H_{\infty}\left(\Pi_{b}^{a}\right)$.

Если $\varphi(t) \in \mathscr{S}_{0}^{a}$, то $\widehat{\varphi}(z)$ есть аналитическая функция в $\Pi_{b}^{a}$, убывающая быстрее любой степени $|z|^{-1}$ при $|z| \rightarrow \infty$.

Если $a<-1$, то преобразование Меллина имеет нетривиальное ядро. Это ядро состоит из всех многочленов степени $\langle a\rangle$.

Если $\widehat{\varphi}(z) \in H_{\infty}\left(\Pi_{b}^{a}\right)$, то формула

$$
\mathscr{M}^{-1}[\widehat{\varphi}(z)]=\frac{1}{2 i \pi} \int_{L_{0}} t^{i z-1} \widehat{\varphi}(z) d z,
$$

где $L_{0}=\left\{z=x+i y_{0}:|x|<\infty,[a]<y_{0}<a, b<y_{0}\right\}$, определяет функцию из $\stackrel{0}{\mathscr{S}}_{b+\varepsilon}^{a-\varepsilon}$ (для любого сколь угодно малого $\varepsilon$ ), преобразование Меллина которой совпадает с $\widehat{\varphi}(z)$.

Обратное преобразование Меллина $\mathscr{M}^{-1}$ осуществляет непрерьвное отображение

$$
\mathscr{M}^{-1}: H_{M+2}\left(\Pi_{b}^{a}\right) \mapsto \stackrel{0}{\mathscr{S}}_{b+\varepsilon, M}^{a-\varepsilon, M},
$$

где $\varepsilon>0$ - любое сколь угодно малое число. Преобразование Меллина и обратное к нему осуществляют непрерьвные отображения

$$
\mathscr{M}: \mathscr{S}_{b}^{a} \mapsto H_{\infty}\left(\Pi_{b}^{a}\right) ; \quad \mathscr{M}^{-1}: H_{\infty}\left(\Pi_{b}^{a}\right) \mapsto \stackrel{0}{\mathscr{S}}_{b+\varepsilon}^{a-\varepsilon}
$$

Если полюсов у $\widehat{\varphi}(z)$ в полосе $\Pi_{b}^{a}$ нет, то $\varphi(t) \in \underset{0}{\mathscr{S}^{a-\varepsilon}}{ }^{a-\varepsilon}$.

Мультипликативная свертка основных функций $\varphi_{1}$ и $\varphi_{2}$ из $\mathscr{S}_{+}$определяется формулой

$$
\psi(t)=\int_{0}^{\infty} \varphi_{1}\left(\frac{t}{\tau}\right) \varphi_{2}(\tau) \frac{d \tau}{\tau}=\int_{0}^{\infty} \varphi_{1}(\tau) \varphi_{2}\left(\frac{t}{\tau}\right) \frac{d \tau}{\tau} .
$$

Функция $\psi(t)$ определена и бесконечно дифференцируема при $t \in(0,+\infty)$. Если $\varphi_{1}(t) \in{ }_{q} \mathscr{S}_{+}$, а $\varphi_{2} \in{ }_{p} \mathscr{S}_{+}, p>q$, то $\psi^{(j)}(t)$ непрерывны на $[0,+\infty)$ для $j=$ $0,1, \ldots, p$, причем $\psi^{(j)}(0)=0$ при $j=0,1, \ldots, q$.

В дальнейшем мультипликативную свертку будем обозначать следующим образом: $\psi=\varphi_{1} * \varphi_{2}$. Справедливо следующее 
УТВЕРЖДЕНИЕ 1. Операчия свертки (2.16) определяет непрерывное билинейное отображение

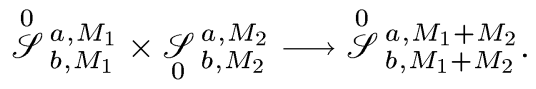

При этом справедлива формула

$$
\widehat{\psi}(z)=\widehat{\varphi}_{1}(z) \cdot \widehat{\varphi}_{2}(z), \quad b \leqslant \operatorname{Im} z \leqslant a
$$

(см. далее лемму П2).

\section{§ 3. Семейства основных функций}

В этом параграфе мы рассмотрим пространства семейств основных функций и их последовательностей. Пусть $\mathbb{B}$ - банахово пространство функций $\psi$ с нормой $\mathscr{P}[\psi]$ и $I$ - счетное множество индексов.

ОПРЕДЕЛЕНИЕ 3. Рассмотрим семейство функций $\varphi(t)=\left\{\varphi^{\beta}(t) \in \mathbb{B}, \beta \in I\right\}$. В линейном пространстве этих семейств введем две нормы:

$$
{ }_{I} \mathscr{P}[\varphi(t)]=\sum_{\beta \in I} \mathscr{P}\left[\varphi^{\beta}(t)\right], \quad{ }^{I} \mathscr{P}[\varphi(t)]=\sup _{\beta \in I} \mathscr{P}\left[\varphi^{\beta}(t)\right] .
$$

Банаховы пространства с этими нормами будем обозначать $I_{\mathbb{B}}$ и ${ }^{I} \mathbb{B}$ соответственно.

Так, например, если $\varphi(t)=\left\{\varphi^{\beta}(t) \in \mathscr{S}_{b, N, \delta}^{a, M}, \beta \in I\right\}$, то ${ }_{I} \mathscr{S}_{b, N, \delta}^{a, M}$ обозначает пространство таких семейств с нормой

$$
{ }_{I} \mathscr{P}_{b, N, \delta}^{a, M}[\varphi(t)]=\sum_{\beta \in I} \mathscr{P}_{b, N, \delta}^{a, M}\left[\varphi^{\beta}(t)\right] .
$$

Аналогично,

$$
\varphi \in{ }^{I} \mathscr{S}_{b, N, \delta}^{a, M}, \quad \text { если } \quad{ }^{I} \mathscr{P}_{b, N, \delta}^{a, M}[\varphi]=\sup _{\beta \in I} \mathscr{P}_{b, N, \delta}^{a, M}\left[\varphi^{\beta}(t)\right]<\infty .
$$

В этом случае будем также говорить, что семейство $\varphi(t)$ ограничено по $\beta \in I$.

Каждое семейство $\left\{\varphi^{\beta} \in \mathscr{S}_{b, M}^{a, M}, \beta \in I\right\}=\varphi(t) \in{ }^{I} \mathscr{S}_{b, M}^{a, M}, b<a$, для любого $d \in(b, a)$ определяет непрерьвньй оператор

$$
B_{0}:{ }_{I} \mathscr{S}_{0}^{a, M_{1}} \mapsto M_{1}^{0} \mathscr{S}_{d, M+M_{1}}^{a, M+M_{1}},
$$

действуюший по формуле

$$
B_{0} \omega=\sum_{\beta \in I} \omega^{\beta} * \stackrel{0}{\varphi}^{\beta}=\sum_{\beta \in I} \int_{0}^{\infty} \omega^{\beta}(\varkappa)^{0} \varphi^{\beta}\left(\frac{t}{\varkappa}\right) \frac{d \varkappa}{\varkappa} .
$$

Действительно, в силу утверждения 1 имеем

$$
\begin{aligned}
\mathscr{P}_{d, M+M_{1}}^{a, M+M_{1}}\left[B_{0} \omega\right] & \leqslant \sum_{\beta \in I} \mathscr{P}_{d, M+M_{1}}^{a, M+M_{1}}\left[\omega^{\beta} * \stackrel{\varphi}{\varphi}^{\beta}\right] \leqslant \sum_{\beta \in I} \mathscr{P}_{d, M_{1}}^{a, M_{1}}\left[\omega^{\beta}\right] \mathscr{P}_{b, M}^{a, M}\left[\stackrel{0}{\varphi}^{\beta}\right] \\
& \leqslant \sup _{\beta \in I} \mathscr{P}_{b, M}^{a, M}\left[\stackrel{\varphi}{\varphi}^{\beta}\right] \sum_{\beta \in I} \mathscr{P}_{d, M_{1}}^{a, M_{1}}\left[\omega^{\beta}\right] \\
& \leqslant{ }^{I} \mathscr{P}_{b, M}^{a, M}[\stackrel{0}{\varphi}] \cdot{ }_{I} \mathscr{P}_{d, M_{1}}^{a, M_{1}}[\omega] \leqslant \text { const }_{I} \mathscr{P}_{d, M_{1}}^{a, M_{1}}[\omega] .
\end{aligned}
$$


ОПРЕДЕЛЕНИЕ 4 . Если $\frac{d^{j}}{d t^{j}} \varphi^{\beta}(0)=0$ при всех $\beta \in I$, то мы говорим, что $j$-я производная семейства $\left\{\varphi^{\beta}(t) \in \mathscr{S}_{b, N, \delta}^{a, M}, \quad \beta \in I\right\}=\varphi(t)$ в нуле равна нулю, и пишем $\varphi^{(j)}(0)=0$.

Пусть задано семейство $\left\{\widehat{\varphi}^{\beta}(z) \in H_{M}\left(\Pi_{b}^{a}\right), \beta \in I\right\}=\widehat{\varphi}(z)$. Точка $z_{0} \in \Pi_{b}^{a}$ называется нулем семейства $\widehat{\varphi}(z)$ кратности $r$, если

$$
\begin{gathered}
\forall \beta \in I \quad \widehat{\varphi}^{\beta}\left(z_{0}\right)=0, \quad \frac{d}{d z} \widehat{\varphi}^{\beta}\left(z_{0}\right)=0, \quad \ldots, \quad \frac{d^{r-1}}{d z^{r-1}} \widehat{\varphi}^{\beta}\left(z_{0}\right)=0, \\
\frac{d^{r}}{d z^{r}} \widehat{\varphi}^{\beta}\left(z_{0}\right) \neq 0 \quad \text { для некоторого } \beta \in I .
\end{gathered}
$$

Мы будем рассматривать направленные множества семейств основных функций $\varphi_{\lambda}(t)=\left\{\varphi_{\lambda}^{\beta}(t) \in \mathscr{S}_{b, N, \delta}^{a, M}, \quad \beta \in I\right\}, \lambda \in \mathbb{R}_{+}$, и будем называть такие множества семействами последовательностей основных функций.

ОПРеДЕлЕниЕ 5. Пусть $\left\{\varphi_{\lambda}^{\beta} \in \mathbb{B}, \beta \in I\right\}, \lambda>0,-$ семейство последовательностей элементов некоторого банахова пространства $\mathbb{B}$. Будем говорить, что семейство последовательностей $\left\{\varphi_{\lambda}^{\beta}\right\}$ ограниченно по $\beta \in I$ сходится к семейству $\left\{\varphi_{0}^{\beta}\right\}$ при $\lambda \rightarrow+\infty$, если:

1) $\varphi_{\lambda}^{\beta} \longrightarrow \varphi_{0}^{\beta}, \lambda \rightarrow+\infty, \forall \beta \in I$;

2) сушествуют постоянные $c$ и $\lambda_{0}$, не зависяшие от $\beta$, такие, что

$$
\left\|\varphi_{\lambda}^{\beta}\right\|_{\mathbb{B}}<c, \quad \lambda>\lambda_{0}
$$

ПримеР 1. Пусть $\beta$ пробегает счетное множество $I$ точек интервала $(0, \pi)$, причем концы интервала есть предельные точки $I$. Рассмотрим множество функций $\varphi^{\beta}(t)=e^{i(\cos \beta+i \sin \beta) t}$. Очевидно, что $\varphi^{\beta}(t) \in \mathscr{S}_{b, N, \delta}^{a, M}$ при любых $M$ и $N$. Нетрудно проверить, что для достаточно большого $Q$ (например, $Q \geqslant a+M+1$ )

$$
\sup _{\beta \in I} \mathscr{P}_{b, N, \delta}^{a, M}\left[(\sin \beta)^{Q} e^{i(\cos \beta+i \sin \beta) t}\right] \leqslant \text { const },
$$

а потому семейство функций

$$
\left\{(\sin \beta)^{Q} e^{i t e^{i \beta}} \in \mathscr{S}_{b}^{a}, \quad \beta \in I\right\} \equiv \varphi(t) \in{ }^{I} \mathscr{S}_{b, N, \delta}^{a, M}
$$

при любых $M, N$ и $\delta$.

ОПРЕДЕЛЕНИЕ 6 . Пусть $q$ - целое неотрицательное число. Рассмотрим семейства последовательностей $\varphi_{\lambda}(t)=\left\{\varphi_{\lambda}^{\beta}(t) \in \mathscr{S}_{b, N, \delta}^{a, M}, \lambda>0, \beta \in I\right\}$ со свойствами:

1) $\varphi_{\lambda}^{\beta}(t)$ - измеримые функции по совокупности переменных $t, \lambda$, которые $q$ раз непрерывно дифференцируемы по $\lambda$ (в смысле $\Phi$ реше) как отображение $\mathbb{R}_{+} \rightarrow$ $\mathscr{S}_{b, N, \delta}^{a, M}, \beta \in I$

2) имеет место

$$
I \mathscr{P}_{(b, N, \delta)}^{q(a, M)}\left[\varphi_{\lambda}^{\beta}(t)\right] \equiv \sup _{\beta \in I} \max _{j \leqslant q} \sup _{\lambda \in \mathbb{R}_{+}} \mathscr{P}_{b, N, \delta}^{a, M}\left[\left(\lambda \frac{d}{d \lambda}\right)^{j} \varphi_{\lambda}^{\beta}(t)\right]<\infty .
$$

Линейное пространство семейств последовательностей, введенных в определении 6, с нормой ${ }^{I} \mathscr{P}_{(b, N, \delta)}^{q(a, M)}\left[\varphi_{\lambda}^{\beta}(t)\right]$ будем обозначать ${ }^{I} F^{q}\left[\mathscr{S}_{b, N, \delta}^{a, M}\right]$. 
ЗАмЕЧАНИЕ. Если множество $I$ состоит только из одного элемента, то символ $I$ в обозначениях соответствующих пространств будет опушен. Например, через $F^{q}\left[\mathscr{S}_{b, N, \delta}^{a, M}\right]$ обозначаем банахово пространство последовательностей функций $\varphi_{\lambda}(t)$ с нормой

$$
\mathscr{P}_{(b, N, \delta)}^{q(a, M)}\left[\varphi_{\lambda}(t)\right]=\max _{j \leqslant q} \sup _{\lambda \in \mathbb{R}_{+}} \mathscr{P}_{b, N, \delta}^{a, M}\left[\left(\lambda \frac{d}{d \lambda}\right)^{j} \varphi_{\lambda}(t)\right]
$$

Для $\omega(t) \in \underset{I}{\mathscr{S}_{0}} \underset{b, M}{a, M}$ и семейства последовательностей $\varphi_{\lambda}(t) \in{ }^{I} F^{M}\left[\begin{array}{c}0 \\ \mathscr{S}^{a, N} \\ b, N\end{array}\right]$ определим функцию

$$
\Psi\left[\omega, \varphi_{\lambda}\right](t)=\sum_{\beta \in I} \psi\left[\omega^{\beta}, \varphi_{\lambda}^{\beta}\right](t)
$$

где каждая функция

$$
\psi\left[\omega^{\beta}, \varphi_{\lambda}^{\beta}\right](t)=\int_{0}^{\infty} \omega^{\beta}(\varkappa) \varphi_{\varkappa}^{\beta}\left(\frac{t}{\varkappa}\right) \frac{d \varkappa}{\varkappa}, \quad \beta \in I,
$$

согласно лемме П2 корректно определена и принадлежит $\stackrel{0}{\mathscr{S}} \underset{b, M+N}{a, M+N}$. Следующая важная лемма обобщает утверждение 1.

ЛЕмма 2. Билинейное отображение

$$
\underset{0}{\mathscr{S}_{b, M}^{a, M}} \times{ }^{I} F^{M}\left[\begin{array}{c}
0 \\
\mathscr{S}_{b, N}^{a, N} \\
b
\end{array}\right] \stackrel{\Psi}{\longrightarrow} \stackrel{\mathscr{S}}{b, M+N}_{b, M+N}^{a, M}
$$

корректно определено и непрерывно.

ДокАЗАТЕЛьСТвО. Пользуясь леммой П2, имеем

$$
\begin{aligned}
\mathscr{P}_{b, M+N}^{a, M+N}\left[\Psi\left[\omega, \varphi_{\lambda}\right](t)\right] & =\sum_{\beta \in I} \mathscr{P}_{b, M+N}^{a, M+N}\left[\int_{0}^{\infty} \omega^{\beta}(\varkappa) \varphi_{\varkappa}^{\beta}\left(\frac{t}{\varkappa}\right) \frac{d \varkappa}{\varkappa}\right] \\
& \leqslant \sum_{\beta \in I} \operatorname{const} \mathscr{P}_{b, M}^{a, M}\left[\omega^{\beta}\right] \mathscr{P}_{(b, N)}^{M(a, N)}\left[\varphi_{\lambda}^{\beta}\right] \\
& \leqslant \text { const } \sup _{\beta \in I} \mathscr{P}_{(b, N)}^{M(a, N)}\left[\varphi_{\lambda}^{\beta}\right] \sum_{\beta \in I} \mathscr{P}_{b, M}^{a, M}\left[\omega^{\beta}\right] \\
& =\text { const }^{I} \mathscr{P}_{(b, N)}^{M(a, N)}\left[\varphi_{\lambda}^{\beta}\right] \cdot{ }_{I} \mathscr{P}_{b, M}^{a, M}[\omega] .
\end{aligned}
$$

Согласно той же лемме

$$
\left(\psi\left[\omega^{\beta}, \varphi_{\lambda}^{\beta}\right]\right)^{(j)}(0)=0 \text { для } j=0,1, \ldots,\langle a\rangle
$$

и для всех $\beta \in I$. Поэтому $\Psi\left[\omega, \varphi_{\lambda}\right](t) \in \stackrel{0}{\mathscr{S}}_{b, M+N}^{a, M+N}$. Лемма доказана. 
Пусть семейство последовательностей

$$
\varphi_{\lambda}(t)=\left\{\varphi_{\lambda}^{\beta}(t) \in \mathscr{S}_{b, N}^{a, N}, \quad \beta \in I, \quad \lambda>0\right\} \in{ }^{I} F^{M}\left[\mathscr{S}_{b, N}^{a, N}\right] .
$$

Определим семейство линейных операторов, связанное с семейством последовательностей $\varphi_{\lambda}(t)$,

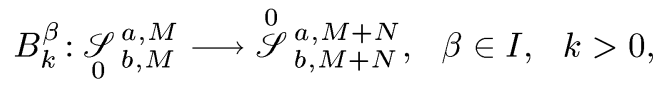

формулой

$$
B_{k}^{\beta} \omega \equiv \psi\left[\omega, \varphi_{k \lambda}^{\beta}\right](t)=\int_{0}^{\infty} \omega(\varkappa) \stackrel{\varphi}{\varphi}_{k \varkappa}^{\beta}\left(\frac{t}{\varkappa}\right) \frac{d \varkappa}{\varkappa}, \quad \beta \in I
$$

где ${ }_{\varphi}^{0}{ }_{\lambda}^{\beta}(t)=\varphi_{\lambda}^{\beta}(t)-T_{\varphi_{\lambda}^{\beta}(t)}^{\langle a\rangle}(t)$.

СлеДСТвИЕ 1. Для любого $k>0 u \beta \in I$ оператор (3.13) корректно определен, причем

$$
\mathscr{P}_{b, M+N}^{a, M+N}\left[B_{k}^{\beta} \omega\right] \leqslant \mathrm{const} \cdot{ }^{I} \mathscr{P}_{(b, N)}^{M(a, N)}\left[\varphi_{\varphi}^{0}(t)\right] \cdot \mathscr{P}_{b, M}^{a, M}[\omega], \quad \omega \in \mathscr{\mathscr { S }}_{0}^{a, M}, a,
$$

əде const не зависит от $\beta, k u \varphi_{\lambda}(t)$.

Действительно, по лемме П2 имеем

$$
\mathscr{P}_{b, M+N}^{a, M+N}\left[B_{k}^{\beta} \omega\right] \leqslant \mathrm{const} \mathscr{P}_{(b, N)}^{M(a, N)}\left[\stackrel{0}{\varphi}_{k \lambda}^{\beta}(t)\right] \cdot \mathscr{P}_{b, M}^{a, M}[\omega], \quad \beta \in I .
$$

Далее, в силу (3.12)

$$
\begin{aligned}
\mathscr{P}_{(b, N)}^{M(a, N)}\left[\stackrel{0}{\varphi}_{k \lambda}^{\beta}(t)\right] & =\max _{j \leqslant M} \sup _{\lambda \in \mathbb{R}_{+}} \mathscr{P}_{b, N}^{a, N}\left[\left(\lambda \frac{d}{d \lambda}\right)^{j} \varphi^{0} \varphi_{k \lambda}^{\beta}(t)\right] \\
& \leqslant \max _{j \leqslant M} \sup _{k \lambda \in \mathbb{R}_{+}} \mathscr{P}_{b, N}^{a, N}\left[\left(k \lambda \frac{d}{d k \lambda}\right)^{j} \varphi^{0} \varphi_{k \lambda}^{\beta}(t)\right] \\
& \leqslant \sup _{\beta \in I} \max _{j \leqslant M} \sup _{\lambda \in \mathbb{R}_{+}} \mathscr{P}_{b, N}^{a, N}\left[\left(\lambda \frac{d}{d \lambda}\right)^{j} \varphi^{0} \varphi_{\lambda}^{\beta}(t)\right]={ }^{I} \mathscr{P}_{(b, N)}^{M(a, N)}\left[\stackrel{0}{\varphi}_{\lambda}(t)\right] .
\end{aligned}
$$

Следствие доказано.

Определим оператор $B_{k}:{ }_{I} \mathscr{S}_{0}^{a, M}, M \stackrel{0}{\mathscr{S}_{b, M+N}^{a, M+N}}$ формулой

$$
B_{k} \omega \equiv \sum_{\beta \in I} B_{k}^{\beta} \omega^{\beta}
$$

Непосредственно из (3.15) получаем

СледСТвиЕ 2. Оператор $B_{k}$, построенный по семейству последовательностей (3.12) и определяемый формулой (3.16), непрерывен, причем

$$
\mathscr{P}_{b, M+N}^{a, M+N}\left[B_{k} \omega\right] \leqslant \mathrm{const} \cdot{ }^{I} \mathscr{P}_{(b, N)}^{M(a, N)}\left[\varphi_{\varphi_{\lambda}}(t)\right] \cdot{ }_{I} \mathscr{P}_{b, M}^{a, M}[\omega],
$$

әде const не зависит от $k u \varphi_{\lambda}(t)$. 


\section{§ 4. Квазиасимптотика обобщенных функций}

Пусть $f \in\left(\mathscr{S}_{b, N, \eta}^{a, M}\right)^{\prime}$ и $\varphi \in \mathscr{S}_{b, N, \delta}^{a, M}$. В этом параграфе и всюду далее будем считать $M \leqslant N$. Тогда при больших $k$ имеет смысл выражение $(f(k t), \varphi(t))=$ $(1 / k)(f(t), \varphi(t / k))$.

Действительно, при $k \delta>\eta$ функция $\varphi(t / k) \in \mathscr{S}_{b, N, k \delta}^{a, M} \subset \mathscr{S}_{b, N, \eta}^{a, M}$.

Пусть $\varphi_{k}(t)=\left\{\varphi_{k}^{\beta}(t) \in \mathscr{S}_{b, N, \delta}^{a, M}, \quad \beta \in I, k>0\right\}$. Мы говорим, что $f(t)$ обладает квазиасимптотикой на семействе последовательностей $\varphi_{k}(t)$ относительно автомодельной функции $\rho(k)$, если

$$
\frac{1}{\rho(k)}\left(f(k t), \varphi_{k}^{\beta}(t)\right) \longrightarrow c^{\beta}, \quad k \rightarrow \infty, \quad \forall \beta \in I .
$$

Напомним, что наша задача - найти условия, при которых из (1.4) или (4.1) следовало бы существование квазиасимптотики у $f(t)$ относительно автомодельной функции $\rho(k)$, т.е. $(1.5)$.

В этом параграфе мы приведем некоторые предварительные результаты.

Лемма 3. Пусть $f \in\left(\mathscr{S}_{b, N, \eta}^{a, M}\right)^{\prime} u \varphi \in \mathscr{S}_{b, N, \delta}^{a, M}$, где $M \leqslant N, b<a$. Тогда существуют постоянные $C$ и $k_{0}$ такие, что

$$
\left|(f(k t), \varphi(t))-\sum_{j=0}^{\langle a\rangle} \frac{1}{k^{j+1}} \frac{\varphi^{(j)}(0)}{j !}\left(f(t), t^{j}\right)\right| \leqslant C k^{a} \mathscr{P}_{b, N, \delta}^{a, M}[\varphi] \quad n p u \quad k>k_{0}
$$

Если а > -1, то сумма в (4.2) отсутствует.

ДокАЗАТЕЛЬСТво. Учитывая, что

$$
\begin{aligned}
T_{\varphi(t / k)}^{\langle a\rangle}(t) & =T_{\varphi(t)}^{\langle a\rangle}\left(\frac{t}{k}\right), \\
T_{T_{\varphi(t / k)}^{\langle a\rangle}(t)}^{\langle b\rangle}(t) & =T_{T_{\varphi(t)}^{\langle a\rangle}(t / k)}^{\langle b\rangle}(t)=T_{T_{\varphi}^{\langle a\rangle}}^{\langle b\rangle}\left(\frac{t}{k}\right)=T_{\varphi}^{\langle a\rangle}\left(\frac{t}{k}\right),
\end{aligned}
$$

и обозначая $\psi(t)=\varphi(t)-T_{\varphi(t)}^{\langle a\rangle}(t)$, имеем

$$
\begin{aligned}
\left|(f(k t), \varphi(t))-\sum_{j=0}^{\langle a\rangle} \frac{1}{k^{j+1}} \frac{\varphi^{(j)}(0)}{j !}\left(f(t), t^{j}\right)\right| \\
=\left|\frac{1}{k}\left(f(t), \varphi\left(\frac{t}{k}\right)\right)-\frac{1}{k}\left(f(t), T_{\varphi(t / k)}^{\langle a\rangle}(t)\right)\right| \\
=\frac{1}{k}\left|\left(f(t), \psi\left(\frac{t}{k}\right)\right)\right| \leqslant \frac{\text { const }}{k} \mathscr{P}_{b, N, \eta}^{a, M}\left[\psi\left(\frac{t}{k}\right)\right] \\
\leqslant \frac{\text { const }}{k}\left[\max _{j \leqslant N} \int_{0}^{\eta} t^{b} \mid t^{j} \frac{d^{j}}{d t^{j}}\left\{\varphi\left(\frac{t}{k}\right)-T_{\varphi(t / k)}^{\langle a\rangle}(t)\right.\right. \\
\left.\quad-\left(T_{\varphi(t / k)}^{\langle b\rangle}(t)-T_{T_{\varphi(t / k)}^{\langle b\rangle}(t)}^{\langle b\rangle}(t)\right)\right\} \mid d t
\end{aligned}
$$




$$
\begin{aligned}
&+ \max _{j \leqslant M} \int_{\eta}^{\infty} t^{a}\left|t^{j} \frac{d^{j}}{d t^{j}}\left\{\varphi\left(\frac{t}{k}\right)-T_{\varphi(t / k)}^{\langle a\rangle}(t)\right\}\right| d t \\
&+\left.\sum_{j=0}^{\langle b\rangle}\left|\left(\varphi\left(\frac{t}{k}\right)-T_{\varphi(t / k)}^{\langle a\rangle}(t)\right)^{(j)}\right|_{t=0} \mid\right] \\
&=\frac{\mathrm{const}}{k}\left[\max _{j \leqslant N} \int_{0}^{\eta} t^{b}\left|t^{j} \frac{d^{j}}{d t^{j}}\left\{\varphi\left(\frac{t}{k}\right)-T_{\varphi}^{\langle b\rangle}\left(\frac{t}{k}\right)\right\}\right| d t\right. \\
&+\max _{j \leqslant M} \int_{\eta}^{\infty} t^{a}\left|t^{j} \frac{d^{j}}{d t^{j}}\left\{\varphi\left(\frac{t}{k}\right)-T_{\varphi}^{\langle a\rangle}\left(\frac{t}{k}\right)\right\}\right| d t \\
&\left.+\sum_{j=\langle a\rangle+1}^{\langle b\rangle}\left|\left(\varphi\left(\frac{t}{k}\right)\right)^{(j)}\right|\right] .
\end{aligned}
$$

Делая замену переменных $t / k=t^{\prime}$ (опуская штрихи), при $k>\eta / \delta$ имеем

$$
\begin{aligned}
& \frac{1}{k}\left|\left(f(t), \psi\left(\frac{t}{k}\right)\right)\right| \leqslant \text { const } \cdot k^{a}\left[\max _{j \leqslant N} k^{b-a} \int_{0}^{\eta / k} t^{b}\left|t^{j} \frac{d^{j}}{d t^{j}}\left\{\varphi(t)-T_{\varphi}^{\langle b\rangle}(t)\right\}\right| d t\right. \\
& \left.+\max _{j \leqslant M} \int_{\eta / k}^{\infty} t^{a}\left|t^{j} \frac{d^{j}}{d t^{j}}\left\{\varphi(t)-T_{\varphi}^{\langle a\rangle}(t)\right\}\right| d t+k^{-a} \sum_{j=\langle a\rangle+1}^{\langle b\rangle} \frac{1}{k^{j+1}}\left|\varphi^{(j)}(0)\right|\right] \\
& \leqslant \text { const } \cdot k^{a}\left[\max _{j \leqslant N} \int_{0}^{\delta} t^{b}\left|t^{j} \frac{d^{j}}{d t^{j}}\left\{\varphi(t)-T_{\varphi}^{\langle b\rangle}(t)\right\}\right| d t\right. \\
& \left.+\max _{j \leqslant M} \int_{0}^{\infty} t^{a}\left|t^{j} \frac{d^{j}}{d t^{j}}\left\{\varphi(t)-T_{\varphi}^{\langle a\rangle}(t)\right\}\right| d t+\sum_{j=\langle a\rangle+1}^{\langle b\rangle} \frac{1}{k^{a+1+j}}\left|\varphi^{(j)}(0)\right|\right] .
\end{aligned}
$$

Первое слагаемое в правой части последнего из неравенств (4.3) непосредственно оценивается нормой $\mathscr{P}_{b, N, \delta}^{a, M}[\varphi]$, третье слагаемое, в силу неравенства $a+j+1 \geqslant a+$ $\langle a\rangle+2>0$ (см. (1.3)) оценивается той же нормой. Оценим второе слагаемое. Разобьем интеграл на два интеграла: от 0 до $\delta$ и от $\delta$ до $\infty$. Имеем

$$
\begin{aligned}
& \max _{j \leqslant M} \int_{0}^{\delta} t^{a}\left|t^{j} \frac{d^{j}}{d t^{j}}\left\{\varphi(t)-T_{\varphi}^{\langle a\rangle}(t)\right\}\right| d t \\
& \leqslant \max _{j \leqslant M} \int_{0}^{\delta} t^{a}\left|t^{j} \frac{d^{j}}{d t^{j}}\left\{\varphi(t)-T_{\varphi}^{\langle b\rangle}(t)\right\}\right| d t \\
& \quad+\max _{j \leqslant M} \int_{0}^{\delta} t^{a}\left|t^{j} \frac{d^{j}}{d t^{j}}\left\{T_{\varphi}^{\langle b\rangle}(t)-T_{\varphi}^{\langle a\rangle}(t)\right\}\right| d t \\
& \leqslant c \cdot \max _{j \leqslant N} \int_{0}^{\delta} t^{b}\left|t^{j} \frac{d^{j}}{d t^{j}}\left\{\varphi(t)-T_{\varphi}^{\langle b\rangle}(t)\right\}\right| d t \\
& \quad+\operatorname{const} \int_{0}^{\delta} t^{a+\langle a\rangle+1} d t \sum_{j=\langle a\rangle+1}^{\langle b\rangle}\left|\varphi^{(j)}(0)\right| \leqslant \operatorname{const} \mathscr{P}_{b, N, \delta}^{a, M}[\varphi] .
\end{aligned}
$$


Здесь учтено, что $a>b, M \leqslant N$ и $a+\langle a\rangle+1>-1$ (см. (1.3)). Далее,

$$
\begin{aligned}
& \max _{j \leqslant M} \int_{\delta}^{\infty} t^{a}\left|t^{j} \frac{d^{j}}{d t^{j}}\left\{\varphi(t)-T_{\varphi}^{\langle a\rangle}(t)\right\}\right| d t \\
& \quad \leqslant \max _{j \leqslant M} \int_{\delta}^{\infty} t^{a}\left|t^{j} \frac{d^{j}}{d t^{j}} \varphi(t)\right| d t+\max _{j \leqslant M} \int_{\delta}^{\infty} t^{a+j}\left|\frac{d^{j}}{d t^{j}} T_{\varphi}^{\langle a\rangle}(t)\right| d t \\
& \quad \leqslant \mathscr{P}_{b, N, \delta}^{a, M}[\varphi]+\operatorname{const} \int_{\delta}^{\infty} t^{a+\langle a\rangle} d t \sum_{j=0}^{\langle a\rangle}\left|\varphi^{(j)}(0)\right| \leqslant \operatorname{const} \mathscr{P}_{b, N, \delta}^{a, M}[\varphi] .
\end{aligned}
$$

Здесь мы учли, что $a+\langle a\rangle<-1$ (см. (1.3)). Отсюда с учетом (4.4), объединяя все оценки, получаем (4.2). Лемма доказана.

СледСтвиЕ. Пусть $f \in\left(\mathscr{S}_{b, N, \eta}^{a, M}\right)^{\prime} ;$ тогда

$$
f(t)=\sum_{j=0}^{\langle a\rangle} c_{j} \delta^{(j)}(t)+g(t),
$$

где семейство функционалов

$$
\frac{1}{k^{a}} g(k t), \quad k \geqslant k_{0}>0, \quad \text { ограничено в } \quad\left(\mathscr{S}_{b, N, \eta}^{a, M}\right)^{\prime} .
$$

При этом

$$
c_{j}=\frac{1}{j !}\left(f(t), t^{j}\right), \quad j=0, \ldots,\langle a\rangle .
$$

Это следствие показывает, что наибольший интерес при изучении квазиасимптотики представляет случай $\alpha \leqslant a$.

Каждая обобщенная функция $f \in \mathscr{S}_{+}^{\prime}$ принадлежит пространству $\left(\mathscr{S}_{b}^{a}\right)^{\prime}$ при некоторых $a$ и $b$. Оказывается, что пространства $\left(\mathscr{S}_{b}^{a}\right)^{\prime}$ можно описать в терминах квазиасимптотического поведения обобшенных функций в нуле и на бесконечности.

УТВЕРЖДЕНИЕ 2. ДЛя того чтобъ $f(t) \in\left(\mathscr{S}_{b}^{a}\right)^{\prime}(b<a)$, необходимо $u$ достаточно, чтобь:

1) обобщенная функиия $f(t)$ квазиасимптотически ограничена относительно $k^{b}$ nри $k \rightarrow+0$;

2) существуют постоянные $c_{j}, j=0,1, \ldots,\langle a\rangle$, такие, что

$$
f(t)=\sum_{j=0}^{\langle a\rangle} c_{j} \delta^{(j)}(t)+g(t),
$$

где $g(t)$ квазиасимптотически ограничена относительно $k^{a} n p и k \rightarrow+\infty$ (если $а>-1$, то сумма $\delta$-функиий в (4.8) отсутствует).

(Подробнее см. [1].) 
ОПРЕДЕЛЕНИЕ 7. Пусть $\varphi_{k}(t)=\left\{\varphi_{k}^{\beta} \in \mathscr{S}_{b, N, \delta}^{a, M}, \beta \in I\right\}, k>k_{0} \geqslant 0$, и $\rho(k)-$ автомодельная функция. Будем говорить, что $f \in\left(\mathscr{S}_{b, N, \delta}^{a, M}\right)^{\prime}$ обладает ограниченной по $\beta \in I$ квазиасимптотикой относительно $\rho(k)$ на семействе последовательностей $\varphi_{k}(t)$, если $\frac{1}{\rho(k)}\left(f(k t), \varphi_{k}^{\beta}(t)\right)$ ограниченно по $\beta \in I$ сходится, т.е.

1) имеет место

$$
\frac{1}{\rho(k)}\left(f(k t), \varphi_{k}^{\beta}(t)\right) \underset{k \rightarrow+\infty}{\longrightarrow} c^{\beta} \quad \forall \beta \in I ;
$$

2) существует постоянная $c$, не зависящая от $\beta$, такая, что

$$
\frac{1}{\rho(k)}\left|\left(f(k t), \varphi_{k}^{\beta}(t)\right)\right|<c \text { при } k>k_{0} .
$$

Лемма 4. Пусть задань $f(t) \in\left(\mathscr{S}_{b, N, \eta}^{a, M}\right)^{\prime}, \quad b<a, \quad M \leqslant N$ и семейство последовательностей функиий

$$
\varphi_{\lambda}(t) \in{ }^{I} F^{0}\left[\mathscr{S}_{b, N, \delta}^{a, M}\right], \quad \varphi_{\lambda}(t)=\left\{\varphi_{\lambda}^{\beta}(t) \in \mathscr{S}_{b, N, \delta}^{a, M}, \beta \in I, \lambda>0\right\},
$$

причем

$$
\varphi_{\lambda}^{\beta}(t) \underset{\lambda \rightarrow+\infty}{\longrightarrow} \varphi_{0}^{\beta}(t) \quad \text { в } \quad \mathscr{S}_{b, N, \delta}^{a, M},
$$

и для любого $j=0,1, \ldots,\langle a\rangle$ существуют $\beta_{j} \in I$ такие, что

$$
\left(\varphi_{0}^{\beta_{j}}\right)^{(j)}(0) \neq 0 \text {. }
$$

Пусть $f(t)$ обладает ограниченной по $\beta \in I$ квазиасимптотикой относительно автомодельной функции $\rho(k)$ порядка $\alpha, b<\alpha<a$, на семействе последовательностей $\varphi_{k}(t)$, т.е. выполнены (4.9), (4.10).

Тогда для любого натурального $Q\rangle\langle b\rangle$ существуют обобщенная функция $f_{1}(t) \in\left(\mathscr{S}^{a, M}\right)^{\prime}$, обращающаяся в нуль в некоторой окрестности нуля, $и$ обобщенная функиия $f_{2}(t) \in\left(\mathscr{S}_{b, N}\right)^{\prime}$ с компактным носителем такие, что $f(t)=f_{1}(t)+f_{2}(t) u$

$$
\begin{aligned}
& \left(f_{1}(t), t^{j}\right)=0, \quad j=0,1, \ldots,\langle a\rangle . \\
& \left(f_{2}(t), t^{\ell}\right)=0, \quad \ell=0,1, \ldots, Q .
\end{aligned}
$$

При этом для любой непрерывной функции $\eta(k)$, равной нулю в некоторой окрестности нуля и единице при достаточно больиих $k, u$ для любой $\omega(t) \in$ $\mathscr{S}_{0}^{a, 0} \alpha, \varepsilon, 0$ любое, такое, что $\left.\alpha+\varepsilon<a\right)$ имеет место ограниченная по $\beta \in I$ сходимость:

$$
\frac{1}{\rho(k)}\left(f_{1}(k t),\left(B_{k}^{\beta} \omega\right)(t)\right) \underset{k \rightarrow+\infty}{\longrightarrow} c_{\omega}^{\beta} .
$$

Здесь семейство операторов $B_{k}^{\beta}: \mathscr{S}_{0}^{a, 0} \underset{\alpha-\varepsilon, 0}{0} \mapsto \mathscr{S}_{\alpha-\varepsilon, M}^{a, M}$ построено по семейству последовательностей $\eta(\lambda) \varphi_{\lambda}(t)$ (см. (3.13), (3.14)). При этом существуют постоянные $k_{0}$ и $A$, не зависящие от $\beta \in I$, такие, что

$$
\frac{1}{\rho(k)}\left|\left(f_{1}(k t),\left(B_{k}^{\beta} \omega\right)(t)\right)\right| \leqslant A \cdot \mathscr{P}_{\alpha-\varepsilon, 0}^{\alpha+\varepsilon, 0}[\omega], \quad k>k_{0} .
$$

(Отметим, что если $a>-1$, то условия (4.13) и (4.14) отсутствуют.) 
ЗАмЕчАниЕ. Если $\varphi_{k}^{\beta}(t) \equiv \varphi_{0}^{\beta}(t), \beta \in I$, то условие (4.13) можно опустить, все утверждения леммы останутся справедливыми, кроме условия (4.14), которое будет выполнено только для тех $j=0,1, \ldots,\langle a\rangle$, для которых найдутся $\beta_{j}$ такие, что выполняется (4.13).

ДокАЗАТЕЛЬСТво ЛЕмМЫ. Положим $g(t)=f(t) \chi(t)$, где $\chi(t) \in C^{\infty}(0,+\infty)$ такая, что $\chi(t)=0$ при $t<\eta$ и $\chi(t)=1$ при $t>\eta+1$. Пусть, далее, $\gamma(t)-$ бесконечно дифференцируемая финитная функция с носителем в $(0,+\infty)$ такая, чтобы для обобщенной функции $f_{2}(t)=f(t)-g(t)+\gamma(t)$ вьполнялись условия (4.15). Полагаем $f_{1}(t)=f(t)-f_{2}(t)$. Очевидно, что $f_{1} \in\left(\mathscr{S}^{a, M}\right)^{\prime}$, а $f_{2} \in\left(\mathscr{S}_{b, N}\right)^{\prime}$.

Докажем, что обобщенная функция $f_{1}$ удовлетворяет соотношениям (4.14). (Это нужно доказать только для случая $a<-1$.) Для этого заметим, что имеет место ограниченная по $\beta$ сходимость

$$
\frac{1}{\rho(k)}\left(f_{1}(k t), \varphi_{k}^{\beta}(t)\right) \underset{k \rightarrow+\infty}{\longrightarrow} c^{\beta}, \quad \beta \in I .
$$

Действительно, имеем

$$
\frac{1}{\rho(k)}\left(f_{1}(k t), \varphi_{k}^{\beta}(t)\right)=\frac{1}{\rho(k)}\left(f(k t), \varphi_{k}^{\beta}(t)\right)-\frac{1}{\rho(k)}\left(f_{2}(k t), \varphi_{k}^{\beta}(t)\right) .
$$

Первое слагаемое справа по условию ограниченно по $\beta \in I$ сходится. Второе слагаемое ограниченно стремится к нулю, ибо при некотором $\delta_{1}$ и любом $\varepsilon>0$ обобщенная функция $f_{2}$ принадлежит $\left(\mathscr{S}_{b, N, \delta_{1}}^{b+\varepsilon, M}\right)^{\prime}$ и согласно лемме 3 , с учетом (4.15), выполнено соотношение

$$
\frac{1}{\rho(k)}\left|\left(f_{2}(k t), \varphi_{k}^{\beta}(t)\right)\right| \leqslant c \frac{k^{b+\varepsilon}}{\rho(k)} \mathscr{P}_{b, N, \delta}^{b+\varepsilon, M}\left[\varphi_{k}^{\beta}\right]<\mathrm{const} \frac{k^{b+\varepsilon}}{\rho(k)} \underset{k \rightarrow+\infty}{\longrightarrow} 0 .
$$

Далее, учитьвая, что $f_{1} \in\left(\mathscr{S}_{b, N, \delta_{2}}^{a, M}\right)^{\prime}$ при некотором $\delta_{2}>0$, по той же лемме 3 имеем

$$
\frac{1}{\rho(k)}\left(f_{1}(k t), \varphi_{k}^{\beta}(t)\right)=\sum_{j=0}^{\langle a\rangle} \frac{1}{\rho(k) k^{j+1}} \frac{\left(\varphi_{k}^{\beta}\right)^{(j)}(0)}{j !}\left(f_{1}(t), t^{j}\right)+O(1) \frac{k^{a}}{\rho(k)} .
$$

Отсюда и из $(4.12),(4.13)$ и (4.18) с учетом того, что $\alpha<a$, следует $(4.14)^{2}$.

Осталось доказать (4.16) и (4.17). Заметим сначала, что имеет место ограниченная по $\beta \in I$ сходимость

$$
\frac{1}{\rho(k)}\left(f_{1}(k t), \stackrel{0}{\varphi}_{k}^{\beta}(t)\right) \underset{k \rightarrow+\infty}{\longrightarrow} c^{\beta}, \quad \beta \in I,
$$

где $\stackrel{0}{\varphi}_{k}^{\beta}(t)=\varphi_{k}^{\beta}(t)-T_{\varphi_{\lambda}^{\beta}(t)}^{\langle a\rangle}(t)$. Действительно, в силу уже доказанного соотношения (4.14) левая часть (4.19) совпадает с левой частью (4.18).

\footnotetext{
${ }^{2}$ Если $\varphi_{k}^{\beta}(t) \equiv \varphi_{0}^{\beta}(t), \beta \in I$, то отсюда же следует справедливость замечания.
} 
Пусть $\eta(k)$ - произвольная непрерывная функция такая, что $\eta(k)=0$ при $k<k_{1}$ и $\eta(k)=1$ при $k>k_{2}>k_{1}$. Введем семейство функций $\Phi_{\beta}(k)=\left(f_{1}(k t), \eta(k) \varphi_{k}^{0}(t)\right)$, $\beta \in I$. В силу (4.19) имеем

$$
\Phi_{\beta}(k)=0 \text { при } k<k_{1}, \quad \frac{1}{\rho(k)} \Phi_{\beta}(k) \underset{k \rightarrow \infty}{\longrightarrow} c^{\beta}, \quad \frac{1}{\rho(k)}\left|\Phi_{\beta}(k)\right|<\text { const },
$$

где const не зависит от $\beta$ и $k$.

Пусть задано $\varepsilon>0$. Докажем, что для любого $\omega \in \mathscr{S}_{\alpha-\varepsilon, 0}^{\alpha+\varepsilon, 0}$ существует $k_{0}$ такое, что

$$
\begin{gathered}
\frac{1}{\rho(k)} \int_{0}^{\infty} \Phi_{\beta}(k \varkappa) \omega(\varkappa) d \varkappa \underset{k \rightarrow+\infty}{\longrightarrow} c_{\omega}^{\beta}, \\
\frac{1}{\rho(k)}\left|\int_{0}^{\infty} \Phi_{\beta}(k \varkappa) \omega(\varkappa) d \varkappa\right| \leqslant \operatorname{const} \mathscr{P}_{\alpha-\varepsilon, 0}^{\alpha+\varepsilon, 0}[\omega], \quad k>k_{0},
\end{gathered}
$$

где const не зависит от $\beta \in I$ и $k>k_{0}$.

Действительно, по лемме 1 для любого $\varepsilon_{1}<\varepsilon$ сушествует $k_{0}$ такое, что выполнено (1.3) (где $\varepsilon$ надо заменить на $\varepsilon_{1}$ ). (Увеличивая, если нужно, $k_{0}$, можно считать, что $k_{1}<k_{0}$.) Имеем

$$
\begin{aligned}
& \frac{1}{\rho(k)} \int_{0}^{\infty} \Phi_{\beta}(k \varkappa) \omega(\varkappa) d \varkappa \\
& \quad=\frac{1}{\rho(k)}\left\{\int_{k_{1} / k}^{k_{0} / k}+\int_{k_{0} / k}^{1-\varepsilon_{1}}+\int_{1-\varepsilon_{1}}^{1+\varepsilon_{1}}+\int_{1+\varepsilon_{1}}^{\infty}\right\} \Phi_{\beta}(k \varkappa) \omega(\varkappa) d \varkappa .
\end{aligned}
$$

Мы учли, что $\eta(k)=0$ при $k<k_{1}$.

Рассмотрим отдельно четыре случая, считая $k<k_{0}$.

1. $k_{1} / k<\varkappa<k_{0} / k$. Пользуясь (4.20), имеем

$$
\begin{aligned}
& \frac{1}{\rho(k)} \int_{k_{1} / k}^{k_{0} / k} \Phi_{\beta}(k \varkappa) \omega(\varkappa) d \varkappa \\
& \leqslant \frac{\text { const }}{\rho(k)} \int_{k_{1} / k}^{k_{0} / k}|\omega(\varkappa)| d \varkappa \leqslant \frac{\text { const }}{\rho(k)} \int_{k_{1} / k}^{k_{0} / k}\left|\varkappa^{\alpha-\varepsilon} \varkappa^{\varepsilon-\alpha} \omega(\varkappa)\right| d \varkappa \\
& \leqslant \frac{\text { const }}{\rho(k)}\left(\frac{k_{3}}{k}\right)^{\varepsilon-\alpha} \int_{0}^{\delta}\left|\varkappa^{\alpha-\varepsilon} \omega(\varkappa)\right| d \varkappa \leqslant \frac{\text { const }}{k^{\varepsilon-\varepsilon_{1}}} \mathscr{P}_{\alpha-\varepsilon, 0}^{\alpha+\varepsilon, 0}[\omega] \underset{k \rightarrow \infty}{\longrightarrow} 0 \text {, }
\end{aligned}
$$

где $k_{3}=k_{0}$, если $\varepsilon-\alpha>0$, и $k_{3}=k_{1}$, если $\varepsilon-\alpha<0$. Здесь учтено также то, что $\rho(k)>$ const $k^{\alpha-\varepsilon_{1}}$ при $k>k_{0}$.

2. $k_{0} / k<\varkappa<1-\varepsilon_{1}$. Имеем

$$
\left|\frac{1}{\rho(k)} \Phi_{\beta}(k \varkappa) \omega(\varkappa)\right| \leqslant \text { const } \frac{\rho(k \varkappa)}{\rho(k)}|\omega(\varkappa)| \leqslant \text { const } \varkappa^{\alpha-\varepsilon_{1}}|\omega(\varkappa)| .
$$

А так как $\int_{0}^{\infty} \varkappa^{\alpha-\varepsilon_{1}}|\omega(\varkappa)| d \varkappa \leqslant$ const $\mathscr{P}_{\alpha-\varepsilon, 0}^{\alpha+\varepsilon, 0}[\omega]$, то по теореме Лебега второй интеграл справа в сумме (4.22) имеет предел при $k \rightarrow+\infty$ и

$$
\frac{1}{\rho(k)}\left|\int_{k_{0} / k}^{1-\varepsilon_{1}} \Phi_{\beta}(k \varkappa) \omega(\varkappa) d \varkappa\right| \leqslant \operatorname{const} \mathscr{P}_{\alpha-\varepsilon, 0}^{\alpha+\varepsilon, 0}[\omega]
$$


3. $1-\varepsilon_{1}<\varkappa<1+\varepsilon_{1}$. Имеем

$$
\left|\frac{1}{\rho(k)} \Phi_{\beta}(k \varkappa) \omega(\varkappa)\right| \leqslant \text { const } \frac{\rho(k \varkappa)}{\rho(k)}|\omega(\varkappa)| \leqslant \text { const }|\omega(\varkappa)| .
$$

А так как $\int_{1-\varepsilon_{1}}^{1+\varepsilon_{1}}|\omega(\varkappa)| d \varkappa \leqslant$ const $\mathscr{P}_{\alpha-\varepsilon, 0}^{\alpha+\varepsilon, 0}[\omega]$, то по теореме Лебега третий интеграл справа в сумме (4.22) имеет предел при $k \rightarrow+\infty$ и

$$
\frac{1}{\rho(k)}\left|\int_{1-\varepsilon_{1}}^{1+\varepsilon_{1}} \Phi_{\beta}(k \varkappa) \omega(\varkappa) d \varkappa\right| \leqslant \operatorname{const} \mathscr{P}_{\alpha-\varepsilon, 0}^{\alpha+\varepsilon, 0}[\omega] .
$$

4. $1+\varepsilon_{1}<\varkappa<\infty$. Имеем

$$
\left|\frac{1}{\rho(k)} \Phi_{\beta}(k \varkappa) \omega(\varkappa)\right| \leqslant \text { const } \frac{\rho(k \varkappa)}{\rho(k)}|\omega(\varkappa)| \leqslant \text { const } \varkappa^{\alpha+\varepsilon_{1}}|\omega(\varkappa)| .
$$

А так как

$$
\int_{1+\varepsilon_{1}}^{\infty} \varkappa^{\alpha+\varepsilon_{1}}|\omega(\varkappa)| d \varkappa \leqslant \int_{1+\varepsilon_{1}}^{\infty} \varkappa^{\alpha+\varepsilon}|\omega(\varkappa)| d \varkappa \leqslant \text { const } \mathscr{P}_{\alpha-\varepsilon, 0}^{\alpha+\varepsilon, 0}[\omega],
$$

то по теореме Лебега последний интеграл справа в сумме (4.22) имеет предел при $k \rightarrow+\infty$ и

$$
\frac{1}{\rho(k)}\left|\int_{1+\varepsilon_{1}}^{\infty} \Phi_{\beta}(k \varkappa) \omega(\varkappa) d \varkappa\right| \leqslant \text { const } \mathscr{P}_{\alpha-\varepsilon, 0}^{\alpha+\varepsilon, 0}[\omega] .
$$

Из вышесказанного вытекает, что интеграл (4.22) имеет предел при $k \rightarrow+\infty$, а из оценок (4.23)-(4.26) вытекает оценка (4.21).

Теперь нам осталось показать, что для любого $\omega \in \mathscr{S}_{0}^{a, 0} \alpha-\varepsilon, 0$, любого фиксированного $k$ и $\beta \in I$

$$
\int_{0}^{\infty} \Phi_{\beta}(k \varkappa) \omega(\varkappa) d \varkappa=\left(f_{1}(k t),\left(B_{k}^{\beta} \omega\right)(t)\right) .
$$

Легко видеть, что это достаточно сделать только в случае, когда $\omega$ - бесконечно дифференцируемая функция с компактным носителем в $(0,+\infty)$. Действительно, с одной стороны, множество таких функций плотно в $\mathscr{S}_{\alpha-\varepsilon, 0}^{a, 0}$, а с другой сторо-

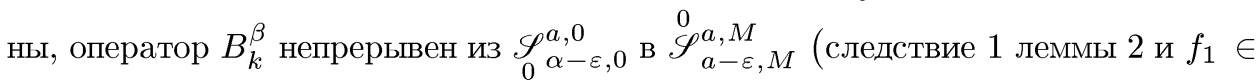
$\left.\left(\mathscr{S}_{\alpha-\varepsilon, M}^{a, M}\right)^{\prime}\right)$. В этом случае отображение $\varkappa \mapsto \psi_{\varkappa}=\eta(k \varkappa) \omega(\varkappa)(1 / \varkappa)_{\varphi}^{0} \beta \varkappa(t / \varkappa)$ есть непрерывное отображение отрезка (носителя функции $\omega(\varkappa))$ в $\stackrel{\mathscr{S}}{b, M}_{b, M}^{a} \subset$ $\stackrel{\mathscr{S}}{a}_{\alpha-\varepsilon, M}^{a, M}$. Воспользуемся теперь следующим общим фактом: если отображение $\varkappa \mapsto \psi_{\varkappa}$ есть непрерьвное отображение отрезка $[c, d]$ в банахово пространство $\mathbb{B}$, a $f_{1} \in \mathbb{B}^{\prime}$, то $\left(f_{1}, \int_{c}^{d} \psi_{\varkappa} d \varkappa\right)=\int_{c}^{d}\left(f_{1}, \psi_{\varkappa}\right) d \varkappa$, где интеграл (слева) понимается как интеграл Фреше. Имеем

$$
\begin{aligned}
\left(f_{1}(k t),\left(B_{k}^{\beta} \omega\right)(t)\right) & =\left(f_{1}(k t), \int_{0}^{\infty} \varphi^{0} \beta\left(\frac{t}{\varkappa}\right) \eta(k \varkappa) \omega(\varkappa) \frac{d \varkappa}{\varkappa}\right) \\
& =\int_{0}^{\infty}\left(f_{1}(k t), \stackrel{0}{\varphi}_{k \varkappa}^{\beta}\left(\frac{t}{\varkappa}\right) \eta(k \varkappa) \omega(\varkappa) \frac{1}{\varkappa}\right) d \varkappa \\
& =\int_{0}^{\infty}\left(f_{1}(k t \varkappa), \eta(k \varkappa) \stackrel{0}{\varphi}_{k \varkappa}^{\beta}(t)\right) \omega(\varkappa) d \varkappa=\int_{0}^{\infty} \Phi_{\beta}(k \varkappa) \omega(\varkappa) d \varkappa .
\end{aligned}
$$

Лемма доказана. 
СлеДСТВИЕ. Пусть семейство $\left\{\omega^{\beta}(t) \in \mathscr{S}_{\alpha-\varepsilon, 0}^{a, 0}, \quad \beta \in I\right\}=\omega(t)$ принадле-

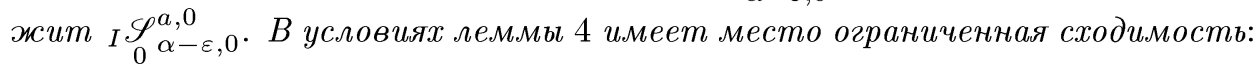

$$
\frac{1}{\rho(k)}\left(f_{1}(k t), B_{k} \omega(t)\right) \underset{k \rightarrow+\infty}{\longrightarrow} c_{\omega},
$$

где оператор $B_{k}$ определен формулой (3.16), при этом

$$
\frac{1}{\rho(k)}\left|\left(f_{1}(k t), B_{k} \omega(t)\right)\right| \leqslant A \cdot{ }_{I} \mathscr{P}_{\alpha-\varepsilon, 0}^{\alpha+\varepsilon, 0}[\omega]
$$

где $A$ не зависит от $k>k_{0}$.

ДоКАЗАТЕЛЬСтво. Сначала заметим, что семейство последовательностей $\left\{\eta(k) \varphi_{k}^{\beta}(t), \beta \in I\right\}$ принадлежит ${ }^{I} F^{0}\left[\mathscr{S}_{\alpha-\varepsilon, M}^{a, M}\right]$. Поэтому в силу следствия 2 леммы 2 оператор $B_{k}:{ }_{I} \mathscr{S}_{\alpha-\varepsilon, 0}^{a, 0} \longrightarrow \stackrel{0}{\mathscr{S}_{\alpha-\varepsilon, M}^{a, M}}$ корректно определен; в частности, ряд $\sum_{\beta \in I} B_{k}^{\beta} \omega_{\beta}(t)$ сходится в $\stackrel{0}{\mathscr{S}}_{\alpha-\varepsilon, M}^{a, M}$. Так как $f_{1} \in\left(\mathscr{S}_{\alpha-\varepsilon, M}^{a, M}\right)^{\prime}$, то при $k>k_{0}$, пользуясь (4.17), имеем

$$
\begin{aligned}
& \frac{1}{\rho(k)}\left|\left(f_{1}(k t), B_{k} \omega(t)\right)\right| \leqslant \frac{1}{\rho(k)}\left|\left(f_{1}(k t), \sum_{\beta \in I} B_{k}^{\beta} \omega^{\beta}(t)\right)\right| \\
& \leqslant \frac{1}{\rho(k)} \sum_{\beta \in I}\left|\left(f_{1}(k t), B_{k}^{\beta} \omega^{\beta}(t)\right)\right| \leqslant A \sum_{\beta \in I} \mathscr{P}_{\alpha-\varepsilon, 0}^{\alpha+\varepsilon, 0}\left[\omega^{\beta}\right]=A \cdot{ }_{I} \mathscr{P}_{\alpha-\varepsilon, 0}^{\alpha+\varepsilon, 0}[\omega],
\end{aligned}
$$

что доказывает (4.30).

Из (4.16) и того факта, что ряд $\frac{1}{\rho(k)} \sum_{\beta \in I}\left(f_{1}(k t), B_{k}^{\beta} \omega^{\beta}(t)\right)$ мажорируется сходяшимся рядом с членами, не зависяшими от $k$, следует (4.29). Следствие доказано.

ЛЕмма 5. Пусть для $f(t) \in\left(\mathscr{S}_{b, N, \eta}^{a, M}\right)^{\prime}$ и семейства последовательностей функций $\left\{\varphi_{\lambda}^{\beta}(t) \in \mathscr{S}_{b, N, \delta}^{a, M}, \quad \beta \in I, \lambda>0\right\}$ выполнень условия леммы 4. Пусть также для некоторой функиии $\varphi(t) \in \mathscr{S}_{b, N, \delta}^{a, M}$ существуют $k^{*}>0, \varepsilon>0 u$ семейство последовательностей

$$
\omega_{k}(t) \in{ }_{I} \mathscr{S}_{0}^{a, 0} \alpha-\varepsilon, 0, \quad \omega_{k}(t)=\left\{\omega_{k}^{\beta}(t) \in \mathscr{S}_{0}^{a, 0} \alpha-\varepsilon, 0, \beta \in I\right\}, \quad k>k^{*},
$$

такие, что

$$
\begin{gathered}
\omega_{k}(t) \underset{k \rightarrow+\infty}{\longrightarrow} \omega(t) \quad \theta_{I} \mathscr{S}_{0}^{a, 0} \alpha, 0, \\
\mathscr{P}_{a, M}^{a, M}\left[\varphi^{0}-B_{k} \omega_{k}\right] \leqslant \frac{C}{k^{a-\alpha+\varepsilon}}, \quad k>k^{*},
\end{gathered}
$$

c некоторой постоянной $C$, где $\stackrel{0}{\varphi}(t)=\varphi(t)-T_{\varphi}^{\langle a\rangle}(t)$. Здесь семейство операторов $B_{k}$ построено по семейству последовательностей $\eta(\lambda) \varphi_{\lambda}(t)$, как в лемме 4.

Тогда $f(t)$ обладает квазиасимптотикой относительно $\rho(k)$ на $\varphi$, т.е.

$$
\frac{1}{\rho(k)}(f(k t), \varphi(t)) \longrightarrow \text { const } \quad \text { npu } \quad k \rightarrow+\infty .
$$


ДоКАЗАТЕЛЬСТВо. По лемме 4 для любого $Q\rangle\langle b\rangle$ функционал $f(t)$ представляется в виде $f(t)=f_{1}(t)+f_{2}(t)$, причем $f_{1} \in\left(\mathscr{S}^{a, M}\right)^{\prime}$ и $\left(f_{1}(t), t^{j}\right)=0$ для $j=0,1, \ldots,\langle a\rangle$, а $f_{2} \in\left(\mathscr{S}_{b, N}\right)^{\prime}$ и $\left(f_{2}(t), t^{\ell}\right)=0$ для $\ell=0,1, \ldots, Q$. Так как $f_{2} \in\left(\mathscr{S}_{b, N, \delta_{1}}^{b+\varepsilon, M}\right)^{\prime}$ с некоторым $\delta_{1}>0$ и все ее моменты до порядка $\langle b\rangle$ обращаются в нуль, то по лемме 3 имеем

$$
\frac{1}{\rho(k)}\left|\left(f_{2}(k t), \varphi(t)\right)\right| \leqslant \text { const } \frac{k^{b+\varepsilon}}{\rho(k)} \longrightarrow 0 \text { при } k \rightarrow+\infty
$$

ибо порядок автомодельности $\rho(k)$ равен $\alpha>b+\varepsilon$. А потому

$$
\begin{aligned}
\frac{1}{\rho(k)}(f(k t), \varphi(t)) & =\frac{1}{\rho(k)}\left(f_{1}(k t), \varphi(t)\right)+\frac{1}{\rho(k)}\left(f_{2}(k t), \varphi(t)\right) \\
& =\frac{1}{\rho(k)}\left(f_{1}(k t), \stackrel{0}{\varphi}(t)\right)+o(1) \text { при } k \rightarrow+\infty,
\end{aligned}
$$

и (4.33) будет выполнено, если мы покажем, что $\frac{1}{\rho(k)}\left(f_{1}(k t), \stackrel{0}{\varphi}(t)\right) \rightarrow$ const при $k \rightarrow+\infty$.

Согласно следствию к лемме 4 существует $\lim _{k \rightarrow \infty} \frac{1}{\rho(k)}\left(f_{1}(k t), B_{k} \omega\right)$. Обозначая $B_{k} \omega-\stackrel{0}{\varphi}$ через $\gamma_{k}(t) \in \stackrel{0}{\mathscr{S}_{b, M}^{a, M}}$, учитывая условие (4.32) и тот факт, что $\mathscr{P}_{a, M}^{a, M}\left[\gamma_{k}(t / k)\right]=k^{a+1} \mathscr{P}_{a, M}^{a, M}\left[\gamma_{k}(t)\right]$, при достаточно больших $k$ имеем

$$
\begin{aligned}
& \frac{1}{\rho(k)}\left|\left(f_{1}(k t), B_{k} \omega-\stackrel{0}{\varphi}\right)\right|=\frac{1}{k \rho(k)}\left|\left(f_{1}(t), \gamma_{k}\left(\frac{t}{k}\right)\right)\right| \\
& \quad \leqslant \frac{1}{k \rho(k)} \operatorname{const} \mathscr{P}_{a, M}^{a, M}\left[\gamma_{k}\left(\frac{t}{k}\right)\right] \leqslant \operatorname{const} \frac{k^{a}}{\rho(k)} \mathscr{P}_{a, M}^{a, M}\left[\gamma_{k}(t)\right] \\
& \leqslant \operatorname{const} \frac{k^{a}}{\rho(k)} \frac{1}{k^{a-\alpha+\varepsilon}} \leqslant \operatorname{const} \frac{k^{\alpha-\varepsilon}}{\rho(k)} \rightarrow 0, \quad k \rightarrow+\infty
\end{aligned}
$$

Далее имеем

$$
\frac{1}{\rho(k)}\left(f_{1}(k t), \stackrel{0}{\varphi}(t)\right)=\frac{1}{\rho(k)}\left(f_{1}(k t), B_{k} \omega_{k}\right)-\frac{1}{\rho(k)}\left(f_{1}(k t), B_{k} \omega_{k}-\stackrel{0}{\varphi}\right) .
$$

Вьше мы показали, что оба слагаемых в правой части имеют предел. Лемма доказана.

ЗАмЕчАниЕ. В случае $\varphi_{k}^{\beta}(t) \equiv \varphi_{0}^{\beta}(t), \beta \in I$, условие (4.13) можно заменить на следующее условие: если $\left(\varphi_{0}^{\beta}\right)^{(j)}(0)=0$ для некоторого $j$ из $\{0,1, \ldots,\langle a\rangle\}$ и всех $\beta \in I$, то $(\varphi)^{(j)}(0)=0$ для этого $j$.

Условия (4.31) и (4.32) можно интерпретировать в некотором смысле как почти обратимость справа семейства линейных операторов $B_{k}$ при $k \rightarrow+\infty$. Оператор $B_{0}$, введенный в предыдушем параграфе (см. (3.3)), является в некотором смысле предельным оператором для этого семейства. Для дальнейшего важно иметь достаточные условия существования правого обратного оператора $B_{0}^{-1}$. Следуюшая лемма в какой-то степени решает эту задачу. 
ЛЕмма 6. Пусть I-счетное множсество и семейство функиий

$$
\left\{\varphi_{0}^{\beta}(t) \in \mathscr{S}_{b, N, \delta}^{a, M}, \beta \in I\right\}=\varphi_{0}(t), \quad b<a, \quad M \leqslant N
$$

удовлетворяет условиям:

1) $\varphi_{0}(t) \in{ }^{I} \mathscr{S}_{b, N, \delta}^{a, M}$, m.e. $\sup _{\beta \in I} \mathscr{P}_{b, N, \delta}^{a, M}\left[\varphi_{0}^{\beta}(t)\right]<\infty$;

2) существуют $A$ и т такие, что

$$
\sup _{\beta \in I}\left|\widehat{\varphi}_{0}^{\beta}(z)\right| \geqslant \frac{A}{1+|z|^{m}}, \quad z \in \Pi_{b}^{a},
$$

где $\widehat{\varphi}_{0}^{\beta}(z)=M\left[\varphi_{0}^{\beta}(t)\right]$ - преобразование Меллина, определяемое формулой (2.9);

3) для любого $j=0,1, \ldots,\langle b\rangle$ существуют $\beta_{j} \in I$ такие, что

$$
\left(\frac{d}{d t}\right)^{j} \varphi_{0}^{\beta_{j}}(0) \neq 0, \quad j=0,1, \ldots,\langle b\rangle .
$$

Тогда для любих $\varepsilon>0(a-\varepsilon>b+\varepsilon)$ и $M_{1} \geqslant 0$ найдется число $Q$ такое, что для любого $n \geqslant Q$ существует непрерывный правый обратный оператор

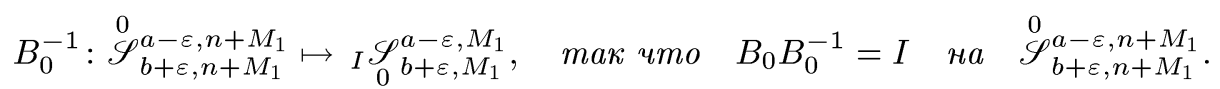

ДокаЗАТЕльство. Пусть задано $\varepsilon>0$ и $M_{1} \geqslant 0$. Согласно лемме П0 и замечаниям 1 и 2 к ней существуют числа $\lambda_{\beta}>0, \beta \in I, \sum_{\beta \in I} \lambda_{\beta}<\infty$, такие, что для некоторых постоянных $A_{1}$ и $m_{1}$

$$
\sum_{\beta \in I} \lambda_{\beta}\left|\hat{\varphi}_{0}^{\beta}\right|^{2} \geqslant \frac{A_{1}}{(1+|z|)^{m_{1}}}, \quad z \in \Pi_{b+\varepsilon / 4}^{a-\varepsilon / 4} .
$$

А тогда согласно лемме П1 существуют число $Q_{1}$ и семейство аналитических в $\Pi_{b+\varepsilon / 2}^{a-\varepsilon / 2}$ функций $\left\{g_{\beta}(z) \in H_{Q_{1}}\left(\Pi_{b+\varepsilon / 2}^{a-\varepsilon / 2}\right), \beta \in I\right\}$ такие, что

$$
\begin{gathered}
\sup _{\beta \in I}\left\|g_{\beta}(z)\right\|_{H_{Q_{1}}}<\infty, \quad \sum_{\beta \in I} \lambda_{\beta} g_{\beta}(z) \widehat{\varphi}_{0}^{\beta}(z)=1, \\
g_{\beta}\left(z_{\ell}\right)=0 \quad \forall \beta \in I, \quad z_{\ell}=-i \ell, \quad \ell=1,2, \ldots, \quad z_{\ell} \in \Pi_{b}^{a} .
\end{gathered}
$$

Определим (пока формально) оператор $B_{0}^{-1}$ формулой

$$
\left(B_{0}^{-1} \varphi\right)^{\beta}(t)=M^{-1}\left[\lambda_{\beta} \widehat{\varphi}(z) g_{\beta}(z)\right], \quad \varphi(t) \in \stackrel{0}{\mathscr{S}}_{b+\varepsilon, n+M_{1}}^{a-\varepsilon, n+M_{1}}
$$

где $M^{-1}$ - обратное преобразование Меллина (см. (2.13)).

Докажем, что если $n \geqslant-Q_{1}+2$, то $B_{0}^{-1}$ корректно определен как непрерьвный оператор из $\stackrel{0}{\mathscr{S}}_{b+\varepsilon, n+M_{1}}^{a-\varepsilon, n+M_{1}}$ в ${ }_{i} \stackrel{0}{\mathscr{S}}{ }_{b+\varepsilon, M_{1}}^{a-\varepsilon, M_{1}}$. Действительно, пусть $P(-i z)$ - полином степени $-Q_{1}+2$, не имеющий корней в полосе $\Pi_{b}^{a}$ (если $Q_{1} \geqslant 2$, то считаем $P(-i z) \equiv 1)$. Обозначим $\widehat{G}_{\beta}(z)=\frac{g_{\beta}(z)}{P(-i z)}$. Так как $\left|\widehat{G}_{\beta}(z)\right|<\frac{\text { const }}{1+|z|^{2}}, z \in \Pi_{b+\varepsilon / 2}^{a-\varepsilon / 2}$ 
(заметим, что const не зависит от $\beta \in I$ ), то в силу (2.14) $G_{\beta}(t)=M^{-1}\left[\widehat{G}_{\beta}(z)\right] \in$ $\mathscr{S}_{0}^{a-\varepsilon, 0} b$. (Мы поставили здесь 0 снизу в силу того, что $\widehat{G}_{\beta}(z)$ не имеет полюсов в $\left.\Pi_{b+\varepsilon / 2}^{a-\varepsilon / 2}.\right)$ Более того, семейство $\left\{G_{\beta}(t), \beta \in I\right\}$ принадлежит ${ }_{0}^{I} \mathscr{S}_{b+\varepsilon, 0}^{a-\varepsilon, 0}$. Имеем (считая $-Q_{1}+2 \geqslant 0$ )

$$
\begin{aligned}
\mathscr{P}_{b+\varepsilon, M_{1}}^{a-\varepsilon, M_{1}}\left[\left(B_{0}^{-1} \varphi\right)^{\beta}\right] & =\lambda_{\beta} \mathscr{P}_{b+\varepsilon, M_{1}}^{a-\varepsilon, M_{1}}\left[M^{-1}\left[\frac{g_{\beta}(z)}{P(-i z)} \cdot P(-i z) \widehat{\varphi}(z)\right]\right] \\
& =\lambda_{\beta} \mathscr{P}_{b+\varepsilon, M_{1}}^{a-\varepsilon, M_{1}}\left[P\left(t \frac{d}{d t}\right) \varphi(t) * G_{\beta}(t)\right] \\
& \leqslant \operatorname{const} \lambda_{\beta} \mathscr{P}_{b+\varepsilon, M_{1}}^{a-\varepsilon, M_{1}}\left[P\left(t \frac{d}{d t}\right) \varphi\right] \cdot \mathscr{P}_{b+\varepsilon, 0}^{a-\varepsilon, 0}\left[G_{\beta}(t)\right] \\
& \leqslant \operatorname{const} \lambda_{\beta} \mathscr{P}_{b+\varepsilon, 0}^{a-\varepsilon, 0}\left[G_{\beta}(t)\right] \cdot \mathscr{P}_{b+\varepsilon,-Q_{1}+2+M_{1}}^{a-\varepsilon,-Q_{1}+2+M_{1}}[\varphi] .
\end{aligned}
$$

Мы воспользовались утверждением 1 (см. $\S 2$, а также лемму П2). Суммируя теперь (4.42) по $\beta \in I$, получим

$$
{ }_{I} \mathscr{P}_{b+\varepsilon, M_{1}}^{a-\varepsilon, M_{1}}\left[B_{0}^{-1} \varphi\right] \leqslant \mathrm{const} \cdot{ }^{I} \mathscr{P}_{b+\varepsilon, 0}^{a-\varepsilon, 0}\left[G_{\beta}(t)\right] \cdot \mathscr{P}_{b+\varepsilon,-Q_{1}+2+M_{1}}^{a-\varepsilon,-Q_{1}+2+M_{1}}[\varphi],
$$

что и требовалось проверить.

Из (4.40) следует, что $\widehat{\varphi}(z) g_{\beta}(z)$ не имеют полюсов в $\Pi_{b+\varepsilon}^{a-\varepsilon}$, поэтому $B_{0}^{-1} \varphi \in$ ${ }_{I} \mathscr{S}_{0}^{a-\varepsilon, M_{1}}$. Осталось проверить, что $B_{0} B_{0}^{-1}=I$. Для $\varphi(t) \in \mathscr{S}_{0}^{a-\varepsilon, n+M_{1}}$, взяв преобразование Меллина от $B_{0} B_{0}^{-1} \varphi$ и учитывая (4.39), получим

$$
\begin{aligned}
M\left[B_{0} B_{0}^{-1} \varphi\right] & =M\left[\sum_{\beta \in I}\left(B_{0}^{-1} \varphi\right)^{\beta} * \stackrel{0}{\varphi}_{0}^{\beta}\right]=M\left[\sum_{\beta \in I} M^{-1}\left[\lambda_{\beta} \widehat{\varphi}(z) g_{\beta}(z)\right] * 0_{0}^{0}\right] \\
& =\sum_{\beta \in I} \lambda_{\beta} g_{\beta}(z) \widehat{\varphi}(z) \widehat{\varphi}_{0}^{\beta}(z)=\widehat{\varphi}(z) \sum_{\beta \in I} \lambda_{\beta} g_{\beta}(z) \widehat{\varphi}_{0}^{\beta}(z)=\widehat{\varphi}(z) .
\end{aligned}
$$

Отсюда, учитывая, что $\varphi(t) \in \stackrel{0}{\mathscr{S}}_{b+\varepsilon, n+M_{1}}^{a-\varepsilon, n+M_{1}}$, выводим, что $\varphi(t)=\left(B_{0} B_{0}^{-1} \varphi\right)(t)$. Лемма доказана.

\section{§ 5. Основные теоремы}

Tеорема 1. Пусть $f(t) \in\left(\mathscr{S}_{b, N, \eta}^{a, M}\right)^{\prime}, b<a, M \leqslant N, \rho(k)$ - автомодельная функиия порядка $\alpha, b<\alpha<a$, и задано семейство последовательностей функиий

$$
\left\{\varphi_{\lambda}^{\beta}(t) \in \mathscr{S}_{b, N, \delta}^{a+\varepsilon_{0}, M}, \quad \beta \in I, \quad \lambda>\lambda_{0}\right\} \equiv \varphi_{\lambda}(t)
$$

для некоторых $\lambda_{0}>0$ и $\varepsilon_{0}>0$ со свойствами:

1) $\varphi_{\lambda}^{\beta}(t)$ непрерывна по $\lambda$ в $\mathscr{S}_{b, N, \delta}^{a+\varepsilon_{0}, M}$, при этом

$$
\mathscr{P}_{b, N, \delta}^{a+\varepsilon_{0}, M}\left[\varphi_{\lambda}^{\beta}(t)\right]<\mathrm{const},
$$

где const не зависит от $\beta \in I$ и $\lambda>\lambda_{0}$; 
2) $\forall \beta \in I \quad \varphi_{\lambda}^{\beta}(t) \underset{\lambda \rightarrow+\infty}{\longrightarrow} \varphi_{0}^{\beta}(t)$ в $\mathscr{S}_{b, N, \delta}^{a+\varepsilon_{0}, M}$;

3) $\varphi_{\lambda}^{\beta}(t)$ бесконечно дифференцируема по $\lambda$ (в смысле Фреше) в $\mathscr{S}_{\alpha-\varepsilon, M}^{a, M}$ для некоторого $\varepsilon>0(\alpha-\varepsilon>b)$, причем существует $\sigma>0$ такое, что

$$
\mathscr{P}_{\alpha-\varepsilon, M}^{a, M}\left[\left(\lambda \frac{d}{d \lambda}\right)^{p}\left(\stackrel{0}{\varphi}_{\lambda}^{\beta}(t)-\stackrel{0}{\varphi}_{0}^{\beta}(t)\right)\right] \leqslant \frac{c_{p}}{\lambda^{\sigma}}, \quad p=0,1, \ldots,
$$

где с с не зависит от $\lambda>\lambda_{0} u \beta \in I$. Здесь $\stackrel{0}{\varphi}_{\lambda}^{\beta}(t)$ u $\stackrel{0}{\varphi}_{0}^{\beta}(t)$ определяются согласно (2.6).

Пусть еще семейство $\varphi_{0}(t)=\left\{\varphi_{0}^{\beta}(t), \beta \in I\right\}$ удовлетворяет следующим условиям:

а) существуют $A>0$ и $m \geqslant 0$ такие, что

$$
\sup _{\beta \in I}\left|\widehat{\varphi}_{0}^{\beta}(z)\right| \geqslant \frac{A}{1+|z|^{m}} \quad \text { npu } \quad z \in \Pi_{\alpha-\varepsilon}^{a+\varepsilon_{0}}
$$

(здесь $\widehat{\varphi}_{0}^{\beta}(z)$ - преобразование Меллина $\varphi_{0}^{\beta}(t)(c \mathcal{M} .(2.9))$;

б) для любого $j=0,1, \ldots,\langle\alpha-\varepsilon\rangle$ найдутся $\beta_{j} \in I$ такие, что

$$
\frac{d^{j}}{d t^{j}} \varphi_{0}^{\beta_{j}}(0) \neq 0 \quad \partial \Omega я \quad j=0,1, \ldots,\langle\alpha-\varepsilon\rangle .
$$

Тогда если обобщенная функция $f(t)$ обладает ограниченной по $\beta \in I$ квазиасимптотикой относительно $\rho(k)$ на семействе последовательностей $\varphi_{\lambda}(t)$, m.e.

$$
\frac{1}{\rho(k)}\left(f(k t), \varphi_{k}^{\beta}(t)\right) \underset{k \rightarrow+\infty}{\longrightarrow} c^{\beta} \quad \forall \beta \in I,
$$

причем существует постоянная $c$, не зависящая от $\beta \in I$, такая, что

$$
\frac{1}{\rho(k)}\left|\left(f(k t), \varphi_{k}^{\beta}(t)\right)\right| \leqslant c,
$$

то $f(t)$ обладает квазиасимптотикой относительно $\rho(k)$, m.е.

$$
\frac{1}{\rho(k)}(f(k t), \varphi(t)) \underset{k \rightarrow+\infty}{\longrightarrow} c_{\varphi} \quad \forall \varphi(t) \in \mathscr{S}_{+} .
$$

ДоКАЗАТЕЛЬСТВо. Для того чтобы доказать теорему, мы воспользуемся леммой 5 . Однако для этого необходимо сделать несколько предварительных построений. Пусть $\eta(\lambda)-C^{\infty}$-функция такая, что $\eta(\lambda)=0$ при $\lambda<\lambda_{0}$ и $\eta(\lambda) \equiv 1$ при $\lambda>\lambda_{1}>\lambda_{0}$. Тогда в силу (5.1) и (5.2)

$$
\eta(\lambda) \varphi_{\lambda}(t) \in{ }^{I} F^{0}\left[\mathscr{S}_{b, N, \delta}^{a+\varepsilon_{0}, M}\right] .
$$

Аналогично, в силу (5.4)

$$
\lambda^{\sigma} \eta(\lambda)\left(\stackrel{0}{\varphi}_{\lambda}(t)-\stackrel{0}{\varphi}_{0}(t)\right) \in{ }^{I} F^{\infty}\left[\stackrel{0}{\mathscr{S}_{\alpha-\varepsilon, M}^{a, M}}\right] .
$$


Из (5.2) и (5.3) следует, что

$$
\left\{\varphi_{0}^{\beta}(t) \in \mathscr{S}_{b, N, \delta}^{a+\varepsilon_{0}, M}, \beta \in I\right\}=\varphi_{0}(t) \in{ }^{I} \mathscr{S}_{b, N, \delta}^{a+\varepsilon_{0}, M} .
$$

Поэтому для любого $M_{1} \geqslant 0$ определен линейный непрерывный оператор

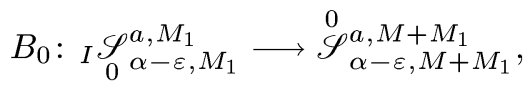

действуюший по формуле (3.3). Из (5.12), (5.5) и (5.6) следует, что выполнены все условия леммы 6 об обратном операторе. А потому сушествует такое $n$, что для любого $M_{1} \geqslant 0$ определен непрерывный правый обратный оператор

$$
B_{0}^{-1}: \stackrel{0}{\mathscr{S}}_{\alpha-\varepsilon, n+M_{1}}^{a, n+M_{1}} \longrightarrow{ }_{I} \mathscr{S}_{0}^{a, M_{1}}{ }_{\alpha, M_{1}}
$$

(где, быть может, следует немного уменьшить $\varepsilon$ ) такой, что $B_{0} B_{0}^{-1}=I$.

В силу (5.10) (см. следствие 1 к лемме 2) последовательность $\eta(\lambda) \varphi_{\lambda}(t)$ определяет семейство линейных непрерывных операторов

$$
B_{k}:{ }_{I} \mathscr{S}_{\alpha-\varepsilon, 0}^{a, 0} \longrightarrow \stackrel{0}{\mathscr{S}}_{\alpha-\varepsilon, M}^{a, M}, \quad k>0
$$

действуюших по формулам (3.16), (3.14). Учитывая (5.11), нетрудно проверить, что

$$
\stackrel{0}{\varphi}_{0}(t)-\eta(\lambda) \stackrel{\varphi}{\varphi}_{\lambda}(t) \equiv v_{\lambda}(t) \in{ }^{I} F^{\infty}\left[\begin{array}{l}
0 \\
\mathscr{S}_{\alpha-\varepsilon, M}^{a, M}
\end{array}\right]
$$

Поэтому для любого $M_{1} \geqslant 0$ семейство последовательностей $v_{\lambda}(t)=\left\{v_{\lambda}^{\beta}(t)\right.$, $\beta \in I, \lambda>0\}$ определяет семейство линейных непрерывных операторов (см. (3.16), $(3.14))$

$$
R_{k}:{ }_{I} \mathscr{S}_{0}^{a, M_{1}} \underset{\alpha-\varepsilon, M_{1}}{\longrightarrow} \stackrel{0}{\mathscr{S}}_{\alpha-\varepsilon, M+M_{1}}^{a, M+M_{1}} .
$$

Очевидно, что $R_{k}=B_{0}-B_{k}$. По лемме П4 для любого $d \in(b, a)$ такого, что $d-\sigma>\alpha-\varepsilon$, имеем

$$
\mathscr{P}_{d, M+M_{1}}^{a, M+M_{1}}\left[R_{k} \omega\right] \leqslant \frac{\mathrm{const}}{k^{\sigma}} \cdot{ }^{I} \mathscr{P}_{(d, M)}^{M_{1}(a, M)}\left[\lambda^{\sigma} v_{\lambda}\right] \cdot{ }_{I} \mathscr{P}_{d-\sigma, M_{1}}^{a, M_{1}}[\omega]
$$

Теперь все готово, чтобы воспользоваться леммой 5 . Пусть фиксирована функция $\varphi(t) \in \mathscr{S}_{b, Q}^{a, Q}$, где $Q$ достаточно велико и будет выбрано позднее. Подберем семейство последовательностей функций $\omega_{k} \in{ }_{I}^{\mathscr{S}_{0}^{a}} \alpha^{a, 0}$, условия (4.31) и (4.32) леммы 5. Для этого положим

$$
\omega_{k}=B_{0}^{-1} \sum_{j=0}^{L}\left(R_{k} B_{0}^{-1}\right)^{j} \stackrel{0}{\varphi}
$$


где $\stackrel{0}{\varphi}(t)=\varphi(t)-T_{\varphi}^{\langle a\rangle}(t) \in \stackrel{0}{\mathscr{S}}_{\alpha-\varepsilon, Q}^{a, Q}$, а $L$ подберем позднее. Из (5.14) и (5.17), считая $M_{1}=Q-n$, имеем

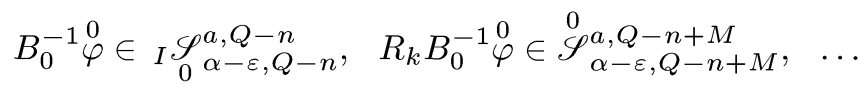

$$
\begin{aligned}
& \ldots, \quad\left(R_{k} B_{0}^{-1}\right)^{j} \stackrel{0}{\varphi} \in \stackrel{\mathscr{S}}{\alpha-\varepsilon, Q-j(n-M)}_{\alpha, Q-j(n-M)}^{a}, \ldots \\
& \ldots, \quad\left(R_{k} B_{0}^{-1}\right)^{L} \stackrel{0}{\varphi} \in \stackrel{0}{\mathscr{S}}_{\alpha-\varepsilon, Q-L(n-M)}^{a, Q-L(n-M)}, \\
& \omega_{k} \in I_{0}^{\mathscr{J}^{a, Q-\varepsilon}, Q-(L+1)(n-M)-M}
\end{aligned}
$$

Если $Q$ достаточно велико, то $\omega_{k}$ - хорошо определенное семейство последовательностей функций. Пользуясь тем, что $B_{0}^{-1}-$ правый обратный к $B_{0}$ оператор, имеем

$$
\stackrel{0}{\varphi}-B_{k} \omega_{k}=\stackrel{0}{\varphi}-\left(B_{0}-R_{k}\right) B_{0}^{-1} \sum_{j=0}^{L}\left(R_{k} B_{0}^{-1}\right)^{j} \stackrel{0}{\varphi}=\left(R_{k} B_{0}^{-1}\right)^{L+1} \stackrel{0}{\varphi}
$$

Учитывая $(5.14),(5.18)$ и $(5.21)$ и обозначая $n-M$ через $\Delta$, имеем

$$
\begin{aligned}
& \mathscr{P}_{a, M}^{a, M}\left[\stackrel{0}{\varphi}-B_{k} \omega_{k}\right]=\mathscr{P}_{a, M}^{a, M}[\underbrace{\left(R_{k} B_{0}^{-1}\right) \ldots\left(R_{k} B_{0}^{-1}\right)}_{L+1}{ }^{0}] \\
& \leqslant \frac{\text { const } I}{k^{\sigma}} \mathscr{P}_{(a, M)}^{0(a, M)}\left[\lambda^{\sigma} v_{\lambda}\right]_{I} \mathscr{P}_{a-\sigma, 0}^{a, 0}[B_{0}^{-1} \underbrace{\left(R_{k} B_{0}^{-1}\right) \ldots\left(R_{k} B_{0}^{-1}\right)}_{L}{ }^{0}] \\
& \leqslant \frac{\text { const } I}{k^{\sigma}} \mathscr{P}_{(a, M)}^{0(a, M)}\left[\lambda^{\sigma} v_{\lambda}\right] \cdot \mathscr{P}_{a-\sigma, n}^{a, n}[\underbrace{\left(R_{k} B_{0}^{-1}\right) \ldots\left(R_{k} B_{0}^{-1}\right)}_{L}{ }^{0}] \\
& \leqslant \frac{\text { const }}{k^{2 \sigma}} \mathscr{P}_{(a, M)}^{0(a, M)}\left[\lambda^{\sigma} v_{\lambda}\right]{ }^{I} \mathscr{P}_{(a-\sigma, M)}^{\Delta(a, M)}\left[\lambda^{\sigma} v_{\lambda}\right] \\
& \times{ }_{I} \mathscr{P}_{a-2 \sigma, \Delta}^{a, \Delta}[B_{0}^{-1} \underbrace{\left(R_{k} B_{0}^{-1}\right) \ldots\left(R_{k} B_{0}^{-1}\right)}_{L-1} \stackrel{0}{\varphi}] \\
& \leqslant \frac{\text { const }}{k^{2 \sigma}}\left\{\mathscr{P}_{(a, M)}^{0(a, M)}\left[\lambda^{\sigma} v_{\lambda}\right] \mathscr{P}_{(a-\sigma, M)}^{\Delta(a, M)}\left[\lambda^{\sigma} v_{\lambda}\right]\right\} \\
& \times \mathscr{P}_{a-2 \sigma, 2 \Delta+M}^{a, 2 \Delta+M}[\underbrace{\left(R_{k} B_{0}^{-1}\right) \ldots\left(R_{k} B_{0}^{-1}\right)}_{L-1}{ }^{0} \varphi \leqslant \cdots \\
& \cdots \leqslant \frac{\text { const }}{k^{(L+1) \sigma}}\left\{I \mathscr{P}_{(a, M)}^{0(a, M)}\left[\lambda^{\sigma} v_{\lambda}\right]{ }^{I} \mathscr{P}_{(a-\sigma, M)}^{\Delta(a, M)}\left[\lambda^{\sigma} v_{\lambda}\right] \ldots{ }^{I} \mathscr{P}_{(a-L \sigma, M)}^{L \Delta(a, M)}\left[\lambda^{\sigma} v_{\lambda}\right]\right\} \\
& \times \mathscr{P}_{a-(L+1) \sigma,(L+1) \Delta+M}^{a,(L+1) \Delta+M}[\stackrel{0}{\varphi}] .
\end{aligned}
$$

Учитывая то, что

$$
I \mathscr{P}_{(a-j \sigma, M)}^{j \Delta(a, M)}\left[\lambda^{\sigma} v_{\lambda}\right] \leqslant \mathrm{const}{ }^{I} \mathscr{P}_{(a-L \sigma, M)}^{L \Delta(a, M)}\left[\lambda^{\sigma} v_{\lambda}\right]
$$


из (5.22) получаем

$$
\begin{aligned}
& \mathscr{P}_{a, M}^{a, M}\left[\stackrel{0}{\varphi}-B_{k} \omega_{k}\right] \leqslant \frac{\mathrm{const}}{k^{(L+1) \sigma}}\left[{ }^{I} \mathscr{P}_{(a-L \sigma, M)}^{L \Delta(a, M)}\left[\lambda^{\sigma} v_{\lambda}\right]\right]^{L+1} \\
& \times \mathscr{P}_{a-(L+1) \sigma,(L+1) \Delta+M}^{a,(L+1) \Delta+M}[\stackrel{0}{\varphi}] .
\end{aligned}
$$

Без ограничения общности можно считать, что $\sigma<\varepsilon$ (ибо условие (5.4) выполняется с любым $\left.\sigma_{1}<\sigma\right)$. Полагаем $L=\left[\frac{a-\alpha}{\sigma}\right]$. Очевидно, что $(L+1) \sigma>a-\alpha+\varepsilon_{1}$ для некоторого $\varepsilon_{1}>0$ и $a-(L+1) \sigma>\alpha-\varepsilon$. Полагая теперь $Q=(L+1) \Delta+M$, из (5.23) получаем

$$
\mathscr{P}_{a, M}^{a, M}\left[\stackrel{0}{\varphi}-B_{k} \omega_{k}\right] \leqslant \frac{\text { const }}{k^{a-\alpha+\varepsilon_{1}}} \mathscr{P}_{a-\varepsilon, Q}^{a, Q}[\stackrel{0}{\varphi}]
$$

что и доказывает условие (4.32).

Теперь докажем, что выполнено условие (4.31) леммы 5 . Оценим типичное слагаемое суммы (5.19). Для достаточно малых $\varepsilon_{1}$ и $\sigma_{1}$ так же, как и в оценках $(5.22)$, получим

$$
\begin{aligned}
& I_{\alpha-\varepsilon_{1}, 0}^{\mathscr{P}^{a, 0}}\left[B_{0}^{-1}\left(R_{k} B_{0}^{-1}\right)^{j} \stackrel{0}{\varphi}\right] \leqslant \text { const } \mathscr{P}_{\alpha-\varepsilon_{1}, n}^{a, n}\left[\left(R_{k} B_{0}^{-1}\right)^{j}{ }^{0} \varphi\right] \leqslant \cdots \\
& \quad \ldots \leqslant \frac{\mathrm{const}}{k^{j \sigma_{1}}}\left[{ }^{I} \mathscr{P}_{\left(\alpha-\varepsilon_{1}-j \sigma_{1}, M\right)}^{j \Delta(a, M)}\left[\lambda^{\sigma_{1}} v_{\lambda}\right]\right]^{j} \cdot \mathscr{P}_{\alpha-\varepsilon_{1}-j \sigma_{1}, M+(j+1) \Delta}^{a, M+(j+1) \Delta}\left[\begin{array}{l}
0 \\
\varphi
\end{array}\right]
\end{aligned}
$$

Считая $0<\varepsilon_{1}<\varepsilon$ и $0<\sigma_{1}<\frac{\varepsilon-\varepsilon_{1}}{L}$, получим

$$
\alpha-\varepsilon_{1}-j \sigma_{1} \geqslant \alpha-\varepsilon_{1}-L \sigma_{1} \geqslant \alpha-\varepsilon, \quad j=1, \ldots, L .
$$

Отсюда и из (5.24) имеем

$$
{ }_{I} \mathscr{P}_{\alpha-\varepsilon_{1}, 0}^{a, 0}\left[B_{0}^{-1}\left(R_{k} B_{0}^{-1}\right)^{j} \stackrel{0}{\varphi}\right] \leqslant \frac{\mathrm{const}}{k^{j \sigma_{1}}}\left[{ }_{(\alpha-\varepsilon, M)}^{I} \mathscr{P}_{(\alpha, a, M)}^{j \Delta(a)}\left[\lambda^{\sigma_{1}} v_{\lambda}\right]\right]^{j} \cdot \mathscr{P}_{\alpha-\varepsilon, Q}^{a, Q}[\stackrel{0}{\varphi}] .
$$

Мы видим, что каждое слагаемое суммы (5.19), кроме первого, стремится к нулю при $k \rightarrow+\infty$ в пространстве $\mathscr{S}_{\alpha-\varepsilon_{1}, 0}^{a, 0}$, что и доказывает условие (4.31) леммы 5, а вместе с ним и всю теорему. Теорема доказана.

ЗАмЕчАниЕ 1. В условии теоремы вместо (5.4) можно считать, что

$$
\lambda^{\sigma} \eta(\lambda)\left(\stackrel{0}{\varphi}_{\lambda}(t)-\stackrel{0}{\varphi}_{0}(t)\right) \in{ }^{I} F^{q}\left[\mathscr{S}_{\alpha-\varepsilon, M}^{a, M}\right]
$$

где $q \geqslant\left[\frac{a-\alpha}{\sigma}\right](n-M)$, а $n$ берется из леммы 6 об обратном операторе.

ЗАмЕчАнИЕ 2. Если в условиях теоремы отказаться от условия (5.7), то можно только утверждать, что $f(t)$ квазиасимптотически ограничена относительно $\rho(k)$, т.e.

$$
\frac{1}{\rho(k)}|(f(k t), \varphi(t))| \leqslant c_{\varphi}, \quad k>k_{0}, \quad \forall \varphi(t) \in \mathscr{S}_{+} .
$$


ТЕОРема 2. Пусть выполнены все условия теоремы 1, кроме п. 3) (см. формулу (5.4)). Вместо него потребуем, итобы выполнялось следующее условue:

$\left.3^{\prime}\right)$ для любого $M_{1} \geqslant 0$

$$
\stackrel{0}{\varphi}(t)-\stackrel{0}{\varphi}_{\lambda}(t) \underset{\lambda \rightarrow+\infty}{\longrightarrow} 0 \quad \text { o } \quad I \mathscr{S}_{\alpha-\varepsilon, M_{1}}^{a, M_{1}}
$$

Тогда заключение теоремы 1 остается справедливым.

ДокАЗАТЕльство. Так же как и при доказательстве предыдушей теоремы, будем пользоваться леммой 5 . Для этого проведем необходимые предварительные построения. Пусть так же, как и в доказательстве теоремы 1 ,

$$
\eta(\lambda) \in C^{\infty}(0,+\infty), \quad \eta(\lambda)=0 \text { при } \lambda<\lambda_{0}, \quad \eta(\lambda)=1 \text { при } \lambda>\lambda_{1}>\lambda_{0} .
$$

Тогда имеют место включения (5.10) и (5.12), а потому существует такое $n$, что для любого $M_{1} \geqslant 0$ определены линейные непрерывные операторы $B_{0}$ (см.(5.13)) и $B_{0}^{-1}$ (см. (5.14)), причем $B_{0}^{-1}$ является правым обратным к $B_{0}$, и семейство непрерывных операторов $B_{k}$ (см. (5.15)).

Рассмотрим семейство последовательностей

$$
v_{\lambda}(t)=\left\{v_{\lambda}^{\beta}(t), \beta \in I\right\}=\left\{\stackrel{0}{\varphi}_{0}^{\beta}(t)-\stackrel{\varphi}{\varphi}_{\lambda}^{\beta}(t) \eta(\lambda), \beta \in I, \lambda>0\right\} \in{ }^{I} F^{0}\left[\begin{array}{c}
0 \\
\mathscr{S}_{\alpha-\varepsilon}^{a+\varepsilon_{0}, M} \\
\alpha-M
\end{array}\right]
$$

Это семейство порождает последовательность линейных непрерывных операторов

$$
R_{k}:{ }_{I} \mathscr{S}_{0}^{a+\varepsilon, \varepsilon_{0}, 0} \longrightarrow \stackrel{0}{\mathscr{S}}_{\alpha-\varepsilon, M}^{a+\varepsilon_{0}, M}
$$

(см. (3.16), (3.14)). Пусть $\xi(\lambda) \in C^{\infty}(0,+\infty)$, причем $\xi(\lambda)=0$ при $\lambda>1$ и $\xi(\lambda)=1$ при $\lambda<1 / 2$. Для $s>0$ рассмотрим семейства последовательностей функций:

$$
q_{\lambda, s}(t)=\xi\left(\gamma_{s} \lambda\right) v_{\lambda}(t) \quad \text { и } \quad p_{\lambda, s}(t)=\left(1-\xi\left(\gamma_{s} \lambda\right)\right) v_{\lambda}(t)
$$

где функция $\gamma_{s}$ стремится к нулю при $s \rightarrow+\infty$ и будет выбрана позднее. Семейства

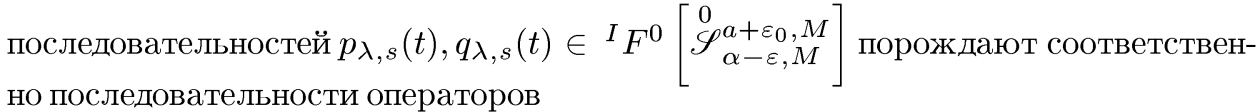

$$
\begin{aligned}
P_{k} \omega & =\sum_{\beta \in I} \int_{0}^{\infty} \omega^{\beta}(\varkappa) p_{k \varkappa, k}^{\beta}\left(\frac{t}{\varkappa}\right) \frac{d \varkappa}{\varkappa}, \\
Q_{k} \omega & =\sum_{\beta \in I} \int_{0}^{\infty} \omega^{\beta}(\varkappa) q_{k \varkappa, k}^{\beta}\left(\frac{t}{\varkappa}\right) \frac{d \varkappa}{\varkappa},
\end{aligned}
$$


линейно и непрерывно отображающих $\underset{I_{0} \mathscr{S}_{\alpha-\varepsilon, 0}^{a+\varepsilon_{0}, 0}}{\stackrel{0}{0} \mathscr{S}_{\alpha-\varepsilon, M}^{a+\varepsilon_{0}, M}}$ (см. следствие $1 \mathrm{k}$ лемме 2). Очевидно, что $P_{k}+Q_{k}=R_{k}$. Согласно лемме П4 для любого $d \in(\alpha-$ $\left.\varepsilon, a+\varepsilon_{0}\right)$ и $\sigma>0$ такого, что $d-\sigma>\alpha-\varepsilon$, имеем

$$
\begin{aligned}
\mathscr{P}_{d, M}^{a, M}\left[Q_{k} \omega\right] & \leqslant \frac{\mathrm{const}}{k^{\sigma}} \cdot{ }^{I} \mathscr{P}_{(d, M)}^{0(a, M)}\left[\lambda^{\sigma} q_{\lambda, k}\right] \cdot{ }_{I} \mathscr{P}_{d-\sigma, 0}^{a, 0}[\omega] \\
& \leqslant \frac{\mathrm{const}}{k^{\sigma}} \frac{1}{\gamma_{k}^{\sigma}} \cdot{ }^{I} \mathscr{P}_{(d, M)}^{0(a, M)}\left[v_{\lambda}\right] \cdot{ }_{I} \mathscr{P}_{d-\sigma, 0}^{a, 0}[\omega]
\end{aligned}
$$

Здесь в последнем неравенстве использована оценка

$$
\begin{aligned}
{ }^{I} \mathscr{P}_{(d, M)}^{0(a, M)}\left[\lambda^{\sigma} q_{\lambda, k}\right] & =\sup _{\beta \in I \lambda>0} \sup _{d, M} \mathscr{P}_{d, M}^{a, M}\left[\lambda^{\sigma} \xi\left(\gamma_{k} \lambda\right) v_{\lambda}^{\beta}(t)\right] \\
& =\sup _{\beta \in I \lambda>0} \sup ^{\sigma} \xi\left(\gamma_{k} \lambda\right) \mathscr{P}_{d, M}^{a, M}\left[v_{\lambda}^{\beta}(t)\right] \leqslant \frac{1}{\gamma_{k}^{\sigma}} \sup _{\beta \in I} \sup _{\lambda>0} \mathscr{P}_{d, M}^{a, M}\left[v_{\lambda}\right] \\
& =\frac{1}{\gamma_{k}^{\sigma}} \cdot{ }^{I} \mathscr{P}_{d, M}^{0(a, M)}\left[v_{\lambda}\right]
\end{aligned}
$$

в которой мы учли, что $\xi\left(\gamma_{k} \lambda\right)=0$ при $\lambda \geqslant 1 / \gamma_{k}$.

Далее, в силу условия (5.26), считая $s$ достаточно большим, так что $\frac{1}{2 \gamma_{s}}>\lambda_{1}$, и замечая, что $p_{\lambda, s}(t)=0$ при $\lambda<1 / 2 \gamma_{s}$ и $\eta(\lambda) \equiv 1$ при $\lambda>\lambda_{1}$, имеем

$$
\left\{p_{\lambda, s}(t)\right\}=\left(1-\xi\left(\gamma_{s} \lambda\right)\right)\left(\stackrel{0}{\varphi}_{0}(t)-\stackrel{0}{\varphi}_{\lambda}(t)\right) \in{ }^{I} F^{0}\left[\begin{array}{c}
0 \\
\mathscr{S}_{\alpha-\varepsilon, M_{1}}^{a}
\end{array}\right] .
$$

Поэтому, еще раз пользуясь (5.26), получим

$$
\begin{aligned}
I \mathscr{P}_{\left(\alpha-\varepsilon, M_{1}\right)}^{0\left(a, M_{1}\right)}\left[p_{\lambda, s}(t)\right] & =\sup _{\beta \in I} \sup _{\lambda \geqslant \frac{1}{2 \gamma_{s}}} \mathscr{P}_{\alpha-\varepsilon, M_{1}}^{a, M_{1}}\left[\left(1-\xi\left(\gamma_{s} \lambda\right)\right)\left(\stackrel{0}{\varphi}_{0}^{\beta}(t)-\stackrel{0}{\varphi}_{\lambda}^{\beta}(t)\right)\right] \\
& \leqslant \sup _{\beta \in I} \sup _{\lambda \geqslant \frac{1}{2 \gamma_{s}}} \mathscr{P}_{\alpha-\varepsilon, M_{1}}^{a, M_{1}}\left[\stackrel{0}{\varphi}_{0}^{\beta}(t)-\stackrel{0}{\varphi}_{\lambda}^{\beta}(t)\right] \leqslant \delta_{s} \underset{s \rightarrow+\infty}{\longrightarrow} 0
\end{aligned}
$$

ибо $1 / 2 \gamma_{s} \rightarrow+\infty$ при $s \rightarrow+\infty$. В силу (5.32) при достаточно больших $k$ для любого $M_{1} \geqslant 0$ имеем семейство линейных непрерьвных отображений

$$
P_{k}: \underset{0}{I_{0}^{a}} \mathscr{S}_{\alpha-\varepsilon, 0}^{a, 0} \longrightarrow \mathscr{S}_{\alpha-\varepsilon, M_{1}}^{a, M_{1}}
$$

Согласно следствию 2 к лемме 2

$$
\begin{aligned}
\mathscr{P}_{\alpha-\varepsilon, M_{1}}^{a, M_{1}}\left[P_{k} \omega\right] & \leqslant \text { const } \cdot{ }^{I} \mathscr{P}_{\left(\alpha-\varepsilon, M_{1}\right)}^{0\left(a, M_{1}\right)}\left[p_{\lambda, k}\right] \cdot{ }_{I} \mathscr{P}_{\alpha-\varepsilon, 0}^{a, 0}[\omega] \\
& \leqslant \mathrm{const} \cdot \delta_{k} \cdot{ }_{I} \mathscr{P}_{\alpha-\varepsilon, 0}^{a, 0}[\omega] .
\end{aligned}
$$

Воспользуемся теперь леммой 5 . Пусть $\varphi(t) \in \mathscr{S}_{\alpha-\varepsilon, M_{1}}^{a, M_{1}}$, где $M_{1}$ достаточно велико $\left(M_{1} \geqslant n, M_{1} \geqslant N\right)$. Подберем $\omega_{k}$ так, чтобы удовлетворялись условия (4.31) и (4.32). Для этого положим

$$
\omega_{k}=\sum_{j=0}^{\infty} B_{0}^{-1}\left(P_{k} B_{0}^{-1}\right)^{j} \stackrel{0}{\varphi}
$$


Из определения (5.14), в котором следует $M_{1}$ положить равньг нулю, а вместо $n$ подставить $M_{1}$, вытекает, что отображение

$$
B_{0}^{-1}: \stackrel{0}{\mathscr{S}}_{\alpha-\varepsilon, M_{1}}^{a, M_{1}} \longrightarrow{ }_{I} \mathscr{S}_{0}^{a, 0} \alpha, 0
$$

линейно и непрерывно. Поэтому в силу (5.34) имеем

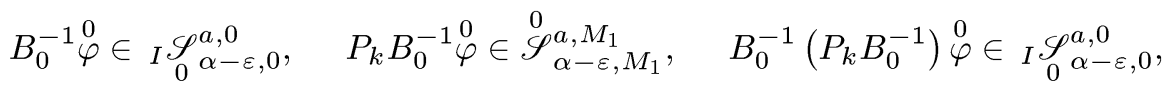

$$
\begin{aligned}
& \left(P_{k} B_{0}^{-1}\right)^{2} \stackrel{0}{\varphi} \in \stackrel{0}{\mathscr{S}^{a}} \alpha, M_{\alpha}, M_{1}, \ldots, \quad\left(P_{k} B_{0}^{-1}\right)^{j} \stackrel{0}{\varphi} \in \stackrel{0}{\mathscr{S}^{a}} \underset{\alpha-\varepsilon, M_{1}}{ }, \\
& B_{0}^{-1}\left(P_{k} B_{0}^{-1}\right)^{j} \stackrel{0}{\varphi} \in{ }_{I}^{S_{0}}{ }_{\alpha-\varepsilon, 0}^{a, 0} .
\end{aligned}
$$

Оценим, пользуясь (5.35) и (5.37), произвольное слагаемое суммы (5.36). Имеем

$$
\begin{aligned}
& { }_{I} \mathscr{P}_{\alpha-\varepsilon, 0}^{a, 0}\left[B_{0}^{-1}\left(P_{k} B_{0}^{-1}\right)^{j}{ }_{\varphi}^{0}\right] \leqslant \operatorname{const} \mathscr{P}_{\alpha-\varepsilon, M_{1}}^{a, M_{1}}\left[\left(P_{k} B_{0}^{-1}\right)^{j}{ }^{0} \varphi\right] \\
& \leqslant \operatorname{const} \delta_{k} \cdot{ }_{I} \mathscr{P}_{\alpha-\varepsilon, 0}^{a, 0}\left[B_{0}^{-1}\left(P_{k} B_{0}^{-1}\right)^{j-1} \stackrel{0}{\varphi}\right] \leqslant \cdots \\
& \quad \cdots \leqslant\left(\operatorname{const} \delta_{k}\right)^{j} \cdot{ }_{I} \mathscr{P}_{\alpha-\varepsilon, 0}^{a, 0}\left[B_{0}^{-1}{ }_{\varphi}^{0}\right] \leqslant \mathrm{const} \cdot\left(\delta_{k} \text { const }\right)^{j} \cdot \mathscr{P}_{\alpha-\varepsilon, M_{1}}^{a, M_{1}}\left[\begin{array}{l}
0 \\
\varphi
\end{array}\right] .
\end{aligned}
$$

Так как $\delta_{k} \rightarrow 0$ при $k \rightarrow \infty$, то при достаточно большом $k$ const $\delta_{k}<1$, что обеспечивает сходимость ряда (5.36) в пространстве ${ }_{I} \mathscr{S}_{\alpha-\varepsilon, 0}^{a, 0}$. Кроме того, очевидно, что $\omega_{k} \rightarrow B_{0}^{-1} \stackrel{0}{\varphi} \equiv \omega$ при $k \rightarrow+\infty$ в $\mathscr{S}_{0}^{a, 0} \alpha, 0$. Итак, условие (4.31) леммы 5 выполнено.

Проверим теперь условие (4.32). Учитывая, что $B_{k}=B_{0}-P_{k}-Q_{k}$, имеем

$$
\begin{aligned}
\stackrel{0}{\varphi}-B_{k} \omega_{k} & =\stackrel{0}{\varphi}-\left(B_{0}-P_{k}-Q_{k}\right) \sum_{j=0}^{\infty} B_{0}^{-1}\left(P_{k} B_{0}^{-1}\right)^{j} \stackrel{0}{\varphi} \\
& =\stackrel{0}{\varphi}-\left(B_{0}-P_{k}\right) B_{0}^{-1} \sum_{j=0}^{\infty}\left(P_{k} B_{0}^{-1}\right)^{j} \stackrel{0}{\varphi}+Q_{k} \omega_{k}=Q_{k} \omega_{k} .
\end{aligned}
$$

Пользуясь (5.31), имеем

$$
\mathscr{P}_{a, M}^{a, M}\left[Q_{k} \omega\right] \leqslant \frac{\text { const }}{k^{\sigma}} \frac{1}{\gamma_{k}^{\sigma}} \cdot{ }^{I} \mathscr{P}_{(a, M)}^{0(a, M)}\left[v_{\lambda}\right] \cdot{ }_{I} \mathscr{P}_{a-\sigma, 0}^{a, 0}[\omega] .
$$

Полагая $\sigma=a-\alpha+\varepsilon_{1}$, где $\varepsilon_{1}<\varepsilon$, получаем

$$
\mathscr{P}_{a, M}^{a, M}\left[\varphi-B_{k} \omega_{k}\right] \leqslant \frac{\mathrm{const}}{k^{a-\alpha+\varepsilon_{1}}} \frac{1}{\gamma_{k}^{a-\alpha+\varepsilon_{1}}} \cdot{ }^{I} \mathscr{P}_{(a, M)}^{0(a, M)}\left[v_{\lambda}\right] \cdot{ }_{I} \mathscr{P}_{\alpha-\varepsilon_{1}, 0}^{a, 0}\left[\omega_{k}\right] .
$$

Учитывая, что ${ }_{I} \mathscr{P}_{a-\varepsilon_{1}, 0}^{a, 0}\left[\omega_{k}\right]<$ const, где const не зависит от $k$, и считая, например, что $\gamma_{s}=1 / \ln s$, убеждаемся в справедливости условия (4.32) леммы 5 . Теорема доказана.

ЗАмЕчАниЕ 1. В формулировке теоремы можно считать, что (5.26) выполнено только для $M_{1}=n$, которое фигурирует в лемме 6 об обратном операторе.

ЗАмЕчАниЕ 2. Если в условиях теоремы 2 отказаться от условия (5.7), то можно только утверждать, что $f(t)$ квазиасимптотически ограничена относительно $\rho(k)$. 


\section{§ 6. Некоторые применения}

В этом параграфе рассматриваются некоторые применения вьшеизложенных результатов. Первое из них относится к квазиасимптотическим свойствам решений обыкновенных дифференциальных уравнений с переменными коэффициентами. Мы изложим его в виде следующей теоремы.

ТЕОрема 3. Пусть $f(t)$ принадлежит $\left(\mathscr{S}_{b}^{a}\right)^{\prime}$ и удовлетворяет уравнению

$$
\sum_{\ell=0}^{n} A_{\ell}(t) \frac{d^{\ell}}{d t^{\ell}} f(t)=g(t), \quad t \geqslant t_{0}>0
$$

где $g(t) \in \mathscr{S}_{+}^{\prime}$, а коэффичиенты $A_{\ell}(t)$ бесконечно дифференцируемы, причем $A_{\ell}(t)=a_{\ell} t^{\ell}\left(1+\gamma_{\ell}(t)\right)$ и существует $\sigma>0$ maкое, что

$$
\left|\left(t \frac{d}{d t}\right)^{j} \gamma_{\ell}(t)\right| \leqslant \frac{c_{j}}{t^{\sigma}}, \quad j=0,1, \ldots, \quad \ell=0,1, \ldots, n,
$$

для некоторых постоянных $c_{j}$. Пусть еще $g(t)$ обладает квазиасимптотикой относительно автомодельной функции $\rho(k)$ порядка $\alpha,-1<\alpha<a, a$ для полинома $P\left(t \frac{d}{d t}\right)=\sum_{\ell=0}^{n} a_{\ell} t^{\ell} \frac{d^{\ell}}{d t^{\ell}}$ выполнено условие

$$
P(-i z) \neq 0, \quad z \in \bar{\Pi}_{\alpha}^{a} .
$$

Тогда $f(t)$ обладает квазиасимптотикой относительно $\rho(k)$.

СлЕДСТВИЕ. Если в условиях теоремы $\alpha<-1$, но

$$
\alpha \neq-1,-2, \ldots
$$

то существуют постоянные $b_{0}, b_{1}, \ldots, b_{\langle\alpha\rangle}$ такие, что $f(t)-\sum_{j=0}^{\langle\alpha\rangle} b_{j} \delta^{(j)}(t)$ обладает квазиасимптотикой относительно $\rho(k)$.

ДОКАЗАТЕЛЬСТВО ТЕОРЕМЫ. В силу конечности порядка $f$ сушествует $Q \geqslant 0$ такое, что $f(t) \in\left(\mathscr{S}_{b, Q}^{a, Q}\right)^{\prime}$. Не нарушая общности, можно считать, что supp $f \subset$ $\left(t_{0},+\infty\right)$. В противном случае положим $f=f_{1}+f_{2}$, где $f_{1}=f$ при $t>t_{0}+1$, а $f_{2}$ имеет компактньй носитель. Так как $\alpha>-1$ и $g_{1}=\sum_{\ell=0}^{n} A_{\ell}(t) \frac{d^{\ell}}{d t^{\ell}} f_{2}(t)$ имеет компактный носитель, то $g_{1}$ обладает тривиальной квазиасимптотикой относительно $\rho(k)$. Нам остается выяснить, обладает ли $f_{1}$ квазиасимптотикой относительно $\rho(k)$.

Итак, пусть $\operatorname{supp} f \subset\left(t_{0},+\infty\right)$; тогда для любого $\varepsilon>0 f(t) \in\left(\mathscr{S}_{\alpha-\varepsilon, Q}^{a, Q}\right)^{\prime}$. Фиксируем $\varepsilon$ настолько малым, чтобы $\alpha-\varepsilon>-1$. Поскольку $g(t)$ обладает квазиасимптотикой относительно $\rho(k)$, то сушествует $N_{0}$ такое, что при $N \geqslant N_{0}$ первообразная порядка $N$ от $g(t)$ является непрерывной функцией и обладает асимптотикой относительно $k^{N} \rho(k)$ (см. [3]), т.е.

$$
\frac{1}{k^{N} \rho(k)}\left(g(t), f_{N}(k-t)\right)=\frac{1}{\rho(k)}\left(g(k t), f_{N}(1-t)\right) \underset{k \rightarrow+\infty}{\longrightarrow} \text { const } .
$$


Пусть теперь $N \geqslant \max \left\{N_{0}, Q+n\right\} ;$ имеем

$$
\begin{aligned}
\frac{1}{\rho(k)} & \left(g(k t), f_{N}(1-t)\right)=\frac{1}{\rho(k)}\left(\sum_{\ell=0}^{n} A_{\ell}(k t) f^{(\ell)}(k t), f_{N}(1-t)\right) \\
& =\frac{1}{\rho(k)}\left(\sum_{\ell=0}^{n} A_{\ell}(k t) \frac{1}{k^{\ell}}\left(\frac{d}{d t}\right)^{\ell} f(k t), f_{N}(1-t)\right) \\
& =\frac{1}{\rho(k)}\left(f(k t), \sum_{\ell=0}^{n} \frac{(-1)^{\ell}}{k^{\ell}}\left(\frac{d}{d t}\right)^{\ell} A_{\ell}(k t) f_{N}(1-t)\right) \\
& =\frac{1}{\rho(k)}\left(f(k t), \sum_{\ell=0}^{n}\left(-\frac{d}{d t}\right)^{\ell} a_{\ell} t^{\ell} f_{N}(1-t)+\sum_{\ell=0}^{n}\left(-\frac{d}{d t}\right)^{\ell} a_{\ell} t^{\ell} \gamma_{\ell}(k t) f_{N}(1-t)\right) \\
& =\frac{1}{\rho(k)}\left(f(k t), \varphi_{k}(t)\right) .
\end{aligned}
$$

В последнем равенстве мы положили

$$
\varphi_{k}(t)=\varphi_{0}(t)+\sum_{\ell=0}^{n}\left(-\frac{d}{d t}\right)^{\ell} a_{\ell} t^{\ell} \gamma_{\ell}(k t) f_{N}(1-t)
$$

где

$$
\varphi_{0}(t)=\sum_{\ell=0}^{n}\left(-\frac{d}{d t}\right)^{\ell} a_{\ell} t^{\ell} f_{N}(1-t)
$$

Воспользуемся теоремой 1 , при этом учтем, что множество $I$ состоит из одного элемента, а в нормах, определяющих пространства $\mathscr{S}_{\alpha-\varepsilon, Q}^{a, Q}$, и преобразовании Меллина отсутствуют тейлоровские многочлены, ибо $\alpha>-1$. Нам нужно показать, что выполнены условия (5.2)-(5.5), которые в нашем случае выглядят так:

$$
\varphi_{k}(t) \underset{k \rightarrow+\infty}{\longrightarrow} \varphi_{0}(t) \text { в } \mathscr{S}_{\alpha-\varepsilon, Q}^{a+\varepsilon_{0}, Q}
$$

найдется $\sigma_{1}$ такое, что

$$
\mathscr{P}_{\alpha-\varepsilon, Q}^{a, Q}\left[\left(k \frac{d}{d k}\right)^{s}\left(\varphi_{k}-\varphi_{0}\right)\right] \leqslant \frac{c_{s}}{k^{\sigma_{1}}}, \quad k>k_{0}, \quad s=0,1, \ldots
$$

найдутся постоянные $A$ и $m$ такие, что

$$
\left|\widehat{\varphi}_{0}(z)\right| \geqslant \frac{A}{1+|z|^{m}} \quad \text { при } \quad z \in \Pi_{\alpha-\varepsilon}^{a+\varepsilon_{0}}
$$

Условие (5.6) в нашем случае отсутствует, а условие (5.7) вытекает из (6.5) и (6.6).

Проверим сначала условие $(6.10)$. Обозначая $\gamma_{\ell}^{*(s)}(k t)=\left(k \frac{d}{d k}\right)^{s} \gamma_{\ell}(k t)$, имеем

$$
\underset{\alpha-\varepsilon, Q}{\mathscr{P}_{0}, Q}\left[\left(k \frac{d}{d k}\right)^{s}\left(\varphi_{k}-\varphi_{0}\right)\right]=\mathscr{P}_{\alpha-\varepsilon, Q}^{a+\varepsilon_{0}, Q}\left[\sum_{\ell=0}^{n}\left(-\frac{d}{d t}\right)^{\ell} a_{\ell} t^{\ell} \gamma_{\ell}^{*(s)}(k t) f_{N}(1-t)\right]
$$


Эта норма оценивается конечной суммой слагаемых вида

$$
\text { const } \int_{0}^{1} t^{\alpha-\varepsilon}\left|\left(t \frac{d}{d t}\right)^{p} f_{N}(1-t) \cdot\left(t \frac{d}{d t}\right)^{q} \gamma_{\ell}^{*(s)}(k t)\right| d t,
$$

где $0 \leqslant p, q \leqslant n+Q, 0 \leqslant \ell \leqslant n$. При этом мы учли, что $\operatorname{supp} f_{N}(1-t) \subset[0,1]$. Далее, учитьвая, что $\left|\left(t \frac{d}{d t}\right)^{p} f_{N}(1-t)\right|<$ const при $p<N$, а также то, что $\left(t \frac{d}{d t}\right)^{q} \gamma_{\ell}^{*(s)}(k t)=\gamma_{\ell}^{*(s+q)}(k t)$, получим, что (6.12) оценивается выражением

$$
\begin{aligned}
\text { const } & \int_{0}^{1} t^{\alpha-\varepsilon}\left|\gamma_{\ell}^{*(q+s)}(k t)\right| d t=\frac{\text { const }}{k^{\alpha-\varepsilon+1}} \int_{0}^{k} t^{\alpha-\varepsilon}\left|\gamma_{\ell}^{*(q+s)}(t)\right| d t \\
& \leqslant \frac{\text { const }}{k^{\alpha-\varepsilon+1}}\left[\int_{0}^{1} t^{\alpha-\varepsilon}\left|\gamma_{\ell}^{*(q+s)}(t)\right| d t+\int_{1}^{k} t^{\alpha-\varepsilon}\left|\gamma_{\ell}^{*(q+s)}(t)\right| d t\right] \\
& \leqslant \frac{\text { const }}{k^{\alpha-\varepsilon+1}}+\frac{\text { const }}{k^{\alpha-\varepsilon+1}} \int_{1}^{k} t^{\alpha-\varepsilon-\sigma} d t \leqslant \frac{\text { const }}{k^{\alpha-\varepsilon+1}}+\frac{\text { const }}{k^{\sigma}} .
\end{aligned}
$$

Полагая $\sigma_{1}=\min \{\sigma, \alpha-\varepsilon+1\}$, получим (6.10).

Условие (6.9) следует из (6.10) при $s=0$. Проверим условие (6.11).

$$
\begin{aligned}
\mathscr{M}\left[\varphi_{0}(t)\right] & =\mathscr{M}\left[\sum_{\ell=0}^{n}(-1)^{\ell} a_{\lambda}\left(\frac{d}{d t}\right)^{\ell} t^{\ell} f_{N}(1-t)\right] \\
& =\int_{0}^{\infty} t^{-i z} \sum_{\ell=0}^{n} a_{\ell}\left(-\frac{d}{d t}\right)^{\ell} t^{\ell} f_{N}(1-t) d t \\
& =\int_{0}^{\infty}\left(\sum_{\ell=0}^{n} a_{\ell} t^{\ell}\left(\frac{d}{d t}\right)^{\ell} t^{-i z}\right) f_{N}(1-t) d t \\
& =\int_{0}^{\infty}\left(P\left(t \frac{d}{d t}\right) t^{-i z}\right) f_{N}(1-t) d t=P(-i z) \int_{0}^{\infty} t^{-i z} f_{N}(1-t) d t \\
& =P(-i z) \frac{\Gamma(1-i z)}{\Gamma(N+1-i z)} .
\end{aligned}
$$

Учитывая (6.3) и тот факт, что

$$
\frac{\Gamma(1-i z)}{\Gamma(N+1-i z)}=z^{-N}(1+o(1)), \quad|z| \rightarrow \infty, \quad \text { при } z \in \Pi_{\alpha-\varepsilon}^{a+\varepsilon_{0}}
$$

и что функция $\Gamma(N+1-i z)$ не имеет полюсов в этой полосе, получим (6.11). Теорема доказана.

ДоКАЗАТЕЛЬСтво СЛЕДСТВИЯ. Пусть $\alpha<-1$. Умножим уравнение (6.1) на $t$. Перегруппировывая производные и перестраивая коэффициенты, имеем

$$
\operatorname{tg}(t)=\sum_{\ell=0}^{n} t A_{\ell}(t)\left(\frac{d}{d t}\right)^{\ell} f(t)=\sum_{\ell=0}^{n} A_{\ell}^{1}(t)\left(\frac{d}{d t}\right)^{\ell}(t f(t))
$$


где $A_{n}^{1}(t)=A_{n}(t), A_{\ell}^{1}(t)=A_{\ell}(t)-\frac{\ell+1}{t} A_{\ell+1}^{1}(t), \ell=n-1, n-2, \ldots, 1,0$. Откуда видно, что новые коэффициенты $A_{\ell}^{1}$ удовлетворяют условию (6.2). Проделывая эту процедуру $N$ раз, $N \geqslant-\alpha$, получим уравнение

$$
\sum_{\ell=0}^{n} A_{\ell}^{N}(t)\left(\frac{d}{d t}\right)^{\ell}\left(t^{N} f(t)\right)=t^{N} g(t)
$$

в котором коэффициенты удовлетворяют условию (6.2), а правая часть обладает квазиасимптотикой относительно автомодельной функщии $k^{N} \rho(k)$ порядка $\alpha+N>0$.

При такой перестройке главная часть уравнения также перестроится следующим образом:

$$
t^{N} \sum_{\ell=0}^{n} a_{\ell} t^{\ell}\left(\frac{d}{d t}\right)^{\ell} f=t^{N} P\left(t \frac{d}{d t}\right) f=t^{N} P\left(t \frac{d}{d t}\right)\left(\frac{1}{t^{N}} t^{N} f\right)=P_{N}\left(t \frac{d}{d t}\right)\left(t^{N} f\right) .
$$

Нетрудно проверить, что новая главная часть $P_{N}\left(t \frac{d}{d t}\right)=t^{N} P\left(t \frac{d}{d t}\right) \frac{1}{t^{N}}$ удовлетворяет условию

$$
P_{N}(-i z)=P(-i(z-i N)) \neq 0 \text { при } z \in \bar{\Pi}_{\alpha+N}^{a+N} .
$$

Так как $\alpha+N>0$, то по теореме $3 t^{N} f(t)$ обладает квазиасимптотикой относительно $k^{N} \rho(k)$. По теореме о делении (см. [3, лемма 2]) существуют постоянные $b_{0}, b_{1}, \ldots, b_{p}$ такие, что

$$
\frac{1}{\rho(k)}\left(f(k t)-\sum_{j=0}^{p} b_{j} \delta^{(j)}(k t), \varphi(t)\right) \underset{k \rightarrow+\infty}{\longrightarrow} c_{\varphi} \quad \forall \varphi \in \mathscr{I}_{+} .
$$

Учитьвая, что

$$
\frac{1}{\rho(k)}\left(\sum_{j=\langle\alpha+1\rangle}^{p} b_{j} \delta^{(j)}(k t), \varphi(t)\right) \underset{k \rightarrow+\infty}{\longrightarrow} 0,
$$

из (6.15) завершаем доказательство.

Второе применение относится к вопросам о некомпенсации особенностей голоморфных функций.

ТЕОРема 4. Пусть $\tilde{f}(z) \in H\left(\mathbb{R}_{+}\right)$, функция $\rho(k)$ автомодельна порядка $\alpha$, а $\gamma(z)$ и $g(z)$ - комплекснозначные непрерывные при $\operatorname{Im} z>0$ функиии такие, чmo

$$
\begin{gathered}
\gamma(z) \longrightarrow 0 \quad n p u \quad|z| \rightarrow 0, \quad \operatorname{Im} z>0, \\
\{z: z=g(\zeta), \zeta \in \mathbb{C}, \operatorname{Im} z>0\} \subset \mathscr{K} \Subset T^{\mathbb{R}_{+}},
\end{gathered}
$$

где $\mathscr{K}$ - некоторый компакт в верхней полуплоскости.

Если существуют постоянные $c, r_{0}$ и $m$ такие, что

$$
|\tilde{f}(z)+\gamma(z) \tilde{f}(|z| g(z))| \leqslant \frac{c \rho(1 /|z|)}{(\sin \arg z)^{m}}, \quad|z|<r_{0}
$$

то существуют постоянные $c_{1}$ и $r_{1}$ такие, что

$$
|\tilde{f}(z)| \leqslant \frac{c_{1} \rho(1 /|z|)}{(\sin \arg z)^{m}}, \quad|z|<r_{1}
$$


ДокАЗАТЕЛЬСТво. Так как $\tilde{f}(z) \in H\left(\mathbb{R}_{+}\right)$, то существует обобщенная функция $f(t) \in\left(\mathscr{S}_{b, N, \delta}^{a, M}\right)^{\prime}$ такая, что $L[f]=\tilde{f}$ (см. (1.7)). Не нарушая обшности, считаем, что $a>-1$ и $a>\alpha$. Полагая $|z|=1 / k$ и $\arg z=\beta, \beta \in I=\{(0, \pi)\}$, имеем

$$
\begin{aligned}
\tilde{f}(z)+\gamma(z) \tilde{f}(|z| g(z)) & =\left(f(t), e^{i z t}+\gamma(z) e^{i g(z)|z| t}\right) \\
& =\left(f(k t), e^{i t e^{i \beta}}+\gamma\left(\frac{1}{k} e^{i \beta}\right) e^{i g\left((1 / k) e^{i \beta}\right) t}\right)
\end{aligned}
$$

Здесь мы сделали замену переменных $t^{\prime}=|z| t$ и, как обычно, опустили штрихи. Обозначая

$$
\varphi_{k}^{\beta}(t)=(\sin \beta)^{Q}\left(e^{i e^{i \beta} t}+\gamma\left(\frac{1}{k} e^{i \beta}\right) e^{i g\left((1 / k) e^{i \beta}\right) t}\right)
$$

где $Q$ достаточно велико, например $Q \geqslant[a]+2$, имеем

$$
\frac{\sin ^{Q} \arg z}{\rho(1 /|z|)}(\tilde{f}(z)+\gamma(z) \tilde{f}(|z| g(z)))=\frac{1}{\rho(k)}\left(f(k t), \varphi_{k}^{\beta}(t)\right)
$$

Воспользуемся теоремой 2. Проверим, что все условия этой теоремы для семейства последовательностей (6.20) выполнены. Как и в примере 1 , нетрудно убедиться в том, что $\sup _{\beta \in I} \sup _{k>k_{0}} \mathscr{P}_{b, N, \delta}^{a+\varepsilon_{0}, M}\left[\varphi_{k}^{\beta}(t)\right]<\infty$, где $\varepsilon_{0}$ достаточно мало, так что

$$
\left\{\varphi_{k}^{\beta}(t), \beta \in I, k>k_{0}\right\} \equiv \varphi_{k}(t) \in{ }^{I} F^{0}\left[\mathscr{P}_{b, N, \delta}^{a+\varepsilon_{0}, M}\right]
$$

причем

$$
\forall \beta \in I \quad \varphi_{k}^{\beta}(t) \underset{k \rightarrow+\infty}{\longrightarrow} \varphi_{0}^{\beta}(t)=(\sin \beta)^{Q} e^{i e^{i \beta} t} \quad \text { в } \quad \mathscr{S}_{b, N, \delta}^{a+\varepsilon_{0}, M} .
$$

Проверим условие (5.26). Для любого $M_{1}$ в силу (6.16) имеем

$$
\begin{aligned}
\sup _{\beta \in I} \mathscr{P}_{\alpha-\varepsilon, M_{1}}^{a, M_{1}}\left[\varphi_{k}^{\beta}(t)-\varphi_{0}^{\beta}(t)\right] \\
\quad=\sup _{\beta \in I} \mathscr{P}_{\alpha-\varepsilon, M_{1}}^{a, M_{1}}\left[(\sin \beta)^{Q} \gamma\left(\frac{1}{k} e^{i \beta}\right) e^{i g\left((1 / k) e^{i \beta}\right) t}\right] \underset{k \rightarrow \infty}{\longrightarrow} 0 .
\end{aligned}
$$

Здесь учтено, что $a>-1$, а потому $\stackrel{0}{\varphi}(t)=\varphi(t)$. Покажем, что выполнено и условие (5.5). Имеем

$$
\widehat{\varphi}_{0}^{\beta}(z)=\mathscr{M}\left[(\sin \beta)^{Q} e^{i e^{i \beta} t}\right]=(\sin \beta)^{Q}\left(-i e^{i \beta}\right)^{(i z-1)} \Gamma(1-i z)
$$

(см., например, [16]), так что для $z=x+i y$

$$
\left|\widehat{\varphi}_{0}^{\beta}(z)\right|=(\sin \beta)^{Q}\left|e^{-(\beta-\pi / 2) z} \Gamma(1+y-i x)\right| .
$$


В полосе $z \in \Pi_{\alpha-\varepsilon}^{a+\varepsilon_{0}}$ при $x<-N$, где $N$ достаточно велико, справедлива оценка

$$
\begin{aligned}
\sup _{\beta \in I}\left|\widehat{\varphi}_{0}^{\beta}(z)\right| & \geqslant\left|\widehat{\varphi}_{0}^{\pi+1 / x}(x+i y)\right| \geqslant \operatorname{const} e^{-(\pi / 2) x} e^{-(\pi / 2)|x|}|x|^{y+1 / 2}\left|\sin \left(\pi+\frac{1}{x}\right)\right|^{Q} \\
& \geqslant \text { const }|x|^{b+1 / 2-Q} .
\end{aligned}
$$

Здесь учтено, что $\sin (\pi+1 / x) \sim 1 /|x|$ при больших $|x|$, а также то, что

$$
\begin{gathered}
\Gamma(1+y-i x)=\sqrt{2 \pi} e^{-(\pi / 2)|x|}|x|^{1 / 2+y}(1+o(1)), \\
\Gamma(s-1-y+i x)=\sqrt{2 \pi} e^{-(\pi / 2)|x|}|x|^{-3 / 2+s+y}(1+o(1)) .
\end{gathered}
$$

Аналогичную оценку при $x \rightarrow+\infty$ получим, выбирая $\beta=1 / x$. Теперь нетрудно усмотреть, что условие (5.5) выполнено. Согласно теореме 2 (см. замечание 2 ) обобщенная функция $f(t)$ квазиасимптотически ограничена относительно $\rho(k)$. По общей тауберовой теореме [3] существуют постоянные $B, c_{2}$ и $r_{1}$ такие, что $|\tilde{f}(z)| \leqslant$ $\frac{c_{2} \rho(1 /|z|)}{(\sin \arg z)^{B}}$ при $|z|<r_{1}$. Откуда с учетом (6.16)-(6.18) имеем

$$
\frac{1}{\rho(1 /|z|)}|\gamma(z) \tilde{f}(|z| g(z))| \longrightarrow 0 \text { при }|z| \rightarrow 0 .
$$

Из (6.18) и (6.22) следует (6.19). Теорема доказана.

\section{§ 7. Абелевы и тауберовы теоремы для интегральных преобразований типа Стилтьеса}

Как другое применение полученных выше результатов мы докажем абелевы и тауберовы теоремы для интегральных преобразований обобшенных функций таких, как обобщенное интегральное преобразование Стилтьеса, интегральные преобразования с логарифмическим ядром и с ядром арктангенса. Для этого нам понадобится некоторый вариант теоремы 1 , рассмотренной в $\S 5$, который представляет и самостоятельный интерес (отметим, что результаты этого параграфа в несколько другой формулировке анонсированы без доказательства в [21]). В этом варианте мы будем рассматривать семейства последовательностей, фактически не зависящие от $\lambda$, что, конечно, упрошает ситуацию. Однако при этом мы хотим отказаться от условий а) и б) (см. (5.5) и (5.6)). В частности, некоторые из производных $\frac{d^{j}}{d t^{j}} \varphi_{0}^{\beta}(0)$ могут обрашаться в нуль при всех $\beta \in J$. Что касается условия а), то будем предполагать, что оно вьполняется вне некоторого компакта, а на этом компакте $\widehat{\varphi}_{0}^{\beta}(z)$ могут иметь общие нули. В этой ситуации заключение теоремы 1 для обобщенной функции $f$ перестает быть верным. Тем не менее, оно останется справедливым, если предварительно из $f$ вычесть конечное число контрчленов определенного вида. Множество этих контрчленов образует конечномерное линейное пространство $\mathscr{K}_{\alpha}^{a}\left[\varphi_{0}(t)\right]$, которое мы далее опишем.

ОПРЕДЕЛЕНИЕ 8. Пусть заданы числа: не целые $a, b, b<a$, целые неотрицательные $M, N$, а также $\delta>0$ и $\alpha, b<\alpha<a$. Назовем семейство функций

$$
\varphi_{0}(t)=\left\{\varphi_{0}^{\beta}(t), \beta \in J\right\}
$$


допустимылм, если сушествует $\varepsilon>0$ такое, что $\varphi_{0}^{\beta}(t) \in \mathscr{S}_{b, N, \delta}^{a+\varepsilon, M}$ для любого $\beta \in J$, и если выполнены условия:

$$
\sup _{\beta \in J} \mathscr{P}_{b, N, \delta}^{a+\varepsilon, M}\left[\varphi_{0}^{\beta}(t)\right]<\infty
$$

существуют постоянные $A, m$ и $R_{0}$ такие, что

$$
\sup _{\beta \in J}\left|\widehat{\varphi}_{0}^{\beta}(z)\right| \geqslant \frac{A}{1+|z|^{m}}, \quad z \in \Pi_{\alpha-\varepsilon}^{a+\varepsilon}, \quad|z|>R_{0} .
$$

Кроме того, преобразование Меллина этого семейства $\widehat{\varphi}_{0}(z)$ не имеет общих нулей при $\operatorname{Im} z=\alpha$, а если $\alpha=-1,-2, \ldots$, то в точке $z=i \alpha$ хотя бы одна из функций семейства имеет полюс с вычетом, отличным от нуля.

ЗАМЕЧАНИЕ 1 . Пусть заданы числа $a, b, M, N, \delta$ и $b<\alpha<a$, а также допустимое семейство (7.1). Опишем базис соответствующего этому семейству пространства контрчленов $\mathscr{K}_{\alpha}^{a}\left[\varphi_{0}(t)\right]$. Рассмотрим следуюшие три случая:

1) пусть для некоторого $j, 0 \leqslant j \leqslant\langle a\rangle$, имеем $\frac{d^{j}}{d t^{j}} \varphi_{0}^{\beta}(0)=0$ для всех $\beta \in J$; тогда $\mathscr{K}_{\alpha}^{a}\left[\varphi_{0}(t)\right]$ содержит $\delta^{(j)}(t)$ (если $a>-1$, то этот случай отсутствует);

2) пусть в точке $-i n, n=1,2, \ldots, \alpha<-n<a$, семейство $\left\{(z+i n) \widehat{\varphi}_{0}^{\beta}(z)\right.$, $\beta \in J\}$ имеет нуль кратности $h$; тогда $\mathscr{K}_{\alpha}^{a}\left[\varphi_{0}(t)\right]$ содержит обобщенные функции

$$
\delta^{(n-1)}(t), \quad t_{+}^{-n}, \quad t_{+}^{-n} \ln t_{+}, \quad \ldots, t_{+}^{-n} \ln ^{h-2} t_{+}
$$

$\left(\right.$ если $h=1$, то $\mathscr{K}_{\alpha}^{a}\left[\varphi_{0}(t)\right]$ содержит только $\left.\delta^{(n-1)}(t)\right)$;

$3)$ пусть в точке $\zeta, \zeta \neq-i,-2 i, \ldots, \alpha<\operatorname{Im} \zeta \leqslant a$, семейство $\widehat{\varphi}_{0}(z)$ имеет нуль кратности $r$; тогда $\mathscr{K}_{\alpha}^{a}\left[\varphi_{0}(t)\right]$ содержит обобщенные функции

$$
t_{+}^{-i \zeta}, \quad t_{+}^{-i \zeta} \ln t_{+}, \quad \ldots, t_{+}^{-i \zeta} \ln ^{r-1} t_{+}
$$

ЗАМЕЧАНИЕ 2. Пусть

$$
j_{1}, \ldots, j_{p}, \quad 0 \leqslant j_{s} \leqslant\langle a\rangle \text { для } s=1, \ldots, p,
$$

- те самые $j$, которые фигурируют в 1$),$ и пусть семейство $\widehat{\varphi}_{0}(z)$ имеет нули в точKax

$$
\zeta_{q} \in \bar{\Pi}_{\alpha}^{a}, \quad q=1, \ldots, \ell, \quad \zeta_{q} \neq-i,-2 i, \ldots,
$$

кратности $r_{q}$ соответственно. Кроме того, пусть в точках

$$
z_{\tau} \in \bar{\Pi}_{\alpha}^{a}, \quad z_{\tau}=-i n_{\tau}, \quad n_{\tau} \in \mathbb{N}_{+}, \quad \tau=1,2, \ldots, w
$$

семейства $\left(z-z_{\tau}\right) \widehat{\varphi}_{0}(z)=\left\{\left(z-z_{\tau}\right) \widehat{\varphi}_{0}^{\beta}(z), \beta \in J\right\}$, имеют нули кратности $h_{\tau}$ соответственно. Тогда любое $f_{0} \in \mathscr{K}_{\alpha}^{a}\left[\varphi_{0}(t)\right]$ представляется в виде

$$
\begin{aligned}
f_{0}= & \sum_{q=1}^{\ell} \sum_{\gamma=0}^{r_{q}-1} c_{q \gamma} t_{+}^{-i \zeta_{q}} \ln ^{\gamma} t_{+}+\sum_{\tau=1}^{w} \sum_{\xi=1}^{h_{\tau}-1} a_{\xi \tau} t_{+}^{-n_{\tau}} \ln ^{\xi-1} t_{+} \\
& +\sum_{\tau=1}^{w} d_{\tau} \delta^{\left(n_{\tau}-1\right)}(t)+\sum_{s=1}^{p} b_{s} \delta^{\left(j_{s}\right)}(t),
\end{aligned}
$$

где $d_{\tau}, b_{s}, c_{q \gamma}, a_{\xi \tau}-$ некоторые постоянные. 
TEOPEмA 5. Пусть $f \in\left(\mathscr{S}_{b, N, \delta}^{a, M}\right)^{\prime}, a>b, \quad N \geqslant M, \rho(k)$ - автомодельная функиия порядка $\alpha, \quad b<\alpha<a$, и семейство функиий

$$
\varphi_{0}(t)=\left\{\varphi_{0}^{\beta}(t), \beta \in J\right\}
$$

допустимо относительно чисел $a, b, M, N, \delta, \alpha$ (см. определение 8).

Если $f$ обладает ограниченной квазиасимптотикой по $\beta \in J$ относительно $\rho(k)$ на семействе $\varphi_{0}(t)$, т.е. для любого $\beta \in J$

$$
\frac{1}{\rho(k)}\left(f(k t), \varphi_{0}^{\beta}(t)\right) \underset{k \rightarrow+\infty}{\longrightarrow} c^{\beta}, \quad \frac{1}{\rho(k)}\left|\left(f(k t), \varphi_{0}^{\beta}(t)\right)\right|<C, \quad k>k_{0},
$$

для некоторой постоянной $C$, то найдется обобщенная функщия $f_{0} \in \mathscr{K}_{\alpha}^{a}\left[\varphi_{0}(t)\right]$ такая, что $F=f-f_{0}$ обладает квазиасимптотикой относительно $\rho(k)$, т.е.

$$
\frac{1}{\rho(k)}(F(k t), \varphi(t)) \underset{k \rightarrow+\infty}{\longrightarrow} c_{\varphi} \quad \forall \varphi(t) \in \mathscr{S}_{+} .
$$

ДокАЗАТЕЛЬСтво. Сначала покажем, что $f$ обладает квазиасимптотикой относительно $\rho(k)$ на основных функциях $\varphi(t) \in \mathscr{S}_{+}$, имеющих те же нули производных в нуле и те же нули и полюсы преобразования Меллина, что и у семейства $\varphi_{0}(t)$. Другими словами, рассматривая $\varphi(t) \in \mathscr{S}_{+}$как семейство, состоящее из одной функции, имеем включение $\mathscr{K}_{\alpha}^{a}[\varphi(t)] \supset \mathscr{K}_{\alpha}^{a}\left[\varphi_{0}(t)\right]$. Воспользуемся леммой 5 с учетом замечания к ней. Для этого введем полином

$$
W(z)=\prod_{s=1}^{p}\left(z+i\left(j_{s}+1\right)\right) \prod_{q=1}^{\ell}\left(z-\zeta_{q}\right)^{r_{q}} \prod_{\tau=1}^{w}\left(z+i n_{\tau}\right)^{h_{\tau}}
$$

где $j_{s}, p, \zeta_{q}, \ell, r_{q}, n_{\tau}, w$ и $h_{\tau}$ - те самые, что фигурируют при описании $\mathscr{K}_{\alpha}^{a}\left[\varphi_{0}(t)\right]$ для семейства (7.8) аналогично формулам (7.4)-(7.6), и семейство функций $\left\{\widehat{\varphi}_{1}^{\beta}(z)=\frac{1}{W(z)} \widehat{\varphi}_{0}^{\beta}, \beta \in J\right\}=\widehat{\varphi}_{1}(z)$. Из леммы П0, замечания к ней и леммы П1 вытекает, что существуют счетное множество $I \subset J, \lambda_{\beta}>0, \beta \in I$, и семейство $g_{\beta}(z)$ аналитических в полосе $\Pi_{\alpha-\varepsilon}^{a+\varepsilon}$ функций (полиномиально ограниченных там полиномом, не зависяшим от $\beta$ ) такие, что

$$
\sum_{\beta \in I} \lambda_{\beta}<\infty, \quad \sum_{\beta \in I} \lambda_{\beta} g_{\beta}(z) \hat{\varphi}_{1}^{\beta}(z)=1 \text { при } z \in \Pi_{\alpha-\varepsilon}^{a+\varepsilon} .
$$

Рассмотрим оператор $B_{0}$, определяемый формулой (3.3) по семейству (7.8). Покажем, что сушествует семейство функций $\omega \in{ }_{I}^{\mathscr{S}_{0}^{a} \alpha-\varepsilon, 0}$, , где $0<\varepsilon<\varepsilon_{0}$, такое, что $B_{0} \omega=\stackrel{0}{\varphi}$. Для этого положим

$$
\widehat{\omega}^{\beta}(z)=\lambda_{\beta} g_{\beta}(z) \frac{\widehat{\varphi}(z)}{W(z)}, \quad \text { тогда } \widehat{\varphi}(z)=\sum_{\beta \in I} \widehat{\omega}^{\beta}(z) \widehat{\varphi}_{0}^{\beta}(z) .
$$


Отметим, что $\widehat{\omega}^{\beta}(z)$ аналитичны в $\Pi_{\alpha-\varepsilon}^{a+\varepsilon}$ и убывают на бесконечности быстрее любой степени $|z|^{-1}$, а потому, переходя к обратному преобразованию Меллина в (7.11) и уменьшая, если необходимо, $\varepsilon$, получим

$$
\stackrel{0}{\varphi}(t)=B_{0} \omega, \quad\left\{\omega^{\beta}(t) \in \mathscr{S}_{0}^{a, \infty} \alpha, \infty, \beta \in I\right\}=\omega(t) \in{\underset{I}{I}}_{0}^{\mathscr{S}_{\alpha-\varepsilon, \infty}^{a, \infty}} .
$$

Теперь положим $\omega_{k}=\omega$; видим, что условие (4.31) леммы 5 выполняется автоматически. Учитьвая, что

$$
B_{k} \omega_{k}=B_{k} \omega=\sum_{\beta \in I} \int_{0}^{\infty} \omega^{\beta}(\varkappa)^{0} \varphi_{0}^{\beta}\left(\frac{t}{\varkappa}\right) \frac{d \varkappa}{\varkappa}=B_{0} \omega,
$$

видим, что условие (4.32) также тривиально вьполнено. Таким образом, $f$ обладает квазиасимптотикой относительно $\rho(k)$ на таких $\varphi$.

Пусть $\psi(t)$ - произвольная функция из $\mathscr{S}_{+}$. Тогда функция $\varphi(t)=W\left(-i \frac{d}{d t} t\right) \psi(t)$ является функцией уже рассмотренного вида. По уже доказанному

$$
\begin{aligned}
& \frac{1}{\rho(k)}(f(k t), \varphi(t))=\frac{1}{\rho(k)}\left(f(k t), W\left(-i \frac{d}{d t} t\right) \psi(t)\right) \\
& \quad=\frac{1}{\rho(k)}\left(W\left(i t \frac{d}{d t}\right) f(k t), \psi(t)\right)=\frac{1}{\rho(k)}(g(t), \psi(t)) \underset{k \rightarrow+\infty}{\longrightarrow} c_{\psi},
\end{aligned}
$$

где

$$
W\left(i t \frac{d}{d t}\right) f=g
$$

при этом обобщенная функция $g$ (согласно (7.12)) обладает квазиасимптотикой относительно автомодельной функции $\rho(k)$ порядка $\alpha$. Так как мнимая часть всех корней полинома $W(z)$ отлична от $\alpha$ (см. определение 8 ), то по лемме П6 найдется решение $F$ уравнения (7.13), обладающее квазиасимптотикой относительно $\rho(k)$. Но наше решение $f$ отличается от $F$ на некоторое решение $f_{0}$ однородного уравнения

$$
W\left(i t \frac{d}{d t}\right) f_{0}=0, \quad f_{0} \in \mathscr{S}_{+}^{\prime},
$$

любое решение которого согласно (1.10)-(1.13) принадлежит $\mathscr{K}_{\alpha}^{a}\left[\varphi_{0}\right]$. Теорема доказана.

Обобщенное интегральное преобразование Стилтьеса задается формулой

$$
F(z)=\left(f(t), \frac{\ln ^{n}\left(c-\frac{t}{z}\right)}{\left(c-\frac{t}{z}\right)^{s}}\right), \quad z \in \mathbb{C} \backslash[0,+\infty) .
$$

Здесь $n=0,1, \ldots,-\infty<s<+\infty, c>0$, а логарифм задается своей главной ветвью: $\ln z=\ln |z|+i \arg z,|\arg z|<\pi$. Ядра этих интегральных преобразований

$$
\psi\left(\frac{t}{z}\right)=\frac{\ln ^{n}\left(c-\frac{t}{z}\right)}{\left(c-\frac{t}{z}\right)^{s}} \in \mathscr{S}_{b}^{a}, \quad z \in \mathbb{C} \backslash[0,+\infty),
$$

для любого $a<s-1$ и любого $b<a$. Формула (7.14) для $f \in\left(\mathscr{S}_{b}^{a}\right)^{\prime}$ определяет аналитическую функцию $F(z)$ во всей комплексной плоскости $\mathbb{C}$ с разрезом по положительной части вещественной оси.

Справедлива следуюшая абелева 
Tеорема 6. Пусть $f(t) \in\left(\mathscr{S}_{b}^{a}\right)^{\prime}, \quad a<s-1, \quad b<a$, обладает квазиасимлтотикой относительно автомодельной функции $\rho(k)$ порядка $\alpha, b<\alpha<a$, m.e.

$$
\frac{1}{\rho(k)}(f(k t), \varphi(t)) \underset{k \rightarrow+\infty}{\longrightarrow} c_{\varphi} \quad \forall \varphi \in \mathscr{S}_{+} .
$$

Тогда имеет место:

1) существует предел

$$
\frac{1}{r \rho(r)} F\left(r e^{i \beta}\right) \underset{r \rightarrow+\infty}{\longrightarrow} c^{\beta} \quad \forall \beta \in(0,2 \pi)
$$

2) существуют $A, m$ и $r_{0}$ такие, что

$$
\frac{1}{r \rho(r)}\left|F\left(r e^{i \beta}\right)\right| \leqslant \frac{A}{\sin ^{m} \frac{\beta}{2}}, \quad r>r_{0}, \quad \beta \in(0,2 \pi) .
$$

ДоКАЗАТЕЛЬСТВо. В силу (7.16) и теоремы о равномерной ограниченности (см. [22]) последовательность обобщенных функций $\frac{1}{\rho(k)} f(k t)$ сходится на всех функциях $\varphi(t) \in \mathscr{S}_{b, Q}^{a, Q}$ при некотором $Q \geqslant 0$. Учитывая, что

$$
\psi^{\beta}(t)=\frac{\ln ^{n}\left(c-t e^{-i \beta}\right)}{\left(c-t e^{-i \beta}\right)^{s}} \in \mathscr{S}_{b, Q}^{a, Q} \text { при любом } Q \geqslant 0 \text { и } 0<\beta<2 \pi,
$$

полагая $z=r e^{i \beta}$ и делая замену переменных $t=r t^{\prime}$, опуская, как обьчно, штрихи, имеем

$$
\begin{aligned}
\frac{1}{r \rho(r)} F\left(r e^{i \beta}\right) & =\frac{1}{r \rho(r)}\left(f(t), \frac{\ln ^{n}\left(c-\frac{t}{r} e^{-i \beta}\right)}{\left(c-\frac{t}{r} e^{-i \beta}\right)^{s}}\right) \\
& =\frac{1}{\rho(r)}\left(f(r t), \frac{\ln ^{n}\left(c-t e^{-i \beta}\right)}{\left(c-t e^{-i \beta}\right)^{s}}\right) \underset{r \rightarrow+\infty}{\longrightarrow} c^{\beta}
\end{aligned}
$$

что и доказывает (7.17). Из легко проверяемой оценки

$$
\mathscr{P}_{b, Q}^{a, Q}\left[\psi^{\beta}(t)\right] \leqslant \frac{\text { const }}{\sin ^{Q+s+1} \frac{\beta}{2}}
$$

и сходимости последовательности функционалов $\frac{1}{\rho(k)} f(k t)$ вытекает, что

$$
\frac{1}{r \rho(r)}\left|F\left(r e^{i \beta}\right)\right|=\frac{1}{\rho(r)}\left|\left(f(r t), \psi^{\beta}(t)\right)\right| \leqslant \text { const } \mathscr{P}_{b, Q}^{a, Q}\left[\psi^{\beta}(t)\right] \leqslant \frac{\text { const }}{\sin ^{Q+s+1} \frac{\beta}{2}} .
$$

Откуда и следует (7.18) при $m \geqslant Q+s+1$. Теорема доказана.

Обратную (тауберову) теорему мы докажем для случая $n=1$. 
Теорема 7. Пусть $F(z)$ - аналитическая в $\mathbb{C} \backslash[0,+\infty)$ функиия, определяемая формулой

$$
F(z)=\left(f(t), \frac{\ln \left(c-\frac{t}{z}\right)}{\left(c-\frac{t}{z}\right)^{s}}\right), \quad z \in \mathbb{C} \backslash[0,+\infty),
$$

где $f(t) \in\left(\mathscr{S}_{b}^{a}\right)^{\prime}, \quad b<a<s-1, \quad s>0, a \rho(k)$ - автомодельная функция порядка $\alpha, b<\alpha<a$, и выполнены условия (7.17) и (7.18). Обозначим через $y_{0}$ единственный на интервале $(-\infty, s-1)$ корень уравнения

$$
\ln c+\psi(s)=\psi(s-1-y), \quad \psi(z)=\frac{d}{d z} \ln \Gamma(z) .
$$

(Здесь Г (z) - гамма-функиия Эйлера.) Тогда:

1) если $y_{0}<\alpha$, то $f(t)$ обладает квазиасимптотикой относительно $\rho(k)$;

2) если $y_{0}>\alpha$, то возможны следующие подслучаи:

2а) если $y_{0}=-1,-2, \ldots$, то существует $c_{1}$ такое, ито $f(t)-c_{1} \delta^{\left(-y_{0}-1\right)}(t)$ обладает квазиасимптотикой относительно $\rho(k)$;

2б) если $y_{0} \neq-1,-2, \ldots$, то, в свою очередь, возможны подслучаи:

$2 \sigma_{1}$ ) если $y_{0}>a$, то $f(t)$ обладает квазиасимптотикой относительно $\rho(k)$;

$\left.2 \sigma_{2}\right)$ если $\alpha<y_{0}<a$, то $f(t)-c_{2} t_{+}^{y_{0}}$ обладает квазиасимптотикой относительно $\rho(k)$ с некоторой постоянной $c_{2}$.

ДокАЗАТЕЛЬСтво. Воспользуемся теоремой 5. Сперва заметим, что в силу конечности порядка $f(t)$ сушествует $Q \geqslant 0$ такое, что $f(t) \in\left(\mathscr{S}_{b, Q}^{a, Q}\right)^{\prime}$. Фиксируя $q=\max \{m, Q+s+1\}$ и полагая $z=r e^{i \beta}$, из (7.19) имеем

$$
\begin{gathered}
\sin ^{q}\left(\frac{\beta}{2}\right) \frac{1}{r \rho(r)} F\left(r e^{i \beta}\right)=\sin ^{q}\left(\frac{\beta}{2}\right) \frac{1}{r \rho(r)}\left(f(t), \frac{\ln \left(c-\frac{t}{r} e^{-i \beta}\right)}{\left(c-\frac{t}{r} e^{-i \beta}\right)^{s}}\right) \\
=\frac{1}{\rho(r)}\left(f(r t), \sin ^{q}\left(\frac{\beta}{2}\right) \frac{\ln \left(c-t e^{-i \beta}\right)}{\left(c-t e^{-i \beta}\right)^{s}}\right)=\frac{1}{\rho(r)}\left(f(r t), \varphi_{0}^{\beta}(t)\right),
\end{gathered}
$$

где $\sin ^{q}(\beta / 2) \frac{\ln \left(c-t e^{-i \beta}\right)}{\left(c-t e^{-i \beta}\right)^{s}}=\varphi_{0}^{\beta}(t), 0<\beta<(0,2 \pi)$.

Покажем, что для чисел $b, a, M=N=Q$ и $\alpha$ семейство функций

$$
\varphi_{0}(t)=\left\{\varphi_{0}^{\beta}(t)=\sin ^{q}\left(\frac{b}{2}\right) \cdot \frac{\ln \left(c-t e^{-i \beta}\right)}{\left(c-t e^{-i \beta}\right)^{s}}, \beta \in J\right\}, \quad J=(0,2 \pi),
$$

допустимо (см. определение 8).

Действительно, для любого $\varepsilon>0$ такого, что $a+\varepsilon<s-1$, и оценки

$$
\mathscr{P}_{b, Q}^{a+\varepsilon, Q}\left[\varphi_{0}^{\beta}(t)\right] \leqslant \frac{\text { const }}{\sin ^{Q+s+1} \frac{\beta}{2}}
$$

где $0<\beta<2 \pi$, следует, что выполнено условие (7.2). Чтобы проверить оставшееся условие (7.3), вычислим преобразование Меллина функций семейства (7.22), 
заметив предварительно, что они аналитичны в комплексной плоскости $\mathbb{C}_{t}$ вне разреза, лежащего на луче, выходящем из начала координат и проходящем через точку $t_{0}=c e^{i \beta}$. Полагая $\operatorname{Im} z>-1$, пользуясь $(2.9)$ и поворачивая луч интегрирования на угол $\beta-\pi$ так, чтобы при этом не задевать разреза, получим

$$
\begin{aligned}
\mathscr{M}\left[\varphi_{0}^{\beta}(t)\right] & =\widehat{\varphi}_{0}^{\beta}(z)=\sin ^{q}\left(\frac{\beta}{2}\right) \int_{0}^{\infty} t^{-i z} \frac{\ln \left(c-t e^{-i \beta}\right)}{\left(c-t e^{-i \beta}\right)^{s}} d t \\
& =\sin ^{q}\left(\frac{\beta}{2}\right) e^{(\beta-\pi)(z+i)} \int_{0}^{\infty} t^{-i z} \frac{\ln (c+t)}{(c+t)^{s}} d t \\
& =\frac{\sin ^{q}(\beta / 2) c^{1-s-i z}}{e^{(\pi-\beta) z} e^{i(\pi-\beta)}[\ln c+\psi(s)-\psi(s-1+i z)] \frac{\Gamma(1-i z) \Gamma(s-1+i z)}{\Gamma(s)}},
\end{aligned}
$$

где $\psi(z)=\frac{d}{d z} \ln \Gamma(z)$. Для последнего интеграла мы использовали табличное значение из [16]. Учитывая, что в полосе $\Pi_{b}^{a+\varepsilon}$ при $|x| \rightarrow+\infty$ выполняется соотношение (6.21), получим, что сушествуют постоянные $C$ и $N$ такие, что при больших $|x|$

$$
|\Gamma(1-i z) \Gamma(s-1+i z)|>C e^{-\pi|x|} \frac{1}{1+|x|^{N}}, \quad z \in \Pi_{b}^{a+\varepsilon} .
$$

Известно, что $\psi(z)=\ln z+O(1 / z),|z| \rightarrow+\infty,|\arg z|<\pi-\varepsilon$. Отсюда и из (7.24) при $z \in \Pi_{b}^{a+\varepsilon}$ вытекает, что существует $R_{0}>0$ такое, что при $|x|>R_{0}$

$$
\left|\frac{\Gamma(1-i z) \Gamma(s-1+i z)}{\Gamma(s)}[\ln c+\psi(s)-\psi(s-1+i z)] c^{1-s-i z}\right| \geqslant \frac{\text { const }}{1+|x|^{N}} e^{-\pi|x|}
$$

Полагая теперь $\beta=1 /|x|$ при $x<-R_{0}$, с учетом (7.24) имеем

$$
\begin{aligned}
\sup _{\beta \in(0,2 \pi)}\left|\widehat{\varphi}_{0}^{\beta}(z)\right| & \geqslant\left|\widehat{\varphi}_{0}^{1 /|x|}(z)\right|>\text { const } \frac{\sin ^{q}(1 / 2|x|)}{1+|x|^{N}} e^{-\pi|x|}\left|e^{(1 /|x|-\pi) z}\right| \\
& \geqslant \frac{\text { const }}{1+|x|^{q+N}} e^{-\pi|x|} e^{x /|x|-\pi x} \geqslant \frac{\text { const }}{1+|x|^{q+N}} .
\end{aligned}
$$

Аналогичная оценка имеет место и при $x>R_{0}$, следует только положить $\beta=2 \pi-$ $1 /|x|$. Таким образом выполнено условие (7.3).

Далее мы изучим общие при всех $\beta \in(0,2 \pi)$ нули семейства $\widehat{\varphi}_{0}(z)$. Из (7.23) вытекает, что нули семейства возможны только при тех $z$, которые удовлетворяют уравнению $\ln c+\psi(s)=\psi(s-1+i z)$. Логарифмируя и дифференцируя известное представление гамма-функции $1 / \Gamma(z)=z e^{z \gamma} \prod_{n=1}^{\infty}(1-z / n) e^{-z / n}$, получим $-\psi(z)=1 / z+\gamma+\sum_{n=1}^{\infty}(1 /(z+n)-1 / n)$. Откуда ${ }^{3}$

$$
\operatorname{Im} \psi(z)=\frac{y}{x^{2}+y^{2}}+\sum_{n=1}^{\infty} \frac{y}{(x+n)^{2}+y^{2}} \neq 0, \text { если } y \neq 0
$$

Следовательно, все корни этого уравнения лежат на чисто мнимой оси. Учитывая монотонность $\psi(\xi)$ при $\xi>0$, выводим, что уравнение $(7.20)$ при $y<s-1$ обладает

\footnotetext{
3 Это рассуждение нам любезно сообщил А. А. Карацуба.
} 
только единственным корнем $y_{0}$. Таким образом, если $\alpha \neq y_{0}$, то семейство (7.22) допустимо.

Из (7.17) и (7.18) выводим, что выполнено условие (7.9) теоремы 5 для семейства $\varphi_{0}(t)$, а потому согласно этой теореме $f(t)$, быть может после вычета контрчлена из пространства $\mathscr{K}_{\alpha}^{a}\left[\varphi_{0}(t)\right]$, определяемого семейством $(7.22)$, обладает квазиасимптотикой относительно $\rho(k)$.

Условие $\left(\frac{d^{j}}{d t^{j}} \varphi_{0}^{\beta}\right)^{(j)}(0)=0$ для $\beta \in(0,2 \pi)$ и какого-то $j \leqslant\langle a\rangle$ эквивалентно условию $(z+i(j+1)) \hat{\varphi}_{0}^{\beta}(z)=0$ при $z=-i(j+1)$ для всех $\beta \in(0,2 \pi)$. А это согласно (7.23) возможно только в случае $2 \mathrm{a})$. В этом случае пространство $\mathscr{K}_{\alpha}^{a}\left[\varphi_{0}(t)\right]$ состоит из функций const $\delta^{(j)}(t)$.

Нам осталось только описать пространство $\mathscr{K}_{\alpha}^{a}\left[\varphi_{0}(t)\right]$ в зависимости от расположения нуля $y_{0}$ преобразования Меллина (7.23) на чисто мнимой оси. Если $y_{0}<\alpha$ или $y_{0}>a$ и $y_{0} \neq-1,-2, \ldots$, то $\mathscr{K}_{\alpha}^{a}\left[\varphi_{0}(t)\right]$ отсутствует. Случай $\alpha<y_{0}<a$ и $y_{0}=-1,-2, \ldots$ соответствует п. 2) замечания 1 к определению 8 . Случай 2б 2 ) соответствует п. 3) того же замечания. Теорема доказана.

ЗАмЕчАнИЕ 1. Если в условиях теоремы 7 вместо $s>0$ считать $s<0$, но $s \neq$ $0,-1,-2, \ldots$, то уравнение $(7.20)$, кроме нуля $y_{0}$, имеет и другие нули $y_{1}, y_{2}, \ldots$, которые монотонно возрастают. Если ни один из них не равен $-1,-2, \ldots$, то теорема 7 остается верной. Если же один из них совпадает с $-1,-2, \ldots$ (а это может случиться только с одним из корней), скажем $y_{j}=-\ell$, где $\ell$ целое положительное, то кроме контрчленов, которые требуют различные случаи теоремы 7 , нужно вычесть еще один контрчлен $c_{3} \delta^{(\ell-1)}(t)$ с некоторой постоянной $c_{3}$.

ЗАмЕчАниЕ 2. Если в условиях теоремы $7 s=0,-1,-2, \ldots$, то вместо $(7.23)$ справедлива формула

$$
\widehat{\varphi}_{0}^{\beta}(z)=\mathrm{const} \sin ^{q}\left(\frac{\beta}{2}\right) e^{i \beta+(\beta-\pi) z} c^{1-s+i z} \Gamma(1-i z) \Gamma(s-1+i z) .
$$

Вместо исследования корней уравнения (7.20) нужно исследовать производные в нуле у функции $(c+t)^{-s} \ln (c+t)$, и если

$$
\left.\frac{d^{j_{\ell}}}{d t^{j \ell}}\left((c+t)^{-s} \ln (c+t)\right)\right|_{t=0}=0, \quad \ell=1,2, \ldots,
$$

то существуют постоянные $c_{\ell}$ такие, что $f(t)-\sum_{\ell} c_{\ell} \delta^{\left(j_{\ell}\right)}(t)$ обладает квазиасимптотикой относительно $\rho(k)$.

Интегральное преобразование с логарифмическим ядром соответствует случаю $s=0$ и $\alpha<a<-1$. Таким образом,

$$
F(z)=\left(f(t), \ln \left(c-\frac{t}{z}\right)\right), \quad z \in \mathbb{C} \backslash[0,+\infty)
$$

Пусть выполнены условия (7.17) и (7.18). Тогда:

$1)$ если $c \neq 1$, то $f(t)$ обладает квазиасимптотикой относительно $\rho(k)$;

2 ) если $c=1$, то сушествует постоянная $c_{1}$ такая, что $f(t)-c_{1} \delta(t)$ обладает квазиасимптотикой относительно $\rho(k)$. 
Интегральное преобразование с ядром арктангенса задается формулой

$$
F(z)=\left(f(t), \operatorname{arctg} \frac{t}{z}\right), \quad z=x+i y, \quad x>0 .
$$

Функция $\operatorname{arctg}(t / z) \in \mathscr{S}_{b}^{a}$ с любыми $-\infty<b<a<-1$ является аналитической функцией параметра $z=x+i y$ в правой полуплоскости $x>0$, а потому для $f(t) \in\left(\mathscr{S}_{b}^{a}\right)^{\prime}$ формула (7.29) определяет там аналитическую функцию.

Пусть $\rho(k)$ - автомодельная функция порядка $\alpha$. Соотношение

$$
\frac{1}{r \rho(r)} F\left(r e^{i \beta}\right)=\frac{1}{r \rho(r)}\left(f(t), \operatorname{arctg} \frac{t}{r} e^{-i \beta}\right)=\frac{1}{\rho(r)}\left(f(r t), \operatorname{arctg}\left(t e^{-i \beta}\right)\right)
$$

и оценка

$$
\mathscr{P}_{b, Q}^{a+\varepsilon, Q}\left[\operatorname{arctg}\left(t e^{-i \beta}\right)\right] \leqslant \frac{\text { const }}{\cos ^{Q} \beta}, \quad|\beta|<\frac{1}{2} \pi,
$$

с $\varepsilon>0$ таким, что $a+\varepsilon<-1$, показывают, что этому интегральному преобразованию соответствует семейство

$$
\varphi_{0}(t)=\left\{\cos ^{Q} \beta \cdot \operatorname{arctg}\left(t e^{-i \beta}\right)=\varphi_{0}^{\beta}(t) \in \mathscr{S}_{b, Q}^{a+\varepsilon, Q}, \beta \in J\right\},
$$

где $J=(-\pi / 2, \pi / 2)$ и $Q \geqslant 0$ - любое целое, $-\infty<b<a<-1$.

Покажем, что для $\alpha<a$ и $\alpha \neq-3,-5,-7, \ldots$ это семейство допустимо.

Действительно, условие (7.2) непосредственно следует из оценки (7.31). Учитывая, что преобразование Меллина

$$
\mathscr{M}\left[\varphi_{0}^{\beta}(t)\right] \equiv \widehat{\varphi}_{0}^{\beta}(z)=\frac{\pi}{2}\left(\cos ^{Q} \beta\right) e^{i \beta(1-i z)} \frac{1}{(i z-1) \sin \left(\frac{\pi}{2} i z\right)}
$$

(см., например, [16]) в полосе $\bar{\Pi}_{\alpha-\varepsilon}^{a+\varepsilon}$ при достаточно больших $x>0$ имеет оценку

$$
\begin{aligned}
\sup _{|\beta|<\frac{\pi}{2}}\left|\widehat{\varphi}_{0}^{\beta}(z)\right| & \geqslant\left|\widehat{\varphi}_{0}^{\pi / 2-1 / x}(z)\right| \geqslant \frac{\text { const }}{1+|x|} \cos ^{Q}\left(\frac{\pi}{2}-\frac{1}{x}\right) e^{(\pi / 2-1 / x) x} e^{-(\pi / 2)|x|} \\
& \geqslant \frac{\text { const }}{1+|x|^{Q+1}}
\end{aligned}
$$

(в этой оценке мы учли, что $\cos (\pi / 2-1 / x) \sim 1 / x$ при $x \rightarrow+\infty$ ) и что аналогичная оценка имеет место и при $x \rightarrow-\infty$, видим, что выполнено и условие (7.3).

Опишем пространство контрчленов $\mathscr{K}_{\alpha}^{a}\left[\varphi_{0}(t)\right]$. Заметим, что при всех $|\beta|<\pi / 2$ сами функции $\varphi_{0}^{\beta}(t)$ и все их производные четного порядка в нуле равны нулю. Кроме того, точки $z_{\tau}=-i(2 \tau+1)$ при $\tau=[\langle a\rangle / 2]+1, \ldots,[\langle\alpha\rangle / 2]$ попадают в полосу $\bar{\Pi}_{\alpha}^{a}$, и согласно (7.33) в них семейства $\left\{\left(z-z_{\tau}\right) \widehat{\varphi}_{0}^{\beta}(z),|\beta|<\pi / 2\right\}$ имеют нули кратности 1. Поэтому

$$
\mathscr{K}_{\alpha}^{a}\left[\varphi_{0}(t)\right]=\sum_{j=0}^{[\langle\alpha\rangle / 2]} c_{j} \delta^{(2 j)}(t),
$$

где $c_{j}$ - произвольные постоянные. 
ТЕОРема 8. Если обобщенная функиия $f(t) \in\left(\mathscr{S}_{b}^{a}\right)^{\prime}, \quad b<a<-1$, обладает квазиасимптотикой относительно автомодельной функиии $\rho(k)$ порядка $\alpha$, $b<\alpha<a, m o:$

1) существует предел

$$
\frac{1}{r \rho(r)} F\left(r e^{i \beta}\right) \underset{r \rightarrow+\infty}{\longrightarrow} c^{\beta} \quad \forall \beta \in\left(-\frac{\pi}{2}, \frac{\pi}{2}\right)
$$

2) существуют $A, m$ и $r_{0}$ такие, что

$$
\frac{1}{r \rho(r)}\left|F\left(r e^{i \beta}\right)\right| \leqslant \frac{A}{\cos ^{m} \beta}, \quad r>r_{0}, \quad \beta \in\left(-\frac{\pi}{2}, \frac{\pi}{2}\right) .
$$

Обратно, пусть выполнень условия (7.35) и (7.36). Тогда существует обобщенная функиия $f_{0}(t)$, принадлежсащая пространству $\mathscr{K}_{\alpha}^{a}\left[\varphi_{0}(t)\right]$, определяемому формулой (7.34), такая, что функиия $f(t)-f_{0}(t)$ обладает квазиасимптотикой относительно $\rho(k)$.

ДокАЗАТЕльСТво. В силу конечностипорядка обобщенная функция $f$ принадлежит пространству $\left(\mathscr{S}_{b, Q}^{a, Q}\right)^{\prime}$ с некоторым $Q \geqslant 0$. Необходимость доказывается точно так же, как и в теореме 6, с использованием соотношений (7.30) и (7.31). Для доказательства же достаточности следует воспользоваться теоремой 5 , учитьвая, что семейство (7.32) допустимо и что из условий (7.35) и (7.36) очевидным образом следует выполнимость условия (7.9) теоремы 5.

\section{§ 8. Приложение}

В этом приложении мы докажем ряд вспомагательных утверждений, которые были использованы в основном тексте.

ЛЕМма ПО. Пусть J - произвольное множсество индексов и задано семейство функиий

$$
\left\{\widehat{\varphi}^{\beta}(z) \in H_{Q}\left(\Pi_{b}^{a}\right), \beta \in I\right\}, \quad \sup _{\beta \in J}\left\|\widehat{\varphi}^{\beta}(z)\right\|_{H_{Q}}<\infty
$$

(см. определение 2), причем найдутся $A$ и $m$ такие, что

$$
\sup _{\beta \in J}\left|\widehat{\varphi}^{\beta}(z)\right| \geqslant \frac{A}{(1+|z|)^{m}} \quad \text { npu } \quad b<\operatorname{Im} z<a
$$

Тогда для любого $\varepsilon>0$ существуют счетное множество $I \subset J$, числа $A_{1}$, $m_{1} u \lambda_{\beta}>0 \quad(\beta \in I)$ такие, что

$$
\sum_{\beta \in I} \lambda_{\beta}<\infty, \quad \sum_{\beta \in I} \lambda_{\beta}\left|\widehat{\varphi}^{\beta}(z)\right| \geqslant \frac{A_{1}}{(1+|z|)^{m_{1}}} \quad \text { npu } \quad b+\varepsilon<\operatorname{Im} z<a-\varepsilon .
$$


ДоКАЗАТЕЛЬСТВо. Если $J$-конечное множество, то лемма тривиальна. Пусть $J$-бесконечное множество и задано $\varepsilon>0$. Отметим, что в условиях леммы $m \geqslant Q$. Кроме того, заметим, что (П.3) достаточно доказать для $|\operatorname{Re} z| \geqslant 1$, ибо эту оценку во всем $\bar{\Pi}_{b+\varepsilon}^{a-\varepsilon}$ можно обеспечить, добавляя, если нужно, к множеству $I$ конечное число элементов множества $J$.

Сушествует постоянная $d>0$, не зависящая от $\beta \in J$, такая, что для любого $z \in \bar{\Pi}_{b+\varepsilon}^{a-\varepsilon} \cap\{|\operatorname{Re} z| \geqslant 1\}$

$$
\left|\widehat{\varphi}^{\beta}(\zeta)\right| \geqslant \frac{1}{2}\left|\widehat{\varphi}^{\beta}(z)\right| \text { при }|\zeta-z|<r_{z, \beta}=\min \left\{\varepsilon, d(1+|z|)^{Q}\left|\widehat{\varphi}^{\beta}(z)\right|\right\} .
$$

Действительно, $\widehat{\varphi}^{\beta}(\zeta)=\widehat{\varphi}^{\beta}(z)+\int_{z}^{\zeta}\left(\widehat{\varphi}^{\beta}\right)^{\prime}(\tau) d \tau$. Учитывая, что

$$
\left|\left(\widehat{\varphi}^{\beta}\right)^{\prime}(z)\right|=\left|\frac{1}{2 \pi i} \int_{|\zeta-z|=\varepsilon} \frac{\widehat{\varphi}^{\beta}(\zeta)}{(\zeta-z)^{2}} d \zeta\right| \leqslant c_{\varepsilon}^{1} \sup _{|\zeta-z|=\varepsilon} \sup _{\beta \in J}\left|\widehat{\varphi}^{\beta}(\zeta)\right| \leqslant \frac{c_{\varepsilon}}{(1+|z|)^{Q}}
$$

где $c_{\varepsilon}$ не зависит от $\beta \in J$ (мы учли (П.1)), получаем

$$
\left|\widehat{\varphi}^{\beta}(\zeta)\right| \geqslant\left|\widehat{\varphi}^{\beta}(z)\right|-|\zeta-z| \frac{c_{\varepsilon}}{(1+|z|)^{Q}} \geqslant \frac{1}{2}\left|\widehat{\varphi}^{\beta}(z)\right|,
$$

если $|\zeta-z| \leqslant\left(1 / 2 c_{\varepsilon}\right)(1+|z|)^{Q}\left|\widehat{\varphi}^{\beta}(z)\right|$.

Разобьем множество $\bar{\Pi}_{b+\varepsilon}^{a-\varepsilon} \cap\{|\operatorname{Re} z| \geqslant 1\}$ на прямоугольники $W_{\ell}$,

$$
W_{\ell}= \begin{cases}\bar{\Pi}_{b+\varepsilon}^{a-\varepsilon} \cap\{\ell \leqslant \operatorname{Re} z \leqslant \ell+1\} & \text { при } \quad \ell=1,2, \ldots, \\ \bar{\Pi}_{b+\varepsilon}^{a-\varepsilon} \cap\{\ell-1 \leqslant \operatorname{Re} z \leqslant \ell\} & \text { при } \quad \ell=-1,-2, \ldots\end{cases}
$$

Пусть $z$ - произвольная точка из $W_{\ell}$. По условию (П.2) существует $\beta_{z} \in J$ такое, что $\left|\hat{\varphi}^{\beta}(z)\right| \geqslant \frac{A}{2(1+|z|)^{m}}$. А потому согласно предыдущему

$$
\left|\widehat{\varphi}^{\beta_{z}}(\zeta)\right| \geqslant \frac{1}{2} \frac{A}{2(1+|z|)^{m}} \geqslant \frac{A_{2}}{(1+|\zeta|)^{m}} \text { при }|\zeta-z|<r_{z, \beta} .
$$

Здесь $A_{2}$ зависит только от $A$ и $m$. Отметим, что

$$
\begin{aligned}
r_{z, \beta} & \geqslant \min \left\{\varepsilon, d(1+|z|)^{Q}\left|\widehat{\varphi}^{\beta_{z}}(z)\right|\right\} \geqslant \min \left\{\varepsilon, d A_{2}(1+|z|)^{Q-m}\right\} \\
& \geqslant \min \left\{\varepsilon, \text { const } \ell^{Q-m}\right\} \geqslant \mathrm{const} \ell^{Q-m} .
\end{aligned}
$$

Теперь всё $W_{\ell}$ оказалось покрытым кругами, радиусы которых $r_{z} \geqslant$ const $\ell^{Q-m}$. По теореме о конечной кратности покрытий (см. [15, с. 33]) из этого покрытия можно выделить конечное подпокрытие с числом элементов, не превосходяшим $n_{\ell}=$ const $\ell^{2(m-Q)}$.

Выбираем такие покрытия в каждом $W_{\ell}$ и соответствуюшие им индексы $\beta_{z}$ нумеруем следующим естественным образом. Сначала нумеруем все элементы из $W_{1}$, затем $W_{-1}$, потом $W_{2}, W_{-2}$ и так далее. Мы получим счетную систему $\beta_{j}$, $j=1,2, \ldots$ 
Полагая теперь $\lambda_{j}=1 / j^{2}$, покажем, что выполнено (П.3). Фиксируем любое $z \in \bar{\Pi}_{b+\varepsilon}^{a-\varepsilon} \cap\{|\operatorname{Re} z| \geqslant 1\}$. Найдется $\ell$ такое, что $z \in W_{\ell}$. Сушествует $j_{0}$ такое, что $z$ попадает в круг, соответствуюший $\beta_{j_{0}}$. Мы имеем

$$
\begin{aligned}
\sum_{j=1}^{\infty} \lambda_{j}\left|\widehat{\varphi}^{\beta_{j}}(z)\right| & \geqslant \lambda_{j_{0}}\left|\widehat{\varphi}^{\beta_{j}}(z)\right| \geqslant \frac{A_{2}}{j_{0}^{2}(1+|z|)^{m}} \geqslant \frac{\text { const }}{(1+|z|)^{m}} \frac{1}{\ell^{2(2 m-2 Q+1)}} \\
& \geqslant \frac{\text { const }}{(1+|z|)^{m}(1+|z|)^{2(2 m-2 Q+1)}} \geqslant \frac{\text { const }}{(1+|z|)^{m_{1}}} .
\end{aligned}
$$

Мы воспользовались тем, что $j_{0} \leqslant \operatorname{const}\left(1+2^{2(m-Q)}+\cdots+\ell^{2(m-Q)}\right) \leqslant$ const $\ell^{2(m-Q)+1}$ и $|z| \sim|\ell|$. Лемма доказана.

ЗАмЕчание 1 . В условиях леммы П0 сушествуют постоянные $A_{2}$ и $m_{2}$ такие, что

$$
\sum_{\beta \in I} \lambda_{\beta}\left|\widehat{\varphi}^{\beta}(z)\right|^{2} \geqslant \frac{A_{2}}{(1+|z|)^{m_{2}}}, \quad z \in \Pi_{b+\varepsilon}^{a-\varepsilon} .
$$

Действительно, пользуясь неравенством Коши-Буняковского, имеем

$$
\frac{A_{2}}{(1+|z|)^{m_{2}}} \leqslant\left(\frac{A_{1}}{(1+|z|)^{m_{1}}}\right)^{2} \leqslant\left(\sum_{\beta \in I} \lambda_{\beta}\left|\widehat{\varphi}^{\beta}(z)\right|\right)^{2} \leqslant \sum_{\beta \in I} \lambda_{\beta} \sum_{\beta \in I} \lambda_{\beta}\left|\widehat{\varphi}^{\beta}(z)\right|^{2} .
$$

Отсюда и из (П.3) вытекает (П.4).

ЗАмЕчАниЕ 2 . Если $J$ - несчетное множество, то из леммы следует, что оценка (П.2), быть может с другим $m$, выполняется для счетного множества индексов $I \subset J$. Это непосредственно вытекает из оценки (П.3). Очевидно также, что если $J$ - счетное множество, то в качестве $I$ можно брать само множество $J$.

Следуюшая лемма является некоторым вариантом классической теоремы Карлесона о короне. Мы приводим ее в том виде, который нам был нужен в основном тексте. Идеи доказательства достаточно традищионны и содержатся, например, в [20] (см. также цитируемую там литературу).

Лемма П1. Пусть I - счетное множество и задано семейство функций

$$
\left\{\varphi^{\beta}(z) \in H_{L}\left(\Pi_{b}^{a}\right), \beta \in I\right\} ; \quad \sup _{\beta \in I}\left\|\varphi^{\beta}(z)\right\|_{H_{L}}<\infty .
$$

(Как обычно, $a>b$ - нечелые вещественные числа.) Пусть заданы $\lambda_{\beta}>0$, $\sum_{\beta \in I} \lambda_{\beta}<\infty$, такие, что вьполнена оценка

$$
\sum_{\beta \in I} \lambda_{\beta}\left|\varphi^{\beta}(z)\right|^{2} \geqslant \frac{A}{(1+|z|)^{m}}, \quad z \in \Pi_{b}^{a}
$$

для некоторых постоянных А и $m$, причем для любой попавшей в полосу $\Pi_{b}^{a}$ точки $z_{\ell}=-i \ell(\ell-$ челое положительное, $-a<\ell<-b)$ существует $\beta_{\ell} \in I$ maкое, что

$$
\operatorname{Res}_{z=z_{\ell}} \varphi^{\beta_{\ell}}(z) \neq 0
$$


Тогда для любого сколь угодно малого $\varepsilon>0$ существует число $Q$ и семейство аналитических в $\Pi_{b+\varepsilon}^{a-\varepsilon}$ функиий $\left\{g_{\beta}(z) \in H_{Q}\left(\Pi_{b+\varepsilon}^{a-\varepsilon}\right), \beta \in I\right\}$ таких, что

$$
\begin{gathered}
\sup _{\beta \in I}\left\|g_{\beta}(z)\right\|_{H_{Q}}<\infty, \\
\sum_{\beta \in I} \lambda_{\beta} g_{\beta}(z) \varphi^{\beta}(z)=1, \quad z \in \Pi_{b+\varepsilon}^{a-\varepsilon}, \\
g_{\beta}\left(z_{\ell}\right)=0 \quad \forall \beta \in I, \quad z_{\ell}=-i \ell, \quad \ell=1,2, \ldots, \quad z_{\ell} \in \Pi_{b}^{a} .
\end{gathered}
$$

ДокАЗАТЕЛЬСТво. Фиксируем достаточно малое $\varepsilon>0$ такое, чтобы $a-\varepsilon>$ $b+\varepsilon$,

$$
\left\{z_{\ell}=-i \ell, \ell=1,2, \ldots, z_{\ell} \in \Pi_{b}^{a}\right\}=\left\{z_{\ell}=-i \ell, \ell=1,2, \ldots, z_{\ell} \in \Pi_{b+\varepsilon}^{a-\varepsilon}\right\},
$$

и полагаем $f_{\beta}(z)=\Theta_{b}^{a}(z) \varphi^{\beta}(z), \beta \in I$ (см. определение 2).

Семейство $\left\{f_{\beta}(z) \in H_{L-r}\left(\Pi_{b}^{a}\right), \beta \in I\right\}$ принадлежит ${ }^{I} H_{L-r}\left(\Pi_{b}^{a}\right)$, т.е. ограничено по $\beta \in I$ и состоит из аналитических в $\Pi_{b}^{a}$ функций. Рассмотрим семейство функций

$$
\psi_{\beta}(z, \bar{z})=\frac{\bar{f}_{\beta}(z)}{\sum_{\beta \in I}\left|f_{\beta}(z)\right|^{2} \lambda_{\beta}}, \quad \beta \in I .
$$

Очевидно,

$$
\sum_{\beta \in I} f_{\beta}(z) \psi_{\beta}(z, \bar{z}) \lambda_{\beta} \equiv 1
$$

Заметим, что

$$
\sup _{\beta \in I} \sup _{z \in \Pi_{b+\varepsilon / 2}^{a-\varepsilon / 2}}\left|(1+|z|)^{q_{j}}\left(\frac{\partial}{\partial \bar{z}}\right)^{j} \psi_{\beta}(z, \bar{z})\right|<\infty, \quad j=0,1, \ldots,
$$

для некоторых $q_{j}$.

Рассмотрим множество функций $G_{\alpha \beta}(z, \bar{z})=\psi_{\alpha}(z, \bar{z}) \frac{\partial}{\partial \bar{z}} \psi_{\beta}(z, \bar{z}), \alpha, \beta \in I$. В силу (П.13) существуют постоянные $c$ и $Q_{1}$, не зависящие от $\beta \in I$ и такие, что

$$
\left|G_{\alpha \beta}(z, \bar{z})\right| \leqslant c(1+|z|)^{Q_{1}}, \quad \alpha, \beta \in I, \quad z \in \Pi_{b+1 / 2 \varepsilon}^{a-1 / 2 \varepsilon} .
$$

Пусть $N>Q_{1}+1$ и $c$ настолько велико, что $c+z^{2 N}$ не обращается в нуль в полосе $\Pi_{b}^{a}$. Положим

$$
b_{\alpha \beta}(z, \bar{z})=\frac{c+z^{2 N}}{2 \pi i} \int_{\Pi_{b+\varepsilon / 2}^{a-\varepsilon / 2}} \frac{G_{\alpha \beta}(\zeta, \bar{\zeta})}{c+\zeta^{2 N}} \frac{1}{\zeta-z} d \xi d \eta, \quad \zeta=\xi+i \eta
$$

Очевидно, что $b_{\alpha \beta}(z, \bar{z})$ являются решениями $\bar{\partial}$-задачи

$$
\frac{\partial}{\partial \bar{z}} b_{\alpha \beta}(z, \bar{z})=G_{\alpha \beta}(z, \bar{z}), \quad \alpha, \beta \in I, \quad z \in \Pi_{b+\varepsilon / 2}^{a-\varepsilon / 2} .
$$


В силу (П.14) и (П.15) сушествуют $M_{1}, M_{2}, n_{1}, n_{2}$, не зависящие от $\alpha, \beta \in I$ и такие, что

$$
\left|b_{\alpha \beta}(z, \bar{z})\right| \leqslant M_{1}(1+|z|)^{n_{1}}, \quad\left|\frac{\partial}{\partial \bar{z}} b_{\alpha \beta}(z, \bar{z})\right| \leqslant M_{2}(1+|z|)^{n_{2}}, \quad z \in \Pi_{b+\varepsilon}^{a-\varepsilon} .
$$

Полагаем теперь

$$
g_{\beta}^{1}(z)=\psi_{\beta}(z, \bar{z})+\sum_{\alpha \in I}\left[b_{\beta \alpha}(z, \bar{z})-b_{\alpha \beta}(z, \bar{z})\right] f_{\alpha}(z) \lambda_{\alpha}, \quad z \in \Pi_{b+\varepsilon}^{a-\varepsilon} .
$$

В силу (П.6), (П.14), (П.17) сушествуют $M_{3}, n_{3}$, не зависяшие от $\beta \in I$, такие, что

$$
\left|g_{\beta}^{1}(z)\right| \leqslant M_{3}(1+|z|)^{n_{3}}, \quad z \in \Pi_{b+\varepsilon}^{a-\varepsilon} .
$$

Из (П.18) с учетом (П.12) получаем

$$
\begin{aligned}
\sum_{\beta \in I} g_{\beta}^{1}(z) f_{\beta}(z) \lambda_{\beta}= & \sum_{\beta \in I} \psi_{\beta}(z, \bar{z}) f_{\beta}(z) \lambda_{\beta} \\
& +\sum_{\beta \in I} \sum_{\alpha \in I}\left[b_{\beta \alpha}(z, \bar{z})-b_{\alpha \beta}(z, \bar{z})\right] f_{\alpha}(z) f_{\beta}(z) \lambda_{\alpha} \lambda_{\beta} \\
= & \sum_{\beta \in I} \psi_{\beta}(z, \bar{z}) f_{\beta}(z) \lambda_{\beta} \equiv 1, \quad z \in \Pi_{b+\varepsilon}^{a-\varepsilon}
\end{aligned}
$$

Проверим, что $g_{\beta}^{1}(z)$ аналитичны в полосе $\Pi_{b+\varepsilon}^{a-\varepsilon}$. Учитывая (П.16) и тот факт, что производную по $\bar{z}$ в силу оценок (П.14) и (П.17) можно вынести за знак суммы, дифференцируя (П.18), имеем

$$
\begin{aligned}
\frac{\partial}{\partial \bar{z}} g_{\beta}^{1}(z)= & \frac{\partial}{\partial \bar{z}} \psi_{\beta}(z, \bar{z})+\frac{\partial}{\partial \bar{z}} \sum_{\alpha \in I}\left[b_{\beta \alpha}(z, \bar{z})-b_{\alpha \beta}(z, \bar{z})\right] f_{\alpha}(z) \lambda_{\alpha} \\
= & \frac{\partial}{\partial \bar{z}} \psi_{\beta}(z, \bar{z})+\sum_{\alpha \in I} \frac{\partial b_{\beta \alpha}(z, \bar{z})}{\partial \bar{z}} f_{\alpha}(z) \lambda_{\alpha}-\sum_{\alpha \in I} \frac{\partial b_{\alpha \beta}(z, \bar{z})}{\partial \bar{z}} f_{\alpha}(z) \lambda_{\alpha} \\
= & \frac{\partial}{\partial \bar{z}} \psi_{\beta}(z, \bar{z})+\sum_{\alpha \in I} \varphi_{\beta}(z, \bar{z}) \frac{\partial \psi_{\alpha}(z, \bar{z})}{\partial \bar{z}} f_{\alpha}(z) \lambda_{\alpha} \\
& -\sum_{\alpha \in I} \psi_{\alpha}(z, \bar{z}) \frac{\partial \varphi_{\beta}(z, \bar{z})}{\partial \bar{z}} f_{\alpha}(z) \lambda_{\alpha} \\
= & \frac{\partial}{\partial \bar{z}} \psi_{\beta}(z, \bar{z})+\psi_{\beta}(z, \bar{z}) \frac{\partial}{\partial \bar{z}} \sum_{\alpha \in I} \psi_{\alpha}(z, \bar{z}) f_{\alpha}(z) \lambda_{\alpha} \\
& -\frac{\partial}{\partial \bar{z}} \psi_{\beta}(z, \bar{z}) \sum_{\alpha \in I} \psi_{\alpha}(z, \bar{z}) f_{\alpha}(z) \lambda_{\alpha}=0 .
\end{aligned}
$$

Мы учли (П.12) и аналитичность функций $f_{\alpha}(z)$.

Семейство $\left\{g_{\beta}(z)=g_{\beta}^{1}(z) \Theta_{b}^{a}(z), \beta \in I\right\}$ в силу (П.17) и (П.19) будет искомым семейством функций. Лемма доказана.

$$
\begin{gathered}
\text { Пусть теперь } \omega(t) \in \underset{0}{\mathscr{S}_{b, M}^{a, M}, \text { a } \varphi_{\lambda}(t) \in F^{M}\left[\stackrel{0}{\mathscr{J}}_{b, N}^{a, N}\right] . \text { Рассмотрим функцию }} \\
\psi \psi\left[\omega, \varphi_{\lambda}\right](t)=\int_{0}^{\infty} \omega(\varkappa) \varphi_{\varkappa}\left(\frac{t}{\varkappa}\right) \frac{d \varkappa}{\varkappa} .
\end{gathered}
$$


ЛЕмМА П2. Билинейное отображение

$$
\underset{0}{\mathscr{S}_{b, M}^{a, M}} \times F^{M}\left[\begin{array}{c}
0 \\
\mathscr{S}_{b, N}^{a, N}
\end{array}\right] \stackrel{\psi}{\longrightarrow} \stackrel{0}{\mathscr{S}}_{b, M+N}^{a, M+N}
$$

корректно определено и непрерывно.

ДокаЗАТЕльство. Сначала докажем лемму для случая $M=N=0$. При каждом $\lambda>0$, так как $\varphi_{\lambda}(t) \in \stackrel{0}{\mathscr{S}}_{b, 0}^{a, 0}$, имеем

$$
\varphi_{\lambda}(t)=\sum_{j=\langle a\rangle+1}^{\langle b\rangle} c_{j}(\lambda) t^{j}+\chi_{\lambda}(t), \quad t^{b} \chi_{\lambda}(t) \in L_{1}(0,1), \quad t^{a} \varphi_{\lambda}(t) \in L_{1}(1, \infty)
$$

Отсюда и из (П.20) имеем (пока формально)

$$
\begin{aligned}
\psi\left[\omega, \varphi_{\lambda}\right](t) & =\int_{0}^{\infty} \omega(\varkappa) \varphi_{\varkappa}\left(\frac{t}{\varkappa}\right) \frac{d \varkappa}{\varkappa} \\
& =\int_{0}^{\infty} \omega(\varkappa) \chi_{\varkappa}\left(\frac{t}{\varkappa}\right) \frac{d \varkappa}{\varkappa}+\sum_{j=\langle a\rangle+1}^{\langle b\rangle} \int_{0}^{\infty} c_{j}(\varkappa) \omega(\varkappa) \frac{d \varkappa}{\varkappa^{j+1}} t^{j} \\
& =\psi\left[\omega, \chi_{\lambda}\right](t)+\sum_{j=\langle a\rangle+1}^{\langle b\rangle} c_{j} t^{j}
\end{aligned}
$$

где $c_{j}=\int_{0}^{\infty} c_{j}(\varkappa) \omega(\varkappa) \frac{d \varkappa}{\varkappa^{j+1}}$. Для строгого доказательства (П.23) достаточно показать, что все интегралы, фигурирующие там, корректно определены. Оценим сначала интегралы, определяюшие постоянные $c_{j}, j=\langle a\rangle+1, \ldots,\langle b\rangle$ :

$$
\begin{aligned}
\int_{0}^{\infty}\left|c_{j}(\varkappa) \omega(\varkappa)\right| \frac{d \varkappa}{\varkappa} & \leqslant \sup _{\varkappa>0}\left|c_{j}(\varkappa)\right| \int|\omega(\varkappa)| \frac{d \varkappa}{\varkappa^{j+1}} \\
& \leqslant \mathscr{P}_{(b, 0)}^{0(a, 0)}\left[\varphi_{\lambda}(t)\right]\left\{\int_{0}^{1} \frac{|\omega(\varkappa)|}{\varkappa^{\langle b\rangle+1}} d \varkappa+\int_{1}^{\infty} \frac{|\omega(\varkappa)|}{\left.\varkappa^{\langle a\rangle+2} d \varkappa\right\}}\right. \\
& \leqslant \mathscr{P}_{(b, 0)}^{0(a, 0)}\left[\varphi_{\lambda}(t)\right]\left\{\int_{0}^{1} \varkappa^{b}|\omega(\varkappa)| d \varkappa+\int_{1}^{\infty} \varkappa^{a}|\omega(\varkappa)| d \varkappa\right\} \\
& \leqslant \mathscr{P}_{(b, 0)}^{0(a, 0)}\left[\varphi_{\lambda}(t)\right] \cdot \mathscr{P}_{b, 0}^{a, 0}[\omega] .
\end{aligned}
$$

Здесь учтены соотношения (1.2).

Рассмотрим теперь первое слагаемое в правой части формулы (П.23). Имеем

$$
\begin{aligned}
& \int_{0}^{1} t^{b}\left|\psi\left[\omega, \chi_{\lambda}\right](t)\right| d t=\int_{0}^{1} t^{b}\left|\int_{0}^{\infty} \omega(\varkappa) \chi_{\varkappa}\left(\frac{t}{\varkappa}\right) \frac{d \varkappa}{\varkappa}\right| d t \\
& \leqslant \int_{0}^{\infty}|\omega(\varkappa)| \int_{0}^{1} t^{b}\left|\chi_{\varkappa}\left(\frac{t}{\varkappa}\right)\right| d t \frac{d \varkappa}{\varkappa}=\int_{0}^{\infty} \varkappa^{b+1}|\omega(\varkappa)| \frac{d \varkappa}{\varkappa} \int_{0}^{1 / \varkappa} t^{b}\left|\chi_{\varkappa}(t)\right| d t
\end{aligned}
$$




$$
\begin{aligned}
\leqslant & \int_{0}^{1} \varkappa^{b}|\omega(\varkappa)| d \varkappa\left\{\int_{0}^{1}+\int_{1}^{1 / \varkappa}\right\} t^{b}\left|\chi_{\varkappa}(t)\right| d t \\
& +\int_{1}^{\infty} \varkappa^{b}|\omega(\varkappa)| d \varkappa \int_{0}^{1 / \varkappa} t^{b}\left|\chi_{\varkappa}(t)\right| d t \\
\leqslant & \int_{0}^{1} \varkappa^{b}|\omega(\varkappa)| d \varkappa \cdot \sup _{\varkappa>0} \int_{0}^{1} t^{b}\left|\chi_{\varkappa}(t)\right| d t+\int_{0}^{1} \varkappa^{b}|\omega(\varkappa)| d \varkappa \int_{1}^{1 / \varkappa} t^{b}\left|\chi_{\varkappa}(t)\right| d t \\
& +\int_{1}^{\infty} \varkappa^{a}|\omega(\varkappa)| d \varkappa \cdot \sup _{\varkappa>0} \int_{0}^{1} t^{b}\left|\chi_{\varkappa}(t)\right| d t .
\end{aligned}
$$

Первое и последнее слагаемые в правой части неравенства (П.25) оцениваются произведением норм $\mathscr{P}_{b, 0}^{a, 0}[\omega(t)] \cdot \mathscr{P}_{(b, 0)}^{0(a, 0)}\left[\varphi_{\lambda}(t)\right]$. Оценим среднее слагаемое. Имеем

$$
\begin{aligned}
& \int_{0}^{1} \varkappa^{b}|\omega(\varkappa)| d \varkappa \int_{1}^{1 / \varkappa} t^{b}\left|\chi_{\varkappa}(t)\right| d t \leqslant \\
& \leqslant \int_{0}^{1} \varkappa^{b}|\omega(\varkappa)| d \varkappa \int_{1}^{1 / \varkappa} t^{b}\left|\varphi_{\varkappa}(t)-\sum_{j=\langle a\rangle+1}^{\langle b\rangle} c_{j}(\varkappa) t^{j}\right| d t \\
& \leqslant \int_{0}^{1} \varkappa^{b}|\omega(\varkappa)| d \varkappa \int_{1}^{1 / \varkappa} t^{b}\left|\varphi_{\varkappa}(t)\right| d t \\
&+\int_{0}^{1} \varkappa^{b}|\omega(\varkappa)| d \varkappa \int_{1}^{1 / \varkappa} \sum_{j=\langle a\rangle+1}^{\langle b\rangle}\left|c_{j}(\varkappa)\right| t^{b+j} d t \\
& \leqslant \int_{0}^{1} \varkappa^{b}|\omega(\varkappa)| d \varkappa \cdot \sup _{\varkappa>0}^{\infty} t_{1}^{a}\left|\varphi_{\varkappa}(t)\right| d t \\
&+\sup _{\varkappa>0} \sum_{j=\langle a\rangle+1}^{\langle b\rangle}\left|c_{j}(\varkappa)\right| \cdot \int_{0}^{1} \varkappa^{b}|\omega(\varkappa)| d \varkappa \int_{1}^{1 / \varkappa} t^{b+\langle b\rangle} d t \\
& \leqslant \mathscr{P}_{b, 0}^{a, 0}[\omega(t)] \cdot \mathscr{P}_{(b, 0)}^{0(a, 0)}\left[\varphi_{\lambda}(t)\right] \\
&+\mathscr{P}_{(b, 0)}^{0(a, 0)}\left[\varphi_{\lambda}(t)\right] \frac{1}{|b+\langle b\rangle+1|} \int_{0}^{1} \varkappa^{b}|\omega(\varkappa)| \frac{\varkappa^{b+\langle b\rangle+1}}{\varkappa^{b+}} d \varkappa \\
& \leqslant \operatorname{const}_{\mathscr{P}^{a, 0}[\omega(t)] \cdot \mathscr{P}_{(b, 0)}^{0(a, 0)}\left[\varphi_{\lambda}(t)\right] .}
\end{aligned}
$$

Из оценок слагаемых (П.25) с учетом (П.26) вытекает оценка

$$
\int_{0}^{1} t^{b}\left|\psi\left[\omega, \chi_{\lambda}\right](t)\right| d t \leqslant \text { const } \cdot \mathscr{P}_{b, 0}^{a, 0}[\omega(t)] \cdot \mathscr{P}_{(b, 0)}^{0(a, 0)}\left[\varphi_{\lambda}(t)\right]
$$

Точно так же можно показать, что справедлива аналогичная оценка для $\int_{0}^{B} t^{b}\left|\psi\left[\omega, \chi_{\lambda}\right](t)\right| d t$ с любым $B>1$ и постоянной, зависящей от $B$. Следовательно, по теореме Фубини $\int_{0}^{\infty} \omega(\varkappa) \chi \varkappa(t / \varkappa) d \varkappa / \varkappa$ сходится при почти всех $t$. 
Далее, имеем

$$
\begin{aligned}
\int_{1}^{\infty} t^{a} \mid & \psi\left[\omega, \varphi_{\lambda}\right](t)\left|d t=\int_{1}^{\infty} t^{a}\right| \int_{0}^{\infty} \omega(\varkappa) \varphi_{\varkappa}\left(\frac{t}{\varkappa}\right) \frac{d \varkappa}{\varkappa} \mid d t \\
\leqslant & \int_{0}^{\infty} \varkappa^{a+1}|\omega(\varkappa)| \frac{d \varkappa}{\varkappa} \int_{1 / \varkappa}^{\infty} t^{a}\left|\varphi_{\varkappa}(t)\right| d t \\
= & \int_{0}^{1} \varkappa^{a}|\omega(\varkappa)| d \varkappa \int_{1 / \varkappa}^{\infty} t^{a}\left|\varphi_{\varkappa}(t)\right| d t+\int_{1}^{\infty} \varkappa^{a}|\omega(\varkappa)| d \varkappa \int_{1 / \varkappa}^{1} t^{a}\left|\varphi_{\varkappa}(t)\right| d t \\
& +\int_{1}^{\infty} \varkappa^{a}|\omega(\varkappa)| d \varkappa \int_{1}^{\infty} t^{a}\left|\varphi_{\varkappa}(t)\right| d t .
\end{aligned}
$$

Первое и последнее слагаемые справа в (П.28) непосредственно оцениваются произведением норм $\mathscr{P}_{b, 0}^{a, 0}[\omega(t)] \cdot \mathscr{P}_{(b, 0)}^{0(a, 0)}\left[\varphi_{\lambda}(t)\right]$. Оценим среднее слагаемое. Имеем

$$
\begin{aligned}
& \int_{1}^{\infty} \varkappa^{a}|\omega(\varkappa)| d \varkappa \int_{1 / \varkappa}^{1} t^{a}\left|\chi_{\varkappa}(t)+\sum_{j=\langle a\rangle+1}^{\langle b\rangle} c_{j}(\varkappa) t^{j}\right| d t \\
& \leqslant \int_{1}^{\infty} \varkappa^{a}|\omega(\varkappa)| d \varkappa \int_{1 / \varkappa}^{1} t^{a}\left|\chi_{\varkappa}(t)\right| d t \\
&+\int_{1}^{\infty} \varkappa^{a}|\omega(\varkappa)| d \varkappa \sum_{j=\langle a\rangle+1}^{\langle b\rangle}\left|c_{j}(\varkappa)\right| \int_{1 / \varkappa}^{1} t^{a+j} d t \\
& \leqslant \int_{1}^{\infty} \varkappa^{a}|\omega(\varkappa)| d \varkappa \cdot \sup _{\varkappa>0} \int_{0}^{1} t^{b}\left|\chi_{\varkappa}(t)\right| d t \\
&+\sup _{\varkappa>0} \sum_{j=\langle a\rangle+1}^{\langle b\rangle}\left|c_{j}(\varkappa)\right| \cdot \int_{1}^{\infty} \varkappa^{a}|\omega(\varkappa)| d \varkappa \int_{1 / \varkappa}^{1} t^{a+j} d t \\
& \leqslant \mathscr{P}_{b, 0}^{a, 0}[\omega(t)] \cdot \mathscr{P}_{(b, 0)}^{0(a, 0)}\left[\varphi_{\lambda}(t)\right] \\
&+\mathscr{P}_{(b, 0)}^{0(a, 0)}\left[\varphi_{\lambda}(t)\right] \frac{1}{|a+\langle a\rangle+2|} \int_{1}^{\infty}|\omega(\varkappa)| \frac{d \varkappa}{\varkappa^{\langle a\rangle+2}} \\
& \leqslant \operatorname{const} \cdot \mathscr{P}_{b, 0}^{a, 0}[\omega(t)] \cdot \mathscr{P}_{(b, 0)}^{0(a, 0)}\left[\varphi_{\lambda}(t)\right] .
\end{aligned}
$$

Из (П.28) с учетом оценок (П.29) получаем

$$
\int_{1}^{\infty} t^{a}\left|\psi\left[\omega, \varphi_{\lambda}\right](t)\right| d t \leqslant \mathrm{const} \cdot \mathscr{P}_{b, 0}^{a, 0}[\omega(t)] \cdot \mathscr{P}_{(b, 0)}^{0(a, 0)}\left[\varphi_{\lambda}(t)\right] .
$$

Мы доказали, что интеграл в (П.20) абсолютно сходится и функция $\psi$ принадлежит $L_{1}^{\mathrm{loc}}(0,+\infty)$. Теперь из $(П .23),(\Pi .24),(\Pi .27)$ и $(П .30)$ следует

$$
\mathscr{P}_{b, 0}^{a, 0}\left[\psi\left[\omega, \varphi_{\lambda}\right](t)\right] \leqslant \mathrm{const} \cdot \mathscr{P}_{b, 0}^{a, 0}[\omega] \cdot \mathscr{P}_{(b, 0)}^{0(a, 0)}\left[\varphi_{\lambda}\right],
$$

т.е. следует утверждение леммы для $M=N=0$.

Доказательство леммы (по индукции) вытекает из следующих легко проверяемых замечаний. 
ЗАмЕчАНИЕ П1. Пусть $\varphi_{\lambda}(t) \in F^{0}\left[\begin{array}{c}0 \\ \mathscr{S}^{a}, N \\ b, N\end{array}\right], N \geqslant 1$; тогда при $t>0$ функция $\psi(t)$, определяемая формулой (П.20), имеет обобщенную производную, причем

$$
t \frac{d}{d t} \psi(t)=\int_{0}^{\infty} \omega(\varkappa) \frac{t}{\varkappa} \varphi_{\varkappa}^{\prime}\left(\frac{t}{\varkappa}\right) \frac{d \varkappa}{\varkappa}
$$

Операция

$$
t \frac{d}{d t}: F^{q}\left[\begin{array}{c}
0 \\
\mathscr{S}_{b, N}^{a, N}
\end{array}\right] \longrightarrow F^{q}\left[\begin{array}{c}
0 \\
\mathscr{S}_{b, N-1}^{a, N-1}
\end{array}\right]
$$

корректно определена и непрерьвна.

ЗАмечание П2. Пусть $\varphi_{\lambda}(t) \in F^{M}\left[\begin{array}{c}0 \\ \mathscr{S}_{b, N}^{a, N}\end{array}\right]$ и $\omega(t) \in \underset{0}{\mathscr{S}_{b, M}^{a, M}}, M \geqslant 1$. Тогда при $t>0$ функция $\psi(t)$, определяемая формулой (П.20), имеет обобщенную производную, причем

$$
t \frac{d}{d t} \psi(t)=\left.\int_{0}^{\infty} \lambda \frac{d}{d \lambda} \varphi_{\lambda}(\varkappa)\right|_{\lambda=t / \varkappa} \omega\left(\frac{t}{\varkappa}\right) \frac{d \varkappa}{\varkappa}+\int_{0}^{\infty} \varphi_{t / \varkappa}(\varkappa) \frac{t}{\varkappa} \omega^{\prime}\left(\frac{t}{\varkappa}\right) \frac{d \varkappa}{\varkappa} .
$$

Операции

$$
\lambda \frac{d}{d \lambda}: F^{M}\left[\begin{array}{c}
0 \\
\mathscr{S}_{b, N}^{a, N}
\end{array}\right] \longrightarrow F^{M-1}\left[\begin{array}{c}
0 \\
\mathscr{S}_{b, N}^{a, N}
\end{array}\right] \quad \text { и } t \frac{d}{d t}: \mathscr{S}_{0}^{a, M} \underset{b, M}{\longrightarrow} \mathscr{S}_{0}^{a, M-1}
$$

корректно определены и непрерывны.

Лемма доказана.

ЛЕмма П3. Пусть $I$ - счетное множсество и семейство функиий $\left\{\omega^{\beta}(t) \in\right.$ $\left.\mathscr{S}_{0}^{a, M_{1}}, \beta \in I\right\} \equiv \omega(t)$ принадлежит $\underset{I_{0}}{\mathscr{S}_{b, M_{1}}^{a}, M_{1}}$. Тогда для любого $d \in(b, a) u$ $\sigma>0$ таких, что $d-\sigma \geqslant b$, справедлива оценка

$$
{ }_{I} \mathscr{P}_{d, M_{1}}^{a, M_{1}}\left[\frac{1}{t^{\sigma}} \omega(t)\right] \leqslant \mathrm{const} \cdot{ }_{I} \mathscr{P}_{d-\sigma, M_{1}}^{a-\sigma, M_{1}}[\omega(t)] \leqslant \text { const } \cdot_{I} \mathscr{P}_{d-\sigma, M_{1}}^{a, M_{1}}[\omega(t)]
$$

ДокАЗАТЕЛЬСтво. Так как

$$
\left|\left(t \frac{d}{d t}\right)^{j} \frac{1}{t^{\sigma}} \omega^{\beta}(t)\right| \leqslant \frac{1}{t^{\sigma}} \sum_{\ell=0}^{j} c_{\ell}\left|\left(t \frac{d}{d t}\right)^{\ell} \omega^{\beta}(t)\right|
$$

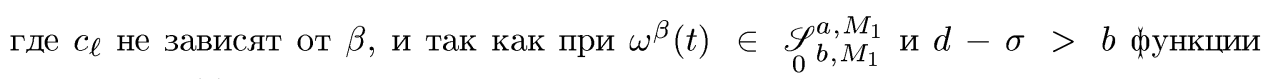
$t^{d-\sigma}\left|t^{j}\left(\omega^{\beta}\right)^{(j)}\right|$ принадлежат $L_{1}(0, \delta)$ для $j=0,1, \ldots, M_{1}$, то согласно $(2.3)$ 


$$
\begin{aligned}
& T_{\left(1 / t^{\sigma}\right) \omega^{\beta}}^{\langle d-\sigma\rangle}(t) \equiv 0 . \text { Имеем } \\
& \mathscr{P}_{d, M_{1}}^{a, M_{1}}\left[\frac{1}{t^{\sigma}} \omega^{\beta}(t)\right]=\max _{j \leqslant M_{1}} \int_{0}^{1} t^{d}\left|\left(t \frac{d}{d t}\right)^{j} \frac{1}{t^{\sigma}} \omega^{\beta}(t)\right| d t \\
& +\max _{j \leqslant M_{1}} \int_{1}^{\infty} t^{a}\left|\left(t \frac{d}{d t}\right)^{j} \frac{1}{t^{\sigma}} \omega^{\beta}(t)\right| d t \\
& \leqslant \max _{j \leqslant M_{1}} \sum_{\ell=0}^{j} c_{\ell}\left\{\int_{0}^{1} t^{d-\sigma}\left|\left(t \frac{d}{d t}\right)^{\ell} \frac{1}{t^{\sigma}} \omega^{\beta}(t)\right| d t\right. \\
& \left.+\int_{1}^{\infty} t^{a-\sigma}\left|\left(t \frac{d}{d t}\right)^{\ell} \frac{1}{t^{\sigma}} \omega^{\beta}(t)\right| d t\right\} \\
& \leqslant \text { const } \cdot \mathscr{P}_{d-\sigma, M_{1}}^{a-\sigma, M_{1}}\left[\omega^{\beta}(t)\right] .
\end{aligned}
$$

Откуда и следует оценка (П.36). Лемма доказана.

ЛЕмма П4. Пусть семейство последовательностей функиий

$$
\left\{\varphi_{\lambda}^{\beta}(t) \in \mathscr{S}_{b, N, \delta}^{a, M}, \beta \in I, \lambda>0\right\} \equiv \varphi_{\lambda}(t) \in{ }^{I} F^{q}\left[\mathscr{S}_{b, N, \delta}^{a, M}\right],
$$

где $b<a, M \leqslant N u q \geqslant 0$. Пусть, кроме того,

$$
\lambda^{\sigma} \varphi_{\lambda}(t) \in{ }^{I} F^{q}\left[\mathscr{S}_{d, M}^{a, M}\right]
$$

для некоторых $\sigma>0$ u $d \in(b, a)$ maких, что $d-\sigma>b$. Тогда существует постоянная с такая, что для любого $M_{1} \leqslant q$ илюбого семейства $\omega \in{ }_{I}^{\mathscr{S}_{0}^{a, M_{1}}}{ }_{d-\sigma, M_{1}}$

$$
\mathscr{P}_{d, M_{1}+M}^{a, M_{1}+M}\left[B_{k} \omega\right] \leqslant \frac{c}{k^{\sigma}} \cdot{ }^{I} \mathscr{P}_{(d, M)}^{M_{1}(a, M)}\left[\lambda^{\sigma} \varphi_{\lambda}(t)\right] \cdot{ }_{I} \mathscr{P}_{d-\sigma, M_{1}}^{a, M_{1}}[\omega] .
$$

Здесь семейство операторов $B_{k}$ построено по семейству последовательносmей $\varphi_{\lambda}(t)(c \mathcal{M} .(3.16))$.

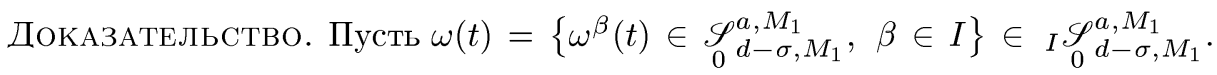
Имеем

$$
\begin{aligned}
B_{k} \omega & =\sum_{\beta \in I} \int_{0}^{\infty} \omega^{\beta}(\varkappa)_{k \varkappa}^{0}\left(\frac{t}{\varkappa}\right) \frac{d \varkappa}{\varkappa} \\
& =\frac{1}{k^{\sigma}} \sum_{\beta \in I} \int_{0}^{\infty} \frac{1}{\varkappa^{\sigma}} \omega^{\beta}(\varkappa)(k \varkappa)^{\sigma} \stackrel{\varphi}{\varphi}_{k \varkappa}^{\beta}\left(\frac{t}{\varkappa}\right) \frac{d \varkappa}{\varkappa}=\frac{1}{k^{\sigma}} B_{k}^{\prime}\left[\frac{1}{t^{\sigma}} \omega(t)\right],
\end{aligned}
$$

где семейство операторов $B_{k}{ }^{\prime}$ связанно с семейством последовательностей $\lambda^{\sigma} \varphi_{\lambda}(t)$ (см. (3.16)). По следствию 2 к лемме 2 получаем

$$
\mathscr{P}_{d, M_{1}+M}^{a, M_{1}+M}\left[B_{k} \omega\right] \leqslant \frac{\mathrm{const}}{k^{\sigma}} \cdot{ }^{I} \mathscr{P}_{(d, M)}^{M_{1}(a, M)}\left[\lambda^{\sigma} \varphi_{\lambda}\right] \cdot{ }_{I} \mathscr{P}_{d, M_{1}}^{a, M_{1}}\left[\frac{1}{t^{\sigma}} \omega(t)\right] .
$$

Осталось заметить, что по лемме П3

$$
{ }_{I} \mathscr{P}_{d, M_{1}}^{a, M_{1}}\left[\left(\frac{1}{t^{\sigma}}\right) \omega(t)\right] \leqslant \text { const } \cdot_{I} \mathscr{P}_{d-\sigma, M_{1}}^{a, M_{1}}[\omega(t)] .
$$

Лемма доказана. 
ЛЕмма П5. Пусть $g \in\left(\mathscr{S}_{b}^{a}\right)^{\prime}, \quad b<a$ (не челье $) ;$ тогда для любого $\varepsilon>0$ существует мера $d \mu(z)$ медленного роста,

$\operatorname{supp} d \mu(z) \subset\{z=x+i y \in \mathbb{C}:|x|<\infty, y=a+\varepsilon\} \cup\{z \in \mathbb{C}:|x|<\infty, y=b-\varepsilon\}$, и числа $c_{j}, \quad j=0,1, \ldots,\langle a\rangle$, такие, что для любой $\varphi(t) \in \mathscr{S}_{b-\varepsilon}^{a+\varepsilon}$ справедливо представление

$$
(g(t), \varphi(t))=\int \widehat{\varphi}(z) d \mu(z)+\sum_{j=0}^{\langle a\rangle} c_{j} \varphi^{(j)}(0) .
$$

ЗАмЕчАниЕ. Если обобщенная функция $g(t)$ квазиасимптотически ограничена относительно $k^{\gamma}$, где $\gamma<-\langle a\rangle-1$, то все $c_{j}$ равны нулю.

ДокАЗАТЕльСтво. Так как $\mathscr{S}_{b}^{a}=\stackrel{0}{\mathscr{S}}_{b}^{a} \oplus\left\{1, t, \ldots, t^{\langle a\rangle}\right\}$, то $g(t)={ }_{g}^{0}(t)+$ $\sum_{j=0}^{\langle a\rangle} c_{j} \delta^{(j)}(t)$, где $\stackrel{0}{g}(t)$ - сужение $g$ на $\stackrel{0}{\mathscr{S}}_{b}^{a}$. Учитывая $(2.15)$, нетрудно убедиться в том, что имеют место следуюшие непрерывные отображения:

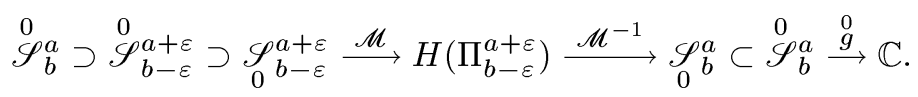

Поэтому определен функционал $\widehat{g}: H\left(\Pi_{b-\varepsilon}^{a+\varepsilon}\right) \mapsto \mathbb{C}$ такой, что справедлива формула

$$
(\stackrel{0}{g}, \varphi)=(\widehat{g}, \mathscr{M} \varphi)=(\widehat{g}, \widehat{\varphi}) \quad \forall \varphi \in \mathscr{S}_{b-\varepsilon}^{a+\varepsilon}
$$

Функционал $\widehat{g}$ определен на функциях, аналитических в полосе $\Pi_{b-\varepsilon}^{a+\varepsilon}$, непрерывных вплоть до границы и убывающих быстрее любой степени на бесконечности. Он может быть продолжен (пользуясь формулой Коши, а затем по теореме Банаха) до функционала над функциями, непрерывными на двух прямых $\{z \in \mathbb{C}:|x|<\infty$, $y=b-\varepsilon\}$ и $\{z \in \mathbb{C}:|x|<\infty, y=a+\varepsilon\}$ и убываюшими на бесконечности быстрее любой степени $|x|^{-1}$. По теореме Рисса он представляется мерой медленного роста, сосредоточенной на этих двух прямых. Лемма доказана.

ЛЕмма П6. Рассмотрим в классе обобщенных функций $\mathscr{S}_{+}^{\prime}$ уравнение

$$
P\left(t \frac{d}{d t}\right) f=g, \quad g \in \mathscr{S}_{+}^{\prime},
$$

где $P(-i z)$ - многочлен степени $n u z_{q}, q=1,2, \ldots, n,-$ его корни. Если $g$ обладает квазиасимптотикой относительно автомодельной функции $\rho(k)$ порядка $\alpha$ и

$$
\operatorname{Im} z_{q} \neq \alpha \quad \text { для } q=1, \ldots, n,
$$

то найдется $f \in \mathscr{S}_{+}^{\prime}-$ решение уравнения (П.41), обладающее квазиасимптотикой относительно $\rho(k)$. 
ДокАЗАТЕЛЬСТво. Поскольку $g$ обладает квазиасимптотикой (а значит, и квазиасимптотически ограничена) относительно $\rho(k)$ порядка автомодельности $\alpha$, то согласно утверждению $2 g \in\left(\mathscr{S}_{b}^{\alpha+\varepsilon}\right)^{\prime}$ с любым $\varepsilon>0$ и некоторым $b$. Выберем $\varepsilon$ настолько малым, чтобы в полосе $\bar{\Pi}_{\alpha-2 \varepsilon}^{\alpha+2 \varepsilon}$ не было нулей полинома $P(-i z)$, что возможно в силу (П.42).

Теперь по лемме П5 и замечанию к ней сушествует мера $d \mu(z)$, сосредоточенная на двух прямых $\{z \in \mathbb{C}:|x|<\infty, y=\alpha+2 \varepsilon\}$ и $\{z \in \mathbb{C}:|x|<\infty, y=b-\varepsilon\}$, такая, что $(g, \varphi)=\int \widehat{\varphi}(z) d \mu(z)$. Определим $f(t)$ формулой

$$
(f(t), \varphi(t))=\int \frac{\widehat{\varphi}(z)}{P(-i z)} d \mu(z), \quad \varphi(t) \in \mathscr{S}_{b-\varepsilon}^{\alpha+2 \varepsilon} .
$$

Ясно, что $f(t) \in\left(\mathscr{S}_{b-\varepsilon}^{\alpha+2 \varepsilon}\right)^{\prime}$. Покажем, что $f(t)$ является решением уравнения (П.41).

Действительно,

$$
\begin{aligned}
\left(P\left(t \frac{d}{d t}\right) f, \varphi\right) & =\left(f, P\left(-\frac{d}{d t} t\right) \varphi\right)=\int \frac{P(-i z) \widehat{\varphi}(z)}{P(-i z)} d \mu(z) \\
& =\int \widehat{\varphi}(z) d \mu(z)=(g, \varphi) .
\end{aligned}
$$

Поскольку в полосе $\bar{\Pi}_{\alpha-2 \varepsilon}^{\alpha+2 \varepsilon}$ нет нулей полинома $P(-i z)$, то по теореме 3 работы [1] это решение обладает квазиасимптотикой относительно $\rho(k)$. Лемма доказана.

\section{Список литературы}

1. Дрожжинов Ю. Н., Завьялов Б. И. Тауберова теорема типа Винера для обобщенных функций медленного роста // Матем. сб. 1998. Т. 189. №7. С. 91-130.

2. Гельфанд И. М., Шилов Г. Е. Обобщенные функции и действия над ними. Вып. 1. М.: Физматгиз, 1959.

3. Владимиров В.С., Дрожжинов Ю.Н., Завьялов Б.И. Многомерные тауберовы теоремы для обобщенных функций. М.: Наука, 1986.

4. Сенета Е. Правильно меняющиеся функции. М.: Наука, 1985.

5. Wiener N. Tauberian theorems // Ann. of Math. 1932. V. 33. № 1. P. 1-100.

6. Винер Н. Интеграл Фурье и некоторые его применения. М.: Физматгиз, 1963.

7. Karamata J. Neuer Beweis und Verallgemeinerung der Tauberschen Sätze, welche die Laplacesche und Stieltjesche Transformation betreffen // Journ. Reine und Angew. Math. 1931. V. 164. № 1. P. 27-29.

8. Beurling A. Sur les inte'grales de Tourier absolument convergentas // Helsinki Comp. Rend. Gie'me Congr. Math. Scand. 1938.

9. Ganelius T. Tauberian remeinder theorems. Berlin-Heidelberg-N. Y.: Springer-Verlag, 1971.

10. Коренблюм Б.И. Обобщение тауберовой теоремы Винера и гармонический анализ быстрорастущих функций // Тр. Моск. матем. об-ва. 1958. Т. 7. С. 121-148.

11. Комеч А. И. Уравнения с однородными ядрами и преобразование Меллина обобщенных функций // ТМФ. 1976. Т. 27. №2. С. 149-162.

12. Земанян А. Г. Интегральные преобразования обобщенных функций. М.: Наука, 1974.

13. Владимиров В. С. Обобщенные функции в математической физике. М.: Наука, 1979.

14. Pilipović S., Stanković B. Wiener Tauberian Theorems for Distribution // J. London Math. Soc. 1993. V. 47. № 2. P. 507-515.

15. Мазья В. Г. Пространства С. Л. Соболева. Л.: Изд-во ЛГУ, 1985. 
16. Брычков Ю. А. Преобразования Меллина. І. М.: ВЦ РАН, 1997.

17. Tröger $G$. A Tauberian Theorem for the Generalized $S_{2}$-Transform // Math. Nachr. 1989. V. 143. P. 55-67.

18. Boas R.P., Widder D. V. The Iterated Stieltjes Transform // Trans. Amer. Math. Soc. 1939. V. 45. P. 1-72.

19. Pilipovič S., Stankovič B., Takači A. Asymptotic Behaviour and Stieltjes Transformation of Distributions. B. 116. Leipzig: Teubner Verlag, 1990.

20. Гарнет Джк. Ограниченные аналитические функции. М.: Мир, 1984.

21. Дрожжинов Ю.Н., Завьялов Б. И. Теоремы типа Винера для обобщенных функций и интегральное преобразование Стилтьеса // Докл. РАН. 1998. Т. 362. № 2. С. 156-159.

22. Иосида К. Функциональный анализ. М.: Мир, 1967.

Математический институт им. В. А. Стеклова РАН, Москва

E-mail: drozzin@genesis.mi.ras.ru
Поступило в редакцию 24.VI.1999 